\title{
Pyrrolide Supported Lanthanide Alkyl Complexes. Influence of Ligands on Molecular Structure and Catalytic Activity toward Isoprene Polymerization
}

Yi Yang, ${ }^{1,2}$ Bo Liu, ${ }^{1,2}$ Kui Lv, ${ }^{1,2}$ Wei Gao, ${ }^{1}$ Dongmei Cui, ${ }^{* 1}$ Xuesi Chen, ${ }^{1}$ Xiabin

$$
\text { Jing }^{1}
$$

${ }^{1}$ State Key Laboratory of Polymer Physics and Chemistry, Changchun Institute of Applied Chemistry, Chinese Academy of Sciences, Changchun 130022, China.

Fax: (+86) 431 5262773; Tel:+86431 5262773; E-mail:dmcui@ciac.jl.cn

${ }^{2}$ Graduate School of the Chinese Academy of Sciences, Beijing 100039, China.

Legends

Stable 1. Summary of crystallographic data for $1 \mathbf{1 a}, \mathbf{1 b}, \mathbf{2 a}, \mathbf{3 a}$ and $\mathbf{4}$

Stables 2-21. Full bond distances and bond angles as well as anisotropic thermal parameters for complexes 1a, 1b, 2a, 3a and 4 . 
STable 1. Summary of crystallographic data for 1a, 1b, 2a, 3a and 4

\begin{tabular}{|c|c|c|c|c|c|}
\hline & $1 \mathrm{a}$ & $1 \mathbf{b}$ & $2 a$ & $3 \mathbf{a}$ & $4-\mathrm{C}_{7} \mathrm{H}_{8}$ \\
\hline formula & $\mathrm{C}_{29} \mathrm{H}_{51} \mathrm{~N}_{2} \mathrm{O}_{2} \mathrm{Si}_{2} \mathrm{Lu}$ & $\mathrm{C}_{25} \mathrm{H}_{43} \mathrm{~N}_{2} \mathrm{OSi}_{2} \mathrm{Sc}$ & $\mathrm{C}_{42} \mathrm{H}_{59} \mathrm{~N}_{4} \mathrm{OSiLu}$ & $\mathrm{C}_{30} \mathrm{H}_{66} \mathrm{~N}_{4} \mathrm{Si}_{4} \mathrm{Y}_{2}$ & $\mathrm{C}_{45} \mathrm{H}_{66} \mathrm{~N}_{8} \mathrm{Si}_{2} \mathrm{Lu}_{2}$ \\
\hline crystal size, $\mathrm{mm}$ & $0.18 \times 0.15 \times 0.13$ & $0.25 \times 0.18 \times 0.16$ & $0.18 \times 0.13 \times 0.11$ & $0.18 \times 0.15 \times 0.13$ & $0.13 \times 0.09 \times 0.07$ \\
\hline formula weight & 690.87 & 488.75 & 838.99 & 773.05 & 1125.18 \\
\hline cryst syst & Monoclinic & Monoclinic & Triclinic & Triclinic & Tetragonal \\
\hline space group & $\mathrm{P} 22_{1} / \mathrm{c}$ & $\mathrm{P} 2(1) / \mathrm{n}$ & $\mathrm{P}-1$ & $\mathrm{P}-1$ & $\mathrm{P} 4(3) 2(1) 2$ \\
\hline$a(\AA)$ & $9.4569(6)$ & $11.5805(8)$ & $10.3754(6)$ & $9.4442(8)$ & $14.9571(7)$ \\
\hline$b(\AA)$ & $16.4263(10)$ & $14.0152(10)$ & $19.2503(11)$ & $10.4910(9)$ & $14.9571(7)$ \\
\hline$c(\AA)$ & $21.2020(13)$ & $17.7458(13)$ & $20.9445(12)$ & $11.4717(10)$ & $21.8098(14)$ \\
\hline$\alpha(\operatorname{deg})$ & 90 & 90 & $88.3420(10)$ & $77.9230(10)$ & 90 \\
\hline$\beta(\operatorname{deg})$ & $92.3310(10)$ & $90.5080(10)$ & $89.6590(10)$ & $84.3380(10)$ & 90 \\
\hline$\gamma(\operatorname{deg})$ & 90 & 90 & $88.0490(10)$ & $65.7770(10)$ & 90 \\
\hline$V\left(\AA^{3}\right)$ & $3290.8(4)$ & $2880.1(4)$ & $4179.0(4)$ & $1013.49(15)$ & $4879.2(4)$ \\
\hline$Z$ & 4 & 4 & 4 & 1 & 4 \\
\hline$D_{\text {calcd }}\left(\mathrm{g} / \mathrm{cm}^{3}\right)$ & 1.394 & 1.127 & 1.334 & 1.267 & 1.532 \\
\hline radiation $(\lambda), \AA$ & $\operatorname{MoK} \alpha(0.71073)$ & $\operatorname{MoK} \alpha(0.71073)$ & Mo K $\alpha(0.71073)$ & Mo K $\alpha(0.71073)$ & Mo K $\alpha(0.71073)$ \\
\hline $2 \theta_{\max }, \operatorname{deg}$ & 52.06 & 52.08 & 52.00 & 52.08 & 52.08 \\
\hline$\mu\left(\mathrm{cm}^{-1}\right)$ & 30.98 & 3.56 & 24.26 & 29.90 & 41.10 \\
\hline $\mathrm{F}(000)$ & 1416 & 1056 & 1728 & 408 & 2248 \\
\hline no. of obsd reflns & 6444 & 5680 & 16002 & 3900 & 4826 \\
\hline $\begin{array}{l}\text { no. of params } \\
\text { refnd }\end{array}$ & 352 & 288 & 905 & 189 & 266 \\
\hline GOF & 0.928 & 1.021 & 0.870 & 0.996 & 0.965 \\
\hline$R 1$ & 0.0252 & 0.0416 & 0.0405 & 0.0389 & 0.0184 \\
\hline $\mathrm{w} R 2$ & 0.0515 & 0.1114 & 0.0860 & 0.0987 & 0.0411 \\
\hline
\end{tabular}


Stable 2. Crystal data and structure refinement for $1 \mathrm{a}$

Identification code

Empirical formula

Formula weight

Temperature

Wavelength

Crystal system

Space group

Unit cell dimensions

Volume

Z

Density (calculated)

Absorption coefficient

$\mathrm{F}(000)$

Crystal size

Theta range for data collection

Index ranges

Reflections collected

Independent reflections

Completeness to theta $=26.03^{\circ}$

Absorption correction

Max. and min. transmission

Refinement method

Data / restraints / parameters q262-2

C29 H51 Lu N2 O2 Si2

690.87

187(2) K

$0.71073 \AA$

Monoclinic

$\mathrm{P} 2{ }_{1} / \mathrm{c}$

$a=9.4569(6) \AA$

$\alpha=90^{\circ}$.

$\mathrm{b}=16.4263(10) \AA$

$\beta=92.3310(10)^{\circ}$.

$\mathrm{c}=21.2020(13) \AA$

$\gamma=90^{\circ}$.

$3290.8(4) \AA^{3}$

4

$1.394 \mathrm{Mg} / \mathrm{m}^{3}$

$3.098 \mathrm{~mm}^{-1}$

1416

$0.18 \times 0.15 \times 0.13 \mathrm{~mm}^{3}$

1.57 to $26.03^{\circ}$.

$-11<=\mathrm{h}<=8,-20<=\mathrm{k}<=20,-23<=\mathrm{l}<=26$

18213

$6444[\mathrm{R}($ int $)=0.0461]$

$99.4 \%$

Semi-empirical from equivalents

0.6888 and 0.6055

Full-matrix least-squares on $\mathrm{F}^{2}$

$6444 / 71 / 352$ 
Goodness-of-fit on $\mathrm{F}^{2}$

Final $\mathrm{R}$ indices [I $>2 \operatorname{sigma}(\mathrm{I})]$

$\mathrm{R}$ indices (all data)

Largest diff. peak and hole
0.928

$\mathrm{R} 1=0.0252, \mathrm{wR} 2=0.0496$

$\mathrm{R} 1=0.0346, \mathrm{wR} 2=0.0515$

0.973 and $-0.460 \mathrm{e}^{-3}$ 
Stable 3. Atomic coordinates $\left(\begin{array}{ll}x & 1^{4}\end{array}\right)$ and equivalent isotropic displacement parameters $\left(\AA^{2} \times 10^{3}\right)$ for $1 \mathrm{a}$. $U(\mathrm{eq})$ is defined as one third of the trace of the orthogonalized Uij tensor.

\begin{tabular}{|c|c|c|c|c|}
\hline & $\mathrm{x}$ & $\mathrm{y}$ & z & $\mathrm{U}(\mathrm{eq})$ \\
\hline $\mathrm{Lu}$ & 2161(1) & $7695(1)$ & $4143(1)$ & $27(1)$ \\
\hline $\mathrm{N}(1)$ & $1315(3)$ & $7403(2)$ & $3014(1)$ & $30(1)$ \\
\hline $\mathrm{N}(2)$ & $3489(3)$ & $8428(2)$ & $3423(1)$ & $34(1)$ \\
\hline $\mathrm{O}(1)$ & $3884(2)$ & $6708(1)$ & $4050(1)$ & $38(1)$ \\
\hline $\mathrm{O}(2)$ & $527(2)$ & $8750(1)$ & $4040(1)$ & $33(1)$ \\
\hline $\operatorname{Si}(1)$ & $4522(1)$ & 8311(1) & $5647(1)$ & $35(1)$ \\
\hline $\operatorname{Si}(2)$ & $18(1)$ & $6579(1)$ & $5346(1)$ & $34(1)$ \\
\hline $\mathrm{C}(1)$ & $110(3)$ & $6942(2)$ & $2779(1)$ & $33(1)$ \\
\hline$C(2)$ & $180(4)$ & $6087(2)$ & 2794(1) & $41(1)$ \\
\hline$C(3)$ & $-1015(5)$ & $5650(2)$ & $2601(2)$ & $54(1)$ \\
\hline$C(4)$ & $-2226(5)$ & $6030(3)$ & $2385(2)$ & $57(1)$ \\
\hline$C(5)$ & $-2283(4)$ & $6866(3)$ & $2368(2)$ & $52(1)$ \\
\hline$C(6)$ & $-1123(4)$ & $7337(2)$ & $2567(2)$ & $38(1)$ \\
\hline$C(7)$ & $1525(4)$ & $5664(2)$ & $2984(2)$ & $55(1)$ \\
\hline$C(8)$ & $-1212(4)$ & $8257(2)$ & $2529(2)$ & $53(1)$ \\
\hline$C(9)$ & $2008(3)$ & $7812(2)$ & 2611(1) & $31(1)$ \\
\hline$C(10)$ & $3147(3)$ & $8328(2)$ & $2790(1)$ & $33(1)$ \\
\hline $\mathrm{C}(11)$ & $4044(4)$ & $8797(2)$ & $2430(2)$ & $42(1)$ \\
\hline$C(12)$ & $4951(4)$ & $9193(2)$ & $2854(2)$ & $43(1)$ \\
\hline$C(13)$ & $4593(3)$ & $8953(2)$ & $3447(2)$ & $39(1)$ \\
\hline
\end{tabular}




\begin{tabular}{|c|c|c|c|c|}
\hline$C(14)$ & $3081(3)$ & $8388(2)$ & $5040(1)$ & $40(1)$ \\
\hline$C(15)$ & $5327(4)$ & $7269(2)$ & $5735(2)$ & $54(1)$ \\
\hline$C(16)$ & $6019(4)$ & $9006(2)$ & $5458(2)$ & $62(1)$ \\
\hline$C(17)$ & $3925(4)$ & $8596(2)$ & $6451(2)$ & $58(1)$ \\
\hline $\mathrm{C}(18)$ & $420(3)$ & $6767(2)$ & $4517(1)$ & $32(1)$ \\
\hline$C(19)$ & $-1451(4)$ & $5829(2)$ & $5459(2)$ & $54(1)$ \\
\hline$C(20)$ & $-519(4)$ & $7542(2)$ & $5755(2)$ & $50(1)$ \\
\hline $\mathrm{C}(21)$ & $1560(4)$ & $6148(2)$ & $5813(2)$ & $56(1)$ \\
\hline$C(22)$ & $939(4)$ & $9576(2)$ & $3883(2)$ & $53(1)$ \\
\hline$C(23)$ & $-323(5)$ & $10093(2)$ & $3935(2)$ & $77(1)$ \\
\hline$C(24)$ & $-1493(4)$ & $9581(2)$ & $4060(2)$ & $70(1)$ \\
\hline$C(25)$ & $-916(4)$ & $8766(2)$ & $4242(2)$ & $58(1)$ \\
\hline$C(26)$ & $5122(4)$ & $6750(3)$ & $3663(2)$ & $83(2)$ \\
\hline C(29) & $3948(4)$ & $5950(2)$ & $4406(2)$ & $45(1)$ \\
\hline $\mathrm{C}(27 \mathrm{~A})$ & $5610(20)$ & $5836(11)$ & $3620(8)$ & $59(4)$ \\
\hline $\mathrm{C}(27 \mathrm{~B})$ & $6112(11)$ & $6121(8)$ & $3895(7)$ & $82(3)$ \\
\hline $\mathrm{C}(28 \mathrm{~A})$ & $5160(40)$ & $5470(20)$ & $4260(11)$ & $71(6)$ \\
\hline $\mathrm{C}(28 \mathrm{~B})$ & $4995(18)$ & $5443(10)$ & $4042(7)$ & $74(4)$ \\
\hline
\end{tabular}


STable 4. Anisotropic displacement parameters $\left(\AA^{2} x 1^{3}\right)$ for 1 a. The anisotropic displacement factor exponent takes the form: $-2 \pi^{2}\left[h^{2} a * 2 U^{11}+\ldots\right.$ $\left.+2 \mathrm{~h} \mathrm{k} \mathrm{a*} \mathbf{b}^{*} \mathbf{U}^{12}\right]$

\begin{tabular}{|c|c|c|c|c|c|c|}
\hline & U11 & $\mathrm{U} 22$ & U33 & $\mathrm{U} 23$ & U13 & U12 \\
\hline $\mathrm{Lu}$ & $28(1)$ & $26(1)$ & $27(1)$ & 1(1) & 1(1) & $0(1)$ \\
\hline $\mathrm{N}(1)$ & $35(2)$ & $27(2)$ & $28(1)$ & $-2(1)$ & $3(1)$ & $-1(1)$ \\
\hline $\mathrm{N}(2)$ & $37(2)$ & $35(2)$ & $29(2)$ & $1(1)$ & $3(1)$ & $-3(1)$ \\
\hline $\mathrm{O}(1)$ & $34(1)$ & $36(1)$ & $44(1)$ & $6(1)$ & $10(1)$ & $9(1)$ \\
\hline $\mathrm{O}(2)$ & $33(1)$ & $25(1)$ & $41(1)$ & $5(1)$ & $-1(1)$ & $2(1)$ \\
\hline $\mathrm{Si}(1)$ & $31(1)$ & $34(1)$ & $39(1)$ & $-1(1)$ & $-3(1)$ & $-2(1)$ \\
\hline $\operatorname{Si}(2)$ & $36(1)$ & $30(1)$ & $38(1)$ & $2(1)$ & $7(1)$ & $-2(1)$ \\
\hline$C(1)$ & $37(2)$ & $37(2)$ & $24(2)$ & $-6(1)$ & $0(1)$ & $-8(2)$ \\
\hline $\mathrm{C}(2)$ & $59(3)$ & $38(2)$ & $25(2)$ & $-4(2)$ & $3(2)$ & $-7(2)$ \\
\hline$C(3)$ & $84(3)$ & $44(2)$ & $34(2)$ & $-6(2)$ & $0(2)$ & $-26(2)$ \\
\hline $\mathrm{C}(4)$ & $64(3)$ & $69(3)$ & $39(2)$ & $-9(2)$ & $1(2)$ & $-36(2)$ \\
\hline $\mathrm{C}(5)$ & $42(2)$ & $74(3)$ & $38(2)$ & $-4(2)$ & $0(2)$ & $-14(2)$ \\
\hline$C(6)$ & $40(2)$ & $47(2)$ & $26(2)$ & $-6(2)$ & $1(2)$ & $-5(2)$ \\
\hline$C(7)$ & $87(3)$ & $31(2)$ & $46(2)$ & $-6(2)$ & $-9(2)$ & $4(2)$ \\
\hline $\mathrm{C}(8)$ & $50(2)$ & $54(3)$ & $54(2)$ & $-11(2)$ & $-7(2)$ & $7(2)$ \\
\hline C(9) & $37(2)$ & $30(2)$ & $27(2)$ & $-2(1)$ & $0(1)$ & $5(2)$ \\
\hline$C(10)$ & $36(2)$ & $31(2)$ & $31(2)$ & $0(1)$ & $5(2)$ & $3(2)$ \\
\hline $\mathrm{C}(11)$ & $48(2)$ & $45(2)$ & $35(2)$ & $7(2)$ & $9(2)$ & $-6(2)$ \\
\hline$C(12)$ & $40(2)$ & $40(2)$ & $50(2)$ & $6(2)$ & $15(2)$ & $-9(2)$ \\
\hline$C(13)$ & $33(2)$ & $42(2)$ & $43(2)$ & $-2(2)$ & $1(2)$ & $-10(2)$ \\
\hline
\end{tabular}




\begin{tabular}{|c|c|c|c|c|c|c|}
\hline$C(14)$ & $38(2)$ & $41(2)$ & $39(2)$ & $-5(2)$ & $-3(2)$ & $1(2)$ \\
\hline$C(15)$ & $51(2)$ & 41(2) & 70(3) & $-3(2)$ & $-11(2)$ & $9(2)$ \\
\hline$C(16)$ & $40(2)$ & $55(3)$ & 91(3) & $13(2)$ & $-1(2)$ & $-8(2)$ \\
\hline$C(17)$ & $57(3)$ & $76(3)$ & $40(2)$ & $0(2)$ & $-4(2)$ & $16(2)$ \\
\hline$C(18)$ & $33(2)$ & $24(2)$ & $40(2)$ & $0(1)$ & $4(2)$ & $0(1)$ \\
\hline$C(19)$ & 51(3) & $44(2)$ & $69(3)$ & $6(2)$ & $15(2)$ & $-7(2)$ \\
\hline$C(20)$ & $68(3)$ & $39(2)$ & $46(2)$ & $-3(2)$ & $18(2)$ & $-2(2)$ \\
\hline$C(21)$ & $53(3)$ & $74(3)$ & $42(2)$ & $10(2)$ & 1(2) & $8(2)$ \\
\hline$C(22)$ & $50(2)$ & $28(2)$ & $80(3)$ & $14(2)$ & $-5(2)$ & $-5(2)$ \\
\hline$C(23)$ & $82(4)$ & 41(3) & 111(4) & 20(3) & $33(3)$ & $19(2)$ \\
\hline$C(24)$ & 49(3) & $46(3)$ & $115(4)$ & 2(3) & $5(3)$ & $15(2)$ \\
\hline$C(25)$ & $44(2)$ & $49(2)$ & $82(3)$ & $18(2)$ & $23(2)$ & $13(2)$ \\
\hline$C(26)$ & $59(3)$ & $80(3)$ & 113(4) & $34(3)$ & $50(3)$ & $29(3)$ \\
\hline$C(29)$ & $52(2)$ & $35(2)$ & $46(2)$ & $4(2)$ & $3(2)$ & $8(2)$ \\
\hline$C(27 \mathrm{~A})$ & $61(10)$ & $63(8)$ & $54(8)$ & $8(7)$ & $14(7)$ & $28(7)$ \\
\hline $\mathrm{C}(27 \mathrm{~B})$ & $68(6)$ & $116(8)$ & 63(7) & $32(6)$ & $28(5)$ & $49(5)$ \\
\hline$C(28 \mathrm{~A})$ & $75(10)$ & $79(10)$ & $61(13)$ & $26(10)$ & $25(10)$ & $40(8)$ \\
\hline$C(28 B)$ & 101(7) & $67(5)$ & $55(8)$ & $22(6)$ & 19(7) & $57(4)$ \\
\hline
\end{tabular}


Stable 5. Bond lengths $[\AA ̊]$ and angles $\left[^{\circ}\right]$ for $1 a$.

\begin{tabular}{|c|c|c|c|}
\hline $\mathrm{Lu}-\mathrm{O}(1)$ & $2.313(2)$ & $C(2)-C(7)$ & $1.491(5)$ \\
\hline $\mathrm{Lu}-\mathrm{O}(2)$ & $2.326(2)$ & $C(3)-C(4)$ & $1.367(5)$ \\
\hline $\mathrm{Lu}-\mathrm{N}(2)$ & $2.349(3)$ & $\mathrm{C}(3)-\mathrm{H}(3)$ & 0.9300 \\
\hline $\mathrm{Lu}-\mathrm{C}(14)$ & $2.351(3)$ & $C(4)-C(5)$ & $1.376(5)$ \\
\hline Lu-C(18) & $2.401(3)$ & $\mathrm{C}(4)-\mathrm{H}(4)$ & 0.9300 \\
\hline Lu-N(1) & $2.540(2)$ & $C(5)-C(6)$ & $1.394(5)$ \\
\hline $\mathrm{N}(1)-\mathrm{C}(9)$ & $1.287(4)$ & $\mathrm{C}(5)-\mathrm{H}(5)$ & 0.9300 \\
\hline $\mathrm{N}(1)-\mathrm{C}(1)$ & $1.439(4)$ & $C(6)-C(8)$ & $1.516(5)$ \\
\hline$N(2)-C(13)$ & $1.354(4)$ & $\mathrm{C}(7)-\mathrm{H}(7 \mathrm{~A})$ & 0.9600 \\
\hline $\mathrm{N}(2)-\mathrm{C}(10)$ & $1.378(4)$ & $\mathrm{C}(7)-\mathrm{H}(7 \mathrm{~B})$ & 0.9600 \\
\hline $\mathrm{O}(1)-\mathrm{C}(29)$ & $1.456(4)$ & $\mathrm{C}(7)-\mathrm{H}(7 \mathrm{C})$ & 0.9600 \\
\hline $\mathrm{O}(1)-\mathrm{C}(26)$ & $1.458(4)$ & $\mathrm{C}(8)-\mathrm{H}(8 \mathrm{~A})$ & 0.9600 \\
\hline $\mathrm{O}(2)-\mathrm{C}(25)$ & $1.446(4)$ & $\mathrm{C}(8)-\mathrm{H}(8 \mathrm{~B})$ & 0.9600 \\
\hline $\mathrm{O}(2)-\mathrm{C}(22)$ & $1.454(4)$ & $\mathrm{C}(8)-\mathrm{H}(8 \mathrm{C})$ & 0.9600 \\
\hline $\mathrm{Si}(1)-\mathrm{C}(14)$ & $1.840(3)$ & $C(9)-C(10)$ & $1.411(4)$ \\
\hline $\mathrm{Si}(1)-\mathrm{C}(16)$ & $1.876(4)$ & $\mathrm{C}(9)-\mathrm{H}(9)$ & 0.9300 \\
\hline $\mathrm{Si}(1)-\mathrm{C}(17)$ & $1.877(3)$ & $\mathrm{C}(10)-\mathrm{C}(11)$ & $1.396(4)$ \\
\hline $\mathrm{Si}(1)-\mathrm{C}(15)$ & $1.880(3)$ & $\mathrm{C}(11)-\mathrm{C}(12)$ & $1.380(4)$ \\
\hline $\mathrm{Si}(2)-\mathrm{C}(18)$ & $1.841(3)$ & $\mathrm{C}(11)-\mathrm{H}(11)$ & 0.9300 \\
\hline $\mathrm{Si}(2)-\mathrm{C}(21)$ & $1.868(3)$ & $\mathrm{C}(12)-\mathrm{C}(13)$ & $1.374(4)$ \\
\hline $\mathrm{Si}(2)-\mathrm{C}(19)$ & $1.880(3)$ & $\mathrm{C}(12)-\mathrm{H}(12)$ & 0.9300 \\
\hline $\mathrm{Si}(2)-\mathrm{C}(20)$ & $1.883(3)$ & $\mathrm{C}(13)-\mathrm{H}(13)$ & 0.9300 \\
\hline$C(1)-C(6)$ & $1.393(4)$ & $\mathrm{C}(14)-\mathrm{H}(14 \mathrm{~A})$ & 0.9700 \\
\hline$C(1)-C(2)$ & $1.407(4)$ & $\mathrm{C}(14)-\mathrm{H}(14 \mathrm{~B})$ & 0.9700 \\
\hline$C(2)-C(3)$ & $1.387(5)$ & $\mathrm{C}(15)-\mathrm{H}(15 \mathrm{~A})$ & 0.9600 \\
\hline
\end{tabular}




\begin{tabular}{|c|c|c|c|}
\hline $\mathrm{C}(15)-\mathrm{H}(15 \mathrm{~B})$ & 0.9600 & $\mathrm{C}(24)-\mathrm{H}(24 \mathrm{~A})$ & 0.9700 \\
\hline $\mathrm{C}(15)-\mathrm{H}(15 \mathrm{C})$ & 0.9600 & $\mathrm{C}(24)-\mathrm{H}(24 \mathrm{~B})$ & 0.9700 \\
\hline$C(16)-H(16 A)$ & 0.9600 & $\mathrm{C}(25)-\mathrm{H}(25 \mathrm{~A})$ & 0.9700 \\
\hline $\mathrm{C}(16)-\mathrm{H}(16 \mathrm{~B})$ & 0.9600 & $\mathrm{C}(25)-\mathrm{H}(25 \mathrm{~B})$ & 0.9700 \\
\hline$C(16)-H(16 C)$ & 0.9600 & $\mathrm{C}(26)-\mathrm{C}(27 \mathrm{~B})$ & $1.466(9)$ \\
\hline $\mathrm{C}(17)-\mathrm{H}(17 \mathrm{~A})$ & 0.9600 & $C(26)-C(27 A)$ & $1.575(16)$ \\
\hline $\mathrm{C}(17)-\mathrm{H}(17 \mathrm{~B})$ & 0.9600 & $\mathrm{C}(26)-\mathrm{H}(26 \mathrm{~A})$ & 0.9700 \\
\hline $\mathrm{C}(17)-\mathrm{H}(17 \mathrm{C})$ & 0.9600 & $\mathrm{C}(26)-\mathrm{H}(26 \mathrm{~B})$ & 0.9700 \\
\hline $\mathrm{C}(18)-\mathrm{H}(18 \mathrm{~A})$ & 0.9700 & $\mathrm{C}(26)-\mathrm{H}(26 \mathrm{C})$ & 0.9700 \\
\hline $\mathrm{C}(18)-\mathrm{H}(18 \mathrm{~B})$ & 0.9700 & $\mathrm{C}(26)-\mathrm{H}(26 \mathrm{D})$ & 0.9700 \\
\hline $\mathrm{C}(19)-\mathrm{H}(19 \mathrm{~A})$ & 0.9600 & $C(29)-C(28 A)$ & $1.43(3)$ \\
\hline $\mathrm{C}(19)-\mathrm{H}(19 \mathrm{~B})$ & 0.9600 & $\mathrm{C}(29)-\mathrm{C}(28 \mathrm{~B})$ & $1.527(19)$ \\
\hline $\mathrm{C}(19)-\mathrm{H}(19 \mathrm{C})$ & 0.9600 & $\mathrm{C}(29)-\mathrm{H}(29 \mathrm{~A})$ & 0.9700 \\
\hline $\mathrm{C}(20)-\mathrm{H}(20 \mathrm{~A})$ & 0.9600 & $\mathrm{C}(29)-\mathrm{H}(29 \mathrm{~B})$ & 0.9700 \\
\hline $\mathrm{C}(20)-\mathrm{H}(20 \mathrm{~B})$ & 0.9600 & $\mathrm{C}(29)-\mathrm{H}(29 \mathrm{C})$ & 0.9700 \\
\hline $\mathrm{C}(20)-\mathrm{H}(20 \mathrm{C})$ & 0.9600 & $\mathrm{C}(29)-\mathrm{H}(29 \mathrm{D})$ & 0.9700 \\
\hline $\mathrm{C}(21)-\mathrm{H}(21 \mathrm{~A})$ & 0.9600 & $\mathrm{C}(27 \mathrm{~A})-\mathrm{C}(28 \mathrm{~A})$ & $1.557(15)$ \\
\hline $\mathrm{C}(21)-\mathrm{H}(21 \mathrm{~B})$ & 0.9600 & $\mathrm{C}(27 \mathrm{~A})-\mathrm{H}(27 \mathrm{~A})$ & 0.9700 \\
\hline $\mathrm{C}(21)-\mathrm{H}(21 \mathrm{C})$ & 0.9600 & $\mathrm{C}(27 \mathrm{~A})-\mathrm{H}(27 \mathrm{~B})$ & 0.9700 \\
\hline$C(22)-C(23)$ & $1.473(5)$ & $\mathrm{C}(27 \mathrm{~B})-\mathrm{C}(28 \mathrm{~B})$ & $1.574(13)$ \\
\hline $\mathrm{C}(22)-\mathrm{H}(22 \mathrm{~A})$ & 0.9700 & $\mathrm{C}(27 \mathrm{~B})-\mathrm{H}(27 \mathrm{C})$ & 0.9700 \\
\hline $\mathrm{C}(22)-\mathrm{H}(22 \mathrm{~B})$ & 0.9700 & $\mathrm{C}(27 \mathrm{~B})-\mathrm{H}(27 \mathrm{D})$ & 0.9700 \\
\hline$C(23)-C(24)$ & $1.424(5)$ & $\mathrm{C}(28 \mathrm{~A})-\mathrm{H}(28 \mathrm{~A})$ & 0.9700 \\
\hline $\mathrm{C}(23)-\mathrm{H}(23 \mathrm{~A})$ & 0.9700 & $\mathrm{C}(28 \mathrm{~A})-\mathrm{H}(28 \mathrm{~B})$ & 0.9700 \\
\hline $\mathrm{C}(23)-\mathrm{H}(23 \mathrm{~B})$ & 0.9700 & $\mathrm{C}(28 \mathrm{~B})-\mathrm{H}(28 \mathrm{C})$ & 0.9700 \\
\hline$C(24)-C(25)$ & $1.490(5)$ & $\mathrm{C}(28 \mathrm{~B})-\mathrm{H}(28 \mathrm{D})$ & 0.9700 \\
\hline $\mathrm{O}(1)-\mathrm{Lu}-\mathrm{O}(2)$ & $169.04(7)$ & $\mathrm{O}(1)-\mathrm{Lu}-\mathrm{N}(2)$ & $84.60(8)$ \\
\hline
\end{tabular}




\begin{tabular}{|c|c|c|c|}
\hline $\mathrm{O}(2)-\mathrm{Lu}-\mathrm{N}(2)$ & $85.88(8)$ & $C(16)-S i(1)-C(17)$ & $107.43(18)$ \\
\hline $\mathrm{O}(1)-\mathrm{Lu}-\mathrm{C}(14)$ & $99.72(10)$ & $\mathrm{C}(14)-\mathrm{Si}(1)-\mathrm{C}(15)$ & $114.67(16)$ \\
\hline $\mathrm{O}(2)-\mathrm{Lu}-\mathrm{C}(14)$ & $86.49(9)$ & $C(16)-\operatorname{Si}(1)-C(15)$ & $105.62(18)$ \\
\hline $\mathrm{N}(2)-\mathrm{Lu}-\mathrm{C}(14)$ & $95.04(11)$ & $C(17)-\operatorname{Si}(1)-C(15)$ & $105.82(17)$ \\
\hline $\mathrm{O}(1)-\mathrm{Lu}-\mathrm{C}(18)$ & $94.47(9)$ & $\mathrm{C}(18)-\mathrm{Si}(2)-\mathrm{C}(21)$ & $112.49(15)$ \\
\hline $\mathrm{O}(2)-\mathrm{Lu}-\mathrm{C}(18)$ & $92.43(9)$ & $\mathrm{C}(18)-\mathrm{Si}(2)-\mathrm{C}(19)$ & $114.46(15)$ \\
\hline $\mathrm{N}(2)-\mathrm{Lu}-\mathrm{C}(18)$ & $158.67(9)$ & $\mathrm{C}(21)-\mathrm{Si}(2)-\mathrm{C}(19)$ & $104.37(17)$ \\
\hline $\mathrm{C}(14)-\mathrm{Lu}-\mathrm{C}(18)$ & $106.09(11)$ & $\mathrm{C}(18)-\mathrm{Si}(2)-\mathrm{C}(20)$ & $111.69(15)$ \\
\hline $\mathrm{O}(1)-\mathrm{Lu}-\mathrm{N}(1)$ & $89.02(8)$ & $\mathrm{C}(21)-\mathrm{Si}(2)-\mathrm{C}(20)$ & $107.09(18)$ \\
\hline $\mathrm{O}(2)-\mathrm{Lu}-\mathrm{N}(1)$ & $82.50(8)$ & $C(19)-\operatorname{Si}(2)-C(20)$ & $106.13(17)$ \\
\hline $\mathrm{N}(2)-\mathrm{Lu}-\mathrm{N}(1)$ & $68.87(8)$ & $C(6)-C(1)-C(2)$ & $120.7(3)$ \\
\hline $\mathrm{C}(14)-\mathrm{Lu}-\mathrm{N}(1)$ & $161.03(10)$ & $C(6)-C(1)-N(1)$ & $120.5(3)$ \\
\hline $\mathrm{C}(18)-\mathrm{Lu}-\mathrm{N}(1)$ & $89.82(9)$ & $\mathrm{C}(2)-\mathrm{C}(1)-\mathrm{N}(1)$ & $118.8(3)$ \\
\hline $\mathrm{C}(9)-\mathrm{N}(1)-\mathrm{C}(1)$ & $117.5(3)$ & $C(3)-C(2)-C(1)$ & $118.3(3)$ \\
\hline $\mathrm{C}(9)-\mathrm{N}(1)-\mathrm{Lu}$ & $112.2(2)$ & $C(3)-C(2)-C(7)$ & $121.0(3)$ \\
\hline $\mathrm{C}(1)-\mathrm{N}(1)-\mathrm{Lu}$ & $129.78(18)$ & $C(1)-C(2)-C(7)$ & $120.7(3)$ \\
\hline$C(13)-N(2)-C(10)$ & $105.3(3)$ & $C(4)-C(3)-C(2)$ & $121.6(4)$ \\
\hline$C(13)-N(2)-L u$ & $137.2(2)$ & $\mathrm{C}(4)-\mathrm{C}(3)-\mathrm{H}(3)$ & 119.2 \\
\hline $\mathrm{C}(10)-\mathrm{N}(2)-\mathrm{Lu}$ & $117.6(2)$ & $\mathrm{C}(2)-\mathrm{C}(3)-\mathrm{H}(3)$ & 119.2 \\
\hline $\mathrm{C}(29)-\mathrm{O}(1)-\mathrm{C}(26)$ & $108.4(3)$ & $C(3)-C(4)-C(5)$ & $119.7(4)$ \\
\hline $\mathrm{C}(29)-\mathrm{O}(1)-\mathrm{Lu}$ & 124.74(19) & $\mathrm{C}(3)-\mathrm{C}(4)-\mathrm{H}(4)$ & 120.1 \\
\hline $\mathrm{C}(26)-\mathrm{O}(1)-\mathrm{Lu}$ & $126.8(2)$ & $\mathrm{C}(5)-\mathrm{C}(4)-\mathrm{H}(4)$ & 120.1 \\
\hline $\mathrm{C}(25)-\mathrm{O}(2)-\mathrm{C}(22)$ & $108.4(2)$ & $C(4)-C(5)-C(6)$ & $121.1(4)$ \\
\hline $\mathrm{C}(25)-\mathrm{O}(2)-\mathrm{Lu}$ & $128.08(19)$ & $\mathrm{C}(4)-\mathrm{C}(5)-\mathrm{H}(5)$ & 119.4 \\
\hline $\mathrm{C}(22)-\mathrm{O}(2)-\mathrm{Lu}$ & $122.22(19)$ & $\mathrm{C}(6)-\mathrm{C}(5)-\mathrm{H}(5)$ & 119.4 \\
\hline $\mathrm{C}(14)-\mathrm{Si}(1)-\mathrm{C}(16)$ & $110.66(17)$ & $C(1)-C(6)-C(5)$ & $118.6(3)$ \\
\hline $\mathrm{C}(14)-\mathrm{Si}(1)-\mathrm{C}(17)$ & $112.16(16)$ & $C(1)-C(6)-C(8)$ & $121.7(3)$ \\
\hline
\end{tabular}




\begin{tabular}{|c|c|c|c|}
\hline$C(5)-C(6)-C(8)$ & $119.7(3)$ & $\mathrm{C}(12)-\mathrm{C}(13)-\mathrm{H}(13)$ & 124.3 \\
\hline $\mathrm{C}(2)-\mathrm{C}(7)-\mathrm{H}(7 \mathrm{~A})$ & 109.5 & $\mathrm{Si}(1)-\mathrm{C}(14)-\mathrm{Lu}$ & $140.49(17)$ \\
\hline $\mathrm{C}(2)-\mathrm{C}(7)-\mathrm{H}(7 \mathrm{~B})$ & 109.5 & $\mathrm{Si}(1)-\mathrm{C}(14)-\mathrm{H}(14 \mathrm{~A})$ & 101.9 \\
\hline $\mathrm{H}(7 \mathrm{~A})-\mathrm{C}(7)-\mathrm{H}(7 \mathrm{~B})$ & 109.5 & $\mathrm{Lu}-\mathrm{C}(14)-\mathrm{H}(14 \mathrm{~A})$ & 101.9 \\
\hline $\mathrm{C}(2)-\mathrm{C}(7)-\mathrm{H}(7 \mathrm{C})$ & 109.5 & $\mathrm{Si}(1)-\mathrm{C}(14)-\mathrm{H}(14 \mathrm{~B})$ & 101.9 \\
\hline $\mathrm{H}(7 \mathrm{~A})-\mathrm{C}(7)-\mathrm{H}(7 \mathrm{C})$ & 109.5 & $\mathrm{Lu}-\mathrm{C}(14)-\mathrm{H}(14 \mathrm{~B})$ & 101.9 \\
\hline $\mathrm{H}(7 \mathrm{~B})-\mathrm{C}(7)-\mathrm{H}(7 \mathrm{C})$ & 109.5 & $\mathrm{H}(14 \mathrm{~A})-\mathrm{C}(14)-\mathrm{H}(14 \mathrm{~B})$ & 104.7 \\
\hline $\mathrm{C}(6)-\mathrm{C}(8)-\mathrm{H}(8 \mathrm{~A})$ & 109.5 & $\mathrm{Si}(1)-\mathrm{C}(15)-\mathrm{H}(15 \mathrm{~A})$ & 109.5 \\
\hline $\mathrm{C}(6)-\mathrm{C}(8)-\mathrm{H}(8 \mathrm{~B})$ & 109.5 & $\mathrm{Si}(1)-\mathrm{C}(15)-\mathrm{H}(15 \mathrm{~B})$ & 109.5 \\
\hline $\mathrm{H}(8 \mathrm{~A})-\mathrm{C}(8)-\mathrm{H}(8 \mathrm{~B})$ & 109.5 & $\mathrm{H}(15 \mathrm{~A})-\mathrm{C}(15)-\mathrm{H}(15 \mathrm{~B})$ & 109.5 \\
\hline $\mathrm{C}(6)-\mathrm{C}(8)-\mathrm{H}(8 \mathrm{C})$ & 109.5 & $\mathrm{Si}(1)-\mathrm{C}(15)-\mathrm{H}(15 \mathrm{C})$ & 109.5 \\
\hline $\mathrm{H}(8 \mathrm{~A})-\mathrm{C}(8)-\mathrm{H}(8 \mathrm{C})$ & 109.5 & $\mathrm{H}(15 \mathrm{~A})-\mathrm{C}(15)-\mathrm{H}(15 \mathrm{C})$ & 109.5 \\
\hline $\mathrm{H}(8 \mathrm{~B})-\mathrm{C}(8)-\mathrm{H}(8 \mathrm{C})$ & 109.5 & $\mathrm{H}(15 \mathrm{~B})-\mathrm{C}(15)-\mathrm{H}(15 \mathrm{C})$ & 109.5 \\
\hline $\mathrm{N}(1)-\mathrm{C}(9)-\mathrm{C}(10)$ & $122.6(3)$ & $\mathrm{Si}(1)-\mathrm{C}(16)-\mathrm{H}(16 \mathrm{~A})$ & 109.5 \\
\hline $\mathrm{N}(1)-\mathrm{C}(9)-\mathrm{H}(9)$ & 118.7 & $\mathrm{Si}(1)-\mathrm{C}(16)-\mathrm{H}(16 \mathrm{~B})$ & 109.5 \\
\hline $\mathrm{C}(10)-\mathrm{C}(9)-\mathrm{H}(9)$ & 118.7 & $\mathrm{H}(16 \mathrm{~A})-\mathrm{C}(16)-\mathrm{H}(16 \mathrm{~B})$ & 109.5 \\
\hline$N(2)-C(10)-C(11)$ & $110.1(3)$ & $\mathrm{Si}(1)-\mathrm{C}(16)-\mathrm{H}(16 \mathrm{C})$ & 109.5 \\
\hline $\mathrm{N}(2)-\mathrm{C}(10)-\mathrm{C}(9)$ & $118.7(3)$ & $\mathrm{H}(16 \mathrm{~A})-\mathrm{C}(16)-\mathrm{H}(16 \mathrm{C})$ & 109.5 \\
\hline$C(11)-C(10)-C(9)$ & $131.2(3)$ & $\mathrm{H}(16 \mathrm{~B})-\mathrm{C}(16)-\mathrm{H}(16 \mathrm{C})$ & 109.5 \\
\hline$C(12)-C(11)-C(10)$ & $106.2(3)$ & $\mathrm{Si}(1)-\mathrm{C}(17)-\mathrm{H}(17 \mathrm{~A})$ & 109.5 \\
\hline $\mathrm{C}(12)-\mathrm{C}(11)-\mathrm{H}(11)$ & 126.9 & $\mathrm{Si}(1)-\mathrm{C}(17)-\mathrm{H}(17 \mathrm{~B})$ & 109.5 \\
\hline $\mathrm{C}(10)-\mathrm{C}\left(\begin{array}{ll}1 & 1\end{array}\right)-\mathrm{H}\left(\begin{array}{ll}1 & 1\end{array}\right)$ & 126.9 & $\mathrm{H}(17 \mathrm{~A})-\mathrm{C}(17)-\mathrm{H}(17 \mathrm{~B})$ & 109.5 \\
\hline$C(13)-C(12)-C(11)$ & $106.9(3)$ & $\mathrm{Si}(1)-\mathrm{C}(17)-\mathrm{H}(17 \mathrm{C})$ & 109.5 \\
\hline $\mathrm{C}(13)-\mathrm{C}(12)-\mathrm{H}(12)$ & 126.5 & $\mathrm{H}(17 \mathrm{~A})-\mathrm{C}(17)-\mathrm{H}(17 \mathrm{C})$ & 109.5 \\
\hline $\mathrm{C}(11)-\mathrm{C}(12)-\mathrm{H}(12)$ & 126.5 & $\mathrm{H}(17 \mathrm{~B})-\mathrm{C}(17)-\mathrm{H}(17 \mathrm{C})$ & 109.5 \\
\hline $\mathrm{N}(2)-\mathrm{C}(13)-\mathrm{C}(12)$ & $111.5(3)$ & $\mathrm{Si}(2)-\mathrm{C}(18)-\mathrm{Lu}$ & $126.32(15)$ \\
\hline $\mathrm{N}(2)-\mathrm{C}(13)-\mathrm{H}(13)$ & 124.3 & $\mathrm{Si}(2)-\mathrm{C}(18)-\mathrm{H}(18 \mathrm{~A})$ & 105.7 \\
\hline
\end{tabular}




\begin{tabular}{|c|c|c|c|}
\hline $\mathrm{Lu}-\mathrm{C}(18)-\mathrm{H}(18 \mathrm{~A})$ & 105.7 & $\mathrm{H}(22 \mathrm{~A})-\mathrm{C}(22)-\mathrm{H}(22 \mathrm{~B})$ & 108.5 \\
\hline $\mathrm{Si}(2)-\mathrm{C}(18)-\mathrm{H}(18 \mathrm{~B})$ & 105.7 & $C(24)-C(23)-C(22)$ & $108.1(3)$ \\
\hline $\mathrm{Lu}-\mathrm{C}(18)-\mathrm{H}(18 \mathrm{~B})$ & 105.7 & $\mathrm{C}(24)-\mathrm{C}(23)-\mathrm{H}(23 \mathrm{~A})$ & 110.1 \\
\hline $\mathrm{H}(18 \mathrm{~A})-\mathrm{C}(18)-\mathrm{H}(18 \mathrm{~B})$ & 106.2 & $\mathrm{C}(22)-\mathrm{C}(23)-\mathrm{H}(23 \mathrm{~A})$ & 110.1 \\
\hline $\mathrm{Si}(2)-\mathrm{C}(19)-\mathrm{H}(19 \mathrm{~A})$ & 109.5 & $\mathrm{C}(24)-\mathrm{C}(23)-\mathrm{H}(23 \mathrm{~B})$ & 110.1 \\
\hline $\mathrm{Si}(2)-\mathrm{C}(19)-\mathrm{H}(19 \mathrm{~B})$ & 109.5 & $\mathrm{C}(22)-\mathrm{C}(23)-\mathrm{H}(23 \mathrm{~B})$ & 110.1 \\
\hline $\mathrm{H}(19 \mathrm{~A})-\mathrm{C}(19)-\mathrm{H}(19 \mathrm{~B})$ & 109.5 & $\mathrm{H}(23 \mathrm{~A})-\mathrm{C}(23)-\mathrm{H}(23 \mathrm{~B})$ & 108.4 \\
\hline $\mathrm{Si}(2)-\mathrm{C}(19)-\mathrm{H}(19 \mathrm{C})$ & 109.5 & $\mathrm{C}(23)-\mathrm{C}(24)-\mathrm{C}(25)$ & $107.4(3)$ \\
\hline $\mathrm{H}(19 \mathrm{~A})-\mathrm{C}(19)-\mathrm{H}(19 \mathrm{C})$ & 109.5 & $\mathrm{C}(23)-\mathrm{C}(24)-\mathrm{H}(24 \mathrm{~A})$ & 110.2 \\
\hline $\mathrm{H}(19 \mathrm{~B})-\mathrm{C}(19)-\mathrm{H}(19 \mathrm{C})$ & 109.5 & $\mathrm{C}(25)-\mathrm{C}(24)-\mathrm{H}(24 \mathrm{~A})$ & 110.2 \\
\hline $\mathrm{Si}(2)-\mathrm{C}(20)-\mathrm{H}(20 \mathrm{~A})$ & 109.5 & $\mathrm{C}(23)-\mathrm{C}(24)-\mathrm{H}(24 \mathrm{~B})$ & 110.2 \\
\hline $\mathrm{Si}(2)-\mathrm{C}(20)-\mathrm{H}(20 \mathrm{~B})$ & 109.5 & $\mathrm{C}(25)-\mathrm{C}(24)-\mathrm{H}(24 \mathrm{~B})$ & 110.2 \\
\hline $\mathrm{H}(20 \mathrm{~A})-\mathrm{C}(20)-\mathrm{H}(20 \mathrm{~B})$ & 109.5 & $\mathrm{H}(24 \mathrm{~A})-\mathrm{C}(24)-\mathrm{H}(24 \mathrm{~B})$ & 108.5 \\
\hline $\mathrm{Si}(2)-\mathrm{C}(20)-\mathrm{H}(20 \mathrm{C})$ & 109.5 & $\mathrm{O}(2)-\mathrm{C}(25)-\mathrm{C}(24)$ & $106.3(3)$ \\
\hline $\mathrm{H}(20 \mathrm{~A})-\mathrm{C}(20)-\mathrm{H}(20 \mathrm{C})$ & 109.5 & $\mathrm{O}(2)-\mathrm{C}(25)-\mathrm{H}(25 \mathrm{~A})$ & 110.5 \\
\hline $\mathrm{H}(20 \mathrm{~B})-\mathrm{C}(20)-\mathrm{H}(20 \mathrm{C})$ & 109.5 & $\mathrm{C}(24)-\mathrm{C}(25)-\mathrm{H}(25 \mathrm{~A})$ & 110.5 \\
\hline $\mathrm{Si}(2)-\mathrm{C}(21)-\mathrm{H}(21 \mathrm{~A})$ & 109.5 & $\mathrm{O}(2)-\mathrm{C}(25)-\mathrm{H}(25 \mathrm{~B})$ & 110.5 \\
\hline $\mathrm{Si}(2)-\mathrm{C}(21)-\mathrm{H}(21 \mathrm{~B})$ & 109.5 & $\mathrm{C}(24)-\mathrm{C}(25)-\mathrm{H}(25 \mathrm{~B})$ & 110.5 \\
\hline $\mathrm{H}(21 \mathrm{~A})-\mathrm{C}(21)-\mathrm{H}(21 \mathrm{~B})$ & 109.5 & $\mathrm{H}(25 \mathrm{~A})-\mathrm{C}(25)-\mathrm{H}(25 \mathrm{~B})$ & 108.7 \\
\hline $\mathrm{Si}(2)-\mathrm{C}(21)-\mathrm{H}(21 \mathrm{C})$ & 109.5 & $\mathrm{O}(1)-\mathrm{C}(26)-\mathrm{C}(27 \mathrm{~B})$ & $107.1(4)$ \\
\hline $\mathrm{H}(21 \mathrm{~A})-\mathrm{C}(21)-\mathrm{H}(21 \mathrm{C})$ & 109.5 & $\mathrm{O}(1)-\mathrm{C}(26)-\mathrm{C}(27 \mathrm{~A})$ & $103.3(7)$ \\
\hline $\mathrm{H}(21 \mathrm{~B})-\mathrm{C}(21)-\mathrm{H}(21 \mathrm{C})$ & 109.5 & $C(27 B)-C(26)-C(27 A)$ & $33.1(5)$ \\
\hline $\mathrm{O}(2)-\mathrm{C}(22)-\mathrm{C}(23)$ & $107.2(3)$ & $\mathrm{O}(1)-\mathrm{C}(26)-\mathrm{H}(26 \mathrm{~A})$ & 111.1 \\
\hline $\mathrm{O}(2)-\mathrm{C}(22)-\mathrm{H}(22 \mathrm{~A})$ & 110.3 & $\mathrm{C}(27 \mathrm{~B})-\mathrm{C}(26)-\mathrm{H}(26 \mathrm{~A})$ & 79.5 \\
\hline $\mathrm{C}(23)-\mathrm{C}(22)-\mathrm{H}(22 \mathrm{~A})$ & 110.3 & $\mathrm{C}(27 \mathrm{~A})-\mathrm{C}(26)-\mathrm{H}(26 \mathrm{~A})$ & 111.1 \\
\hline $\mathrm{O}(2)-\mathrm{C}(22)-\mathrm{H}(22 \mathrm{~B})$ & 110.3 & $\mathrm{O}(1)-\mathrm{C}(26)-\mathrm{H}(26 \mathrm{~B})$ & 111.1 \\
\hline $\mathrm{C}(23)-\mathrm{C}(22)-\mathrm{H}(22 \mathrm{~B})$ & 110.3 & $\mathrm{C}(27 \mathrm{~B})-\mathrm{C}(26)-\mathrm{H}(26 \mathrm{~B})$ & 133.8 \\
\hline
\end{tabular}




\begin{tabular}{|c|c|c|c|}
\hline $\mathrm{C}(27 \mathrm{~A})-\mathrm{C}(26)-\mathrm{H}(26 \mathrm{~B})$ & 111.1 & $\mathrm{H}(29 \mathrm{~B})-\mathrm{C}(29)-\mathrm{H}(29 \mathrm{C})$ & 18.0 \\
\hline $\mathrm{H}(26 \mathrm{~A})-\mathrm{C}(26)-\mathrm{H}(26 \mathrm{~B})$ & 109.1 & $\mathrm{C}(28 \mathrm{~A})-\mathrm{C}(29)-\mathrm{H}(29 \mathrm{D})$ & 90.1 \\
\hline $\mathrm{O}(1)-\mathrm{C}(26)-\mathrm{H}(26 \mathrm{C})$ & 110.2 & $\mathrm{O}(1)-\mathrm{C}(29)-\mathrm{H}(29 \mathrm{D})$ & 110.8 \\
\hline $\mathrm{C}(27 \mathrm{~B})-\mathrm{C}(26)-\mathrm{H}(26 \mathrm{C})$ & 112.1 & $\mathrm{C}(28 \mathrm{~B})-\mathrm{C}(29)-\mathrm{H}(29 \mathrm{D})$ & 108.6 \\
\hline $\mathrm{C}(27 \mathrm{~A})-\mathrm{C}(26)-\mathrm{H}(26 \mathrm{C})$ & 139.2 & $\mathrm{H}(29 \mathrm{~A})-\mathrm{C}(29)-\mathrm{H}(29 \mathrm{D})$ & 20.9 \\
\hline $\mathrm{H}(26 \mathrm{~A})-\mathrm{C}(26)-\mathrm{H}(26 \mathrm{C})$ & 34.7 & H(29B)-C(29)-H(29D) & 124.0 \\
\hline $\mathrm{H}(26 \mathrm{~B})-\mathrm{C}(26)-\mathrm{H}(26 \mathrm{C})$ & 77.9 & $\mathrm{H}(29 \mathrm{C})-\mathrm{C}(29)-\mathrm{H}(29 \mathrm{D})$ & 108.8 \\
\hline $\mathrm{O}(1)-\mathrm{C}(26)-\mathrm{H}(26 \mathrm{D})$ & 110.2 & $\mathrm{C}(28 \mathrm{~A})-\mathrm{C}(27 \mathrm{~A})-\mathrm{C}(26)$ & $103.0(17)$ \\
\hline $\mathrm{C}(27 \mathrm{~B})-\mathrm{C}(26)-\mathrm{H}(26 \mathrm{D})$ & 108.8 & $\mathrm{C}(28 \mathrm{~A})-\mathrm{C}(27 \mathrm{~A})-\mathrm{H}(27 \mathrm{~A})$ & 111.2 \\
\hline $\mathrm{C}(27 \mathrm{~A})-\mathrm{C}(26)-\mathrm{H}(26 \mathrm{D})$ & 80.0 & $\mathrm{C}(26)-\mathrm{C}(27 \mathrm{~A})-\mathrm{H}(27 \mathrm{~A})$ & 111.2 \\
\hline $\mathrm{H}(26 \mathrm{~A})-\mathrm{C}(26)-\mathrm{H}(26 \mathrm{D})$ & 132.9 & $\mathrm{C}(28 \mathrm{~A})-\mathrm{C}(27 \mathrm{~A})-\mathrm{H}(27 \mathrm{~B})$ & 111.2 \\
\hline $\mathrm{H}(26 \mathrm{~B})-\mathrm{C}(26)-\mathrm{H}(26 \mathrm{D})$ & 32.9 & $\mathrm{C}(26)-\mathrm{C}(27 \mathrm{~A})-\mathrm{H}(27 \mathrm{~B})$ & 111.2 \\
\hline $\mathrm{H}(26 \mathrm{C})-\mathrm{C}(26)-\mathrm{H}(26 \mathrm{D})$ & 108.5 & $\mathrm{H}(27 \mathrm{~A})-\mathrm{C}(27 \mathrm{~A})-\mathrm{H}(27 \mathrm{~B})$ & 109.1 \\
\hline $\mathrm{C}(28 \mathrm{~A})-\mathrm{C}(29)-\mathrm{O}(1)$ & $111.9(8)$ & $C(26)-C(27 B)-C(28 B)$ & $98.1(9)$ \\
\hline $\mathrm{C}(28 \mathrm{~A})-\mathrm{C}(29)-\mathrm{C}(28 \mathrm{~B})$ & $18.6(14)$ & $\mathrm{C}(26)-\mathrm{C}(27 \mathrm{~B})-\mathrm{H}(27 \mathrm{C})$ & 112.1 \\
\hline $\mathrm{O}(1)-\mathrm{C}(29)-\mathrm{C}(28 \mathrm{~B})$ & $102.6(5)$ & $\mathrm{C}(28 \mathrm{~B})-\mathrm{C}(27 \mathrm{~B})-\mathrm{H}(27 \mathrm{C})$ & 112.1 \\
\hline $\mathrm{C}(28 \mathrm{~A})-\mathrm{C}(29)-\mathrm{H}(29 \mathrm{~A})$ & 109.2 & $\mathrm{C}(26)-\mathrm{C}(27 \mathrm{~B})-\mathrm{H}(27 \mathrm{D})$ & 112.1 \\
\hline $\mathrm{O}(1)-\mathrm{C}(29)-\mathrm{H}(29 \mathrm{~A})$ & 109.2 & $\mathrm{C}(28 \mathrm{~B})-\mathrm{C}(27 \mathrm{~B})-\mathrm{H}(27 \mathrm{D})$ & 112.1 \\
\hline $\mathrm{C}(28 \mathrm{~B})-\mathrm{C}(29)-\mathrm{H}(29 \mathrm{~A})$ & 127.7 & $\mathrm{H}(27 \mathrm{C})-\mathrm{C}(27 \mathrm{~B})-\mathrm{H}(27 \mathrm{D})$ & 109.8 \\
\hline $\mathrm{C}(28 \mathrm{~A})-\mathrm{C}(29)-\mathrm{H}(29 \mathrm{~B})$ & 109.2 & $\mathrm{C}(29)-\mathrm{C}(28 \mathrm{~A})-\mathrm{C}(27 \mathrm{~A})$ & $103.1(19)$ \\
\hline $\mathrm{O}(1)-\mathrm{C}(29)-\mathrm{H}(29 \mathrm{~B})$ & 109.2 & $\mathrm{C}(29)-\mathrm{C}(28 \mathrm{~A})-\mathrm{H}(28 \mathrm{~A})$ & 111.1 \\
\hline $\mathrm{C}(28 \mathrm{~B})-\mathrm{C}(29)-\mathrm{H}(29 \mathrm{~B})$ & 98.9 & $\mathrm{C}(27 \mathrm{~A})-\mathrm{C}(28 \mathrm{~A})-\mathrm{H}(28 \mathrm{~A})$ & 111.1 \\
\hline $\mathrm{H}(29 \mathrm{~A})-\mathrm{C}(29)-\mathrm{H}(29 \mathrm{~B})$ & 107.9 & $\mathrm{C}(29)-\mathrm{C}(28 \mathrm{~A})-\mathrm{H}(28 \mathrm{~B})$ & 111.1 \\
\hline $\mathrm{C}(28 \mathrm{~A})-\mathrm{C}(29)-\mathrm{H}(29 \mathrm{C})$ & 122.3 & $\mathrm{C}(27 \mathrm{~A})-\mathrm{C}(28 \mathrm{~A})-\mathrm{H}(28 \mathrm{~B})$ & 111.1 \\
\hline $\mathrm{O}(1)-\mathrm{C}(29)-\mathrm{H}(29 \mathrm{C})$ & 110.8 & $\mathrm{H}(28 \mathrm{~A})-\mathrm{C}(28 \mathrm{~A})-\mathrm{H}(28 \mathrm{~B})$ & 109.1 \\
\hline $\mathrm{C}(28 \mathrm{~B})-\mathrm{C}(29)-\mathrm{H}(29 \mathrm{C})$ & 115.1 & $\mathrm{C}(29)-\mathrm{C}(28 \mathrm{~B})-\mathrm{C}(27 \mathrm{~B})$ & $99.7(11)$ \\
\hline $\mathrm{H}(29 \mathrm{~A})-\mathrm{C}(29)-\mathrm{H}(29 \mathrm{C})$ & 91.0 & $\mathrm{C}(29)-\mathrm{C}(28 \mathrm{~B})-\mathrm{H}(28 \mathrm{C})$ & 111.8 \\
\hline
\end{tabular}




\begin{tabular}{llll}
$\mathrm{C}(27 \mathrm{~B})-\mathrm{C}(28 \mathrm{~B})-\mathrm{H}(28 \mathrm{C})$ & 111.8 & $\mathrm{C}(27 \mathrm{~B})-\mathrm{C}(28 \mathrm{~B})-\mathrm{H}(28 \mathrm{D})$ & 111.8 \\
$\mathrm{C}(29)-\mathrm{C}(28 \mathrm{~B})-\mathrm{H}(28 \mathrm{D})$ & 111.8 & $\mathrm{H}(28 \mathrm{C})-\mathrm{C}(28 \mathrm{~B})-\mathrm{H}(28 \mathrm{D})$ & 109.6 \\
& & & \\
\hline
\end{tabular}

\#---End of complex 1a 
Stable 6. Crystal data and structure refinement for $\mathbf{1 b}$

Identification code

Empirical formula

Formula weight

Temperature

Wavelength

Crystal system

Space group

Unit cell dimensions

Volume

Z

Density (calculated)

Absorption coefficient

$\mathrm{F}(000)$

Crystal size

Theta range for data collection

Index ranges

Reflections collected

Independent reflections

Completeness to theta $=26.04^{\circ}$

Absorption correction

Max. and min. transmission

Refinement method

Data / restraints / parameters r025

C25 H43 Sc N2 O Si2

488.75

187(2) K

$0.71073 \AA$

Monoclinic

$\mathrm{P} 2_{1} / \mathrm{n}$

$\mathrm{a}=11.5805(8) \AA$

$\alpha=90^{\circ}$.

$\mathrm{b}=14.0152(10) \AA$

$\beta=90.5080(10)^{\circ}$.

$c=17.7458(13) \AA$

$\gamma=90^{\circ}$.

2880.1(4) $\AA^{3}$

4

$1.127 \mathrm{Mg} / \mathrm{m}^{3}$

$0.356 \mathrm{~mm}^{-1}$

1056

$0.25 \times 0.18 \times 0.16 \mathrm{~mm}^{3}$

1.85 to $26.04^{\circ}$.

$-14<=\mathrm{h}<=13,-17<=\mathrm{k}<=17,-21<=\mathrm{l}<=13$

15906

$5680[\mathrm{R}($ int $)=0.0305]$

$99.8 \%$

Semi-empirical from equivalents

0.9452 and 0.9162

Full-matrix least-squares on $\mathrm{F}^{2}$

$5680 / 0 / 288$ 
Goodness-of-fit on $\mathrm{F}^{2}$

Final $\mathrm{R}$ indices [I $>2 \operatorname{sigma}(\mathrm{I})]$

$\mathrm{R}$ indices (all data)

Largest diff. peak and hole
1.021

$\mathrm{R} 1=0.0416, \mathrm{wR} 2=0.1032$

$\mathrm{R} 1=0.0563, \mathrm{wR} 2=0.1114$

0.350 and $-0.227 \mathrm{e}^{-3}$ 
Stable 7. Atomic coordinates $\left(\begin{array}{ll}x & 1^{4}\end{array}\right)$ and equivalent isotropic displacement parameters $\left(\AA^{2} \times 10^{3}\right)$ for $1 \mathrm{~b}$. $U(\mathrm{eq})$ is defined as one third of the trace of the orthogonalized Uij tensor.

\begin{tabular}{|c|c|c|c|c|}
\hline & $\mathrm{x}$ & $\mathrm{y}$ & z & $\mathrm{U}(\mathrm{eq})$ \\
\hline $\mathrm{Sc}$ & $3114(1)$ & $7719(1)$ & $1573(1)$ & $26(1)$ \\
\hline $\operatorname{Si}(1)$ & $43(1)$ & 7591(1) & $1769(1)$ & $33(1)$ \\
\hline $\operatorname{Si}(2)$ & $4804(1)$ & $6939(1)$ & $-42(1)$ & $38(1)$ \\
\hline $\mathrm{N}(1)$ & $3951(1)$ & $9105(1)$ & $2002(1)$ & $28(1)$ \\
\hline $\mathrm{N}(2)$ & $2545(1)$ & $8831(1)$ & $789(1)$ & $31(1)$ \\
\hline $\mathrm{O}$ & $4185(1)$ & $7044(1)$ & $2453(1)$ & $36(1)$ \\
\hline $\mathrm{C}(1)$ & $1898(2)$ & $8934(2)$ & $160(1)$ & $39(1)$ \\
\hline$C(2)$ & $1876(2)$ & $9875(2)$ & $-86(1)$ & $41(1)$ \\
\hline$C(3)$ & $2567(2)$ & $10381(2)$ & $409(1)$ & $39(1)$ \\
\hline$C(4)$ & $2969(2)$ & $9730(1)$ & $946(1)$ & $29(1)$ \\
\hline$C(5)$ & $3699(2)$ & $9837(1)$ & $1582(1)$ & $30(1)$ \\
\hline$C(6)$ & $4723(2)$ & $9252(1)$ & $2630(1)$ & $31(1)$ \\
\hline$C(7)$ & $4277(2)$ & $9273(2)$ & $3357(1)$ & $40(1)$ \\
\hline $\mathrm{C}(8)$ & $5056(3)$ & $9359(2)$ & $3957(1)$ & $57(1)$ \\
\hline$C(9)$ & $6230(3)$ & $9429(2)$ & $3837(2)$ & $63(1)$ \\
\hline$C(10)$ & $6648(2)$ & $9425(2)$ & $3119(2)$ & $56(1)$ \\
\hline$C(11)$ & $5913(2)$ & $9342(2)$ & $2497(1)$ & $41(1)$ \\
\hline$C(12)$ & $6395(2)$ & $9356(2)$ & $1712(1)$ & $54(1)$ \\
\hline$C(13)$ & $3002(2)$ & $9229(2)$ & $3488(1)$ & $56(1)$ \\
\hline$C(14)$ & $1483(2)$ & $7197(1)$ & 2083(1) & $34(1)$ \\
\hline
\end{tabular}




$\begin{array}{lrrrr}\mathrm{C}(15) & -28(2) & 8927(2) & 1830(1) & 49(1) \\ \mathrm{C}(16) & -1177(2) & 7123(2) & 2353(2) & 52(1) \\ \mathrm{C}(17) & -263(2) & 7184(2) & 783(1) & 63(1) \\ \mathrm{C}(18) & 3701(2) & 6733(1) & 681(1) & 35(1) \\ \mathrm{C}(19) & 4648(2) & 8152(2) & -472(2) & 56(1) \\ \mathrm{C}(20) & 6306(2) & 6855(2) & 359(2) & 56(1) \\ \mathrm{C}(21) & 4734(2) & 6058(2) & -841(1) & 54(1) \\ \mathrm{C}(22) & 3819(2) & 6566(2) & 3134(1) & 48(1) \\ \mathrm{C}(23) & 4841(2) & 6598(2) & 3658(1) & 47(1) \\ \mathrm{C}(24) & 5867(2) & 6670(2) & 3150(2) & 63(1) \\ \mathrm{C}(25) & 5395(2) & 6798(2) & 2368(1) & 51(1)\end{array}$


Stable 8. Anisotropic displacement parameters $\left(\AA^{2} \times 10^{3}\right)$ for $1 b$. The anisotropic displacement factor exponent takes the form: $-2 \pi^{2}\left[h^{2} a^{* 2} U^{11}+\ldots+2 h\right.$ k a* $\left.\mathbf{b}^{*} \mathbf{U}^{12}\right]$

\begin{tabular}{|c|c|c|c|c|c|c|}
\hline & U11 & $\mathrm{U} 22$ & U33 & U23 & U13 & U12 \\
\hline $\mathrm{Sc}$ & $24(1)$ & $30(1)$ & $26(1)$ & $4(1)$ & $-2(1)$ & $0(1)$ \\
\hline $\operatorname{Si}(1)$ & $25(1)$ & $44(1)$ & $32(1)$ & $5(1)$ & $-3(1)$ & $-3(1)$ \\
\hline $\operatorname{Si}(2)$ & $34(1)$ & $45(1)$ & $34(1)$ & $-4(1)$ & $2(1)$ & $-2(1)$ \\
\hline $\mathrm{N}(1)$ & $26(1)$ & $33(1)$ & $24(1)$ & $2(1)$ & $-3(1)$ & $0(1)$ \\
\hline $\mathrm{N}(2)$ & $29(1)$ & $36(1)$ & $27(1)$ & $7(1)$ & $-5(1)$ & $-2(1)$ \\
\hline $\mathrm{O}$ & $29(1)$ & $43(1)$ & $35(1)$ & 13(1) & $-3(1)$ & $4(1)$ \\
\hline $\mathrm{C}(1)$ & $37(1)$ & $48(1)$ & $30(1)$ & $6(1)$ & $-10(1)$ & $-7(1)$ \\
\hline$C(2)$ & $36(1)$ & $56(1)$ & $29(1)$ & $15(1)$ & $-7(1)$ & $2(1)$ \\
\hline$C(3)$ & $45(1)$ & $35(1)$ & $39(1)$ & $10(1)$ & $-1(1)$ & $4(1)$ \\
\hline$C(4)$ & $28(1)$ & $34(1)$ & $26(1)$ & $4(1)$ & $2(1)$ & $4(1)$ \\
\hline$C(5)$ & $30(1)$ & $32(1)$ & $29(1)$ & $-1(1)$ & 1(1) & $0(1)$ \\
\hline$C(6)$ & $36(1)$ & $29(1)$ & $28(1)$ & $2(1)$ & $-8(1)$ & $-2(1)$ \\
\hline$C(7)$ & $55(1)$ & $38(1)$ & $28(1)$ & $0(1)$ & $-1(1)$ & $-6(1)$ \\
\hline $\mathrm{C}(8)$ & $91(2)$ & $52(1)$ & $27(1)$ & 2(1) & $-12(1)$ & $-8(1)$ \\
\hline $\mathrm{C}(9)$ & $75(2)$ & $63(2)$ & $51(2)$ & 2(1) & $-38(2)$ & $-6(1)$ \\
\hline$C(10)$ & $45(1)$ & $63(2)$ & $61(2)$ & $4(1)$ & $-24(1)$ & $-4(1)$ \\
\hline$C(11)$ & $36(1)$ & $44(1)$ & $42(1)$ & $3(1)$ & $-10(1)$ & $-1(1)$ \\
\hline$C(12)$ & $32(1)$ & $73(2)$ & $58(2)$ & $4(1)$ & $4(1)$ & $0(1)$ \\
\hline$C(13)$ & $65(2)$ & $65(2)$ & $39(1)$ & $-4(1)$ & $17(1)$ & $-10(1)$ \\
\hline$C(14)$ & $31(1)$ & $32(1)$ & $40(1)$ & $5(1)$ & $0(1)$ & $0(1)$ \\
\hline
\end{tabular}




$\begin{array}{lcccccc}\mathrm{C}(15) & 46(1) & 49(1) & 53(2) & 13(1) & 6(1) & 12(1) \\ \mathrm{C}(16) & 33(1) & 71(2) & 52(2) & 14(1) & 5(1) & -8(1) \\ \mathrm{C}(17) & 60(2) & 89(2) & 38(1) & 1(1) & -8(1) & -31(2) \\ \mathrm{C}(18) & 33(1) & 35(1) & 37(1) & -2(1) & -1(1) & -1(1) \\ \mathrm{C}(19) & 57(2) & 59(2) & 51(2) & 8(1) & 15(1) & -6(1) \\ \mathrm{C}(20) & 39(1) & 79(2) & 51(2) & -12(1) & 2(1) & -2(1) \\ \mathrm{C}(21) & 53(2) & 65(2) & 43(1) & -13(1) & 1(1) & 0(1) \\ \mathrm{C}(22) & 38(1) & 68(2) & 38(1) & 20(1) & -3(1) & 4(1) \\ \mathrm{C}(23) & 50(1) & 51(1) & 39(1) & 10(1) & -13(1) & 8(1) \\ \mathrm{C}(24) & 37(1) & 89(2) & 62(2) & 32(2) & -13(1) & -4(1) \\ \mathrm{C}(25) & 34(1) & 70(2) & 50(2) & 15(1) & -2(1) & 11(1)\end{array}$


Stable 9. Bond lengths $[\AA]]$ and angles $\left[^{\circ}\right]$ for $1 b$

\begin{tabular}{|c|c|c|c|}
\hline $\mathrm{Sc}-\mathrm{N}(2)$ & $2.1872(16)$ & $C(4)-C(5)$ & $1.411(3)$ \\
\hline $\mathrm{Sc}-\mathrm{O}$ & $2.1990(14)$ & $\mathrm{C}(5)-\mathrm{H}(5)$ & 0.9300 \\
\hline Sc-C(18) & $2.212(2)$ & $C(6)-C(7)$ & $1.395(3)$ \\
\hline Sc-C(14) & $2.226(2)$ & $C(6)-C(11)$ & $1.405(3)$ \\
\hline $\mathrm{Sc}-\mathrm{N}(1)$ & $2.2977(16)$ & $C(7)-C(8)$ & $1.394(3)$ \\
\hline $\mathrm{Si}(1)-\mathrm{C}(14)$ & $1.838(2)$ & $C(7)-C(13)$ & $1.498(3)$ \\
\hline $\mathrm{Si}(1)-\mathrm{C}(17)$ & $1.870(3)$ & $\mathrm{C}(8)-\mathrm{C}(9)$ & $1.382(4)$ \\
\hline $\mathrm{Si}(1)-\mathrm{C}(15)$ & $1.877(2)$ & $\mathrm{C}(8)-\mathrm{H}(8)$ & 0.9300 \\
\hline $\operatorname{Si}(1)-C(16)$ & $1.878(2)$ & $\mathrm{C}(9)-\mathrm{C}(10)$ & $1.367(4)$ \\
\hline $\mathrm{Si}(2)-\mathrm{C}(18)$ & $1.842(2)$ & $\mathrm{C}(9)-\mathrm{H}(9)$ & 0.9300 \\
\hline $\mathrm{Si}(2)-\mathrm{C}(19)$ & $1.871(3)$ & $\mathrm{C}(10)-\mathrm{C}(11)$ & $1.392(3)$ \\
\hline $\mathrm{Si}(2)-\mathrm{C}(20)$ & $1.877(2)$ & $\mathrm{C}(10)-\mathrm{H}(10)$ & 0.9300 \\
\hline $\mathrm{Si}(2)-\mathrm{C}(21)$ & $1.881(2)$ & $\mathrm{C}(11)-\mathrm{C}(12)$ & $1.507(3)$ \\
\hline $\mathrm{N}(1)-\mathrm{C}(5)$ & $1.300(2)$ & $\mathrm{C}(12)-\mathrm{H}(12 \mathrm{~A})$ & 0.9600 \\
\hline $\mathrm{N}(1)-\mathrm{C}(6)$ & $1.437(2)$ & $\mathrm{C}(12)-\mathrm{H}(12 \mathrm{~B})$ & 0.9600 \\
\hline $\mathrm{N}(2)-\mathrm{C}(1)$ & $1.347(2)$ & $\mathrm{C}(12)-\mathrm{H}(12 \mathrm{C})$ & 0.9600 \\
\hline $\mathrm{N}(2)-\mathrm{C}(4)$ & $1.380(2)$ & $\mathrm{C}(13)-\mathrm{H}(13 \mathrm{~A})$ & 0.9600 \\
\hline $\mathrm{O}-\mathrm{C}(22)$ & $1.449(2)$ & $\mathrm{C}(13)-\mathrm{H}(13 \mathrm{~B})$ & 0.9600 \\
\hline $\mathrm{O}-\mathrm{C}(25)$ & $1.453(3)$ & $\mathrm{C}(13)-\mathrm{H}(13 \mathrm{C})$ & 0.9600 \\
\hline$C(1)-C(2)$ & $1.389(3)$ & $\mathrm{C}(14)-\mathrm{H}(14 \mathrm{~A})$ & 0.9700 \\
\hline $\mathrm{C}(1)-\mathrm{H}(1)$ & 0.9300 & $\mathrm{C}(14)-\mathrm{H}(14 \mathrm{~B})$ & 0.9700 \\
\hline$C(2)-C(3)$ & $1.380(3)$ & $\mathrm{C}(15)-\mathrm{H}(15 \mathrm{~A})$ & 0.9600 \\
\hline $\mathrm{C}(2)-\mathrm{H}(2)$ & 0.9300 & $\mathrm{C}(15)-\mathrm{H}(15 \mathrm{~B})$ & 0.9600 \\
\hline$C(3)-C(4)$ & $1.396(3)$ & $\mathrm{C}(15)-\mathrm{H}(15 \mathrm{C})$ & 0.9600 \\
\hline $\mathrm{C}(3)-\mathrm{H}(3)$ & 0.9300 & $\mathrm{C}(16)-\mathrm{H}(16 \mathrm{~A})$ & 0.9600 \\
\hline
\end{tabular}




\begin{tabular}{|c|c|c|c|}
\hline$C(16)-H(16 B)$ & 0.9600 & $\mathrm{C}(21)-\mathrm{H}(21 \mathrm{~B})$ & 0.9600 \\
\hline$C(16)-H(16 C)$ & 0.9600 & $\mathrm{C}(21)-\mathrm{H}(21 \mathrm{C})$ & 0.9600 \\
\hline $\mathrm{C}(17)-\mathrm{H}(17 \mathrm{~A})$ & 0.9600 & $\mathrm{C}(22)-\mathrm{C}(23)$ & $1.499(3)$ \\
\hline $\mathrm{C}(17)-\mathrm{H}(17 \mathrm{~B})$ & 0.9600 & $\mathrm{C}(22)-\mathrm{H}(22 \mathrm{~A})$ & 0.9700 \\
\hline $\mathrm{C}(17)-\mathrm{H}(17 \mathrm{C})$ & 0.9600 & $\mathrm{C}(22)-\mathrm{H}(22 \mathrm{~B})$ & 0.9700 \\
\hline $\mathrm{C}(18)-\mathrm{H}(18 \mathrm{~A})$ & 0.9700 & $\mathrm{C}(23)-\mathrm{C}(24)$ & $1.502(3)$ \\
\hline $\mathrm{C}(18)-\mathrm{H}(18 \mathrm{~B})$ & 0.9700 & $\mathrm{C}(23)-\mathrm{H}(23 \mathrm{~A})$ & 0.9700 \\
\hline $\mathrm{C}(19)-\mathrm{H}(19 \mathrm{~A})$ & 0.9600 & $\mathrm{C}(23)-\mathrm{H}(23 \mathrm{~B})$ & 0.9700 \\
\hline$C(19)-H(19 B)$ & 0.9600 & $C(24)-C(25)$ & $1.498(3)$ \\
\hline $\mathrm{C}(19)-\mathrm{H}(19 \mathrm{C})$ & 0.9600 & $\mathrm{C}(24)-\mathrm{H}(24 \mathrm{~A})$ & 0.9700 \\
\hline $\mathrm{C}(20)-\mathrm{H}(20 \mathrm{~A})$ & 0.9600 & $\mathrm{C}(24)-\mathrm{H}(24 \mathrm{~B})$ & 0.9700 \\
\hline $\mathrm{C}(20)-\mathrm{H}(20 \mathrm{~B})$ & 0.9600 & $\mathrm{C}(25)-\mathrm{H}(25 \mathrm{~A})$ & 0.9700 \\
\hline $\mathrm{C}(20)-\mathrm{H}(20 \mathrm{C})$ & 0.9600 & $\mathrm{C}(25)-\mathrm{H}(25 \mathrm{~B})$ & 0.9700 . \\
\hline $\mathrm{C}(21)-\mathrm{H}(21 \mathrm{~A})$ & 0.9600 & & \\
\hline $\mathrm{N}(2)-\mathrm{Sc}-\mathrm{O}$ & $157.41(6)$ & $\mathrm{C}(14)-\mathrm{Si}(1)-\mathrm{C}(16)$ & $114.34(11)$ \\
\hline $\mathrm{N}(2)-\mathrm{Sc}-\mathrm{C}(18)$ & $94.77(7)$ & $\mathrm{C}(17)-\mathrm{Si}(1)-\mathrm{C}(16)$ & $105.79(12)$ \\
\hline O-Sc-C(18) & $93.66(7)$ & $C(15)-\operatorname{Si}(1)-C(16)$ & $106.46(11)$ \\
\hline $\mathrm{N}(2)-\mathrm{Sc}-\mathrm{C}(14)$ & $103.91(7)$ & $\mathrm{C}(18)-\mathrm{Si}(2)-\mathrm{C}(19)$ & $111.22(10)$ \\
\hline $\mathrm{O}-\mathrm{Sc}-\mathrm{C}(14)$ & $92.59(7)$ & $\mathrm{C}(18)-\mathrm{Si}(2)-\mathrm{C}(20)$ & $111.85(11)$ \\
\hline $\mathrm{C}(18)-\mathrm{Sc}-\mathrm{C}(14)$ & $110.64(8)$ & $\mathrm{C}(19)-\mathrm{Si}(2)-\mathrm{C}(20)$ & $107.30(13)$ \\
\hline $\mathrm{N}(2)-\mathrm{Sc}-\mathrm{N}(1)$ & $74.46(6)$ & $\mathrm{C}(18)-\mathrm{Si}(2)-\mathrm{C}(21)$ & $113.35(11)$ \\
\hline $\mathrm{O}-\mathrm{Sc}-\mathrm{N}(1)$ & $84.00(5)$ & $C(19)-S i(2)-C(21)$ & $106.58(12)$ \\
\hline $\mathrm{C}(18)-\mathrm{Sc}-\mathrm{N}(1)$ & $129.36(7)$ & $C(20)-S i(2)-C(21)$ & $106.15(11)$ \\
\hline $\mathrm{C}(14)-\mathrm{Sc}-\mathrm{N}(1)$ & $120.00(7)$ & $\mathrm{C}(5)-\mathrm{N}(1)-\mathrm{C}(6)$ & $117.83(16)$ \\
\hline$C(14)-\operatorname{Si}(1)-C(17)$ & $110.82(12)$ & $\mathrm{C}(5)-\mathrm{N}(1)-\mathrm{Sc}$ & $112.64(12)$ \\
\hline$C(14)-S i(1)-C(15)$ & $108.80(10)$ & $\mathrm{C}(6)-\mathrm{N}(1)-\mathrm{Sc}$ & $129.47(12)$ \\
\hline$C(17)-S i(1)-C(15)$ & $110.52(12)$ & $\mathrm{C}(1)-\mathrm{N}(2)-\mathrm{C}(4)$ & $105.34(16)$ \\
\hline
\end{tabular}




\begin{tabular}{|c|c|c|c|}
\hline$C(1)-N(2)-S c$ & $139.99(14)$ & $\mathrm{C}(9)-\mathrm{C}(8)-\mathrm{H}(8)$ & 119.4 \\
\hline$C(4)-N(2)-S c$ & $114.66(12)$ & $\mathrm{C}(7)-\mathrm{C}(8)-\mathrm{H}(8)$ & 119.4 \\
\hline $\mathrm{C}(22)-\mathrm{O}-\mathrm{C}(25)$ & $105.46(16)$ & $C(10)-C(9)-C(8)$ & $120.0(2)$ \\
\hline $\mathrm{C}(22)-\mathrm{O}-\mathrm{Sc}$ & $128.64(12)$ & $\mathrm{C}(10)-\mathrm{C}(9)-\mathrm{H}(9)$ & 120.0 \\
\hline $\mathrm{C}(25)-\mathrm{O}-\mathrm{Sc}$ & $124.54(13)$ & $\mathrm{C}(8)-\mathrm{C}(9)-\mathrm{H}(9)$ & 120.0 \\
\hline$N(2)-C(1)-C(2)$ & $111.74(19)$ & $C(9)-C(10)-C(11)$ & $121.3(2)$ \\
\hline $\mathrm{N}(2)-\mathrm{C}(1)-\mathrm{H}(1)$ & 124.1 & $\mathrm{C}(9)-\mathrm{C}(10)-\mathrm{H}(10)$ & 119.4 \\
\hline $\mathrm{C}(2)-\mathrm{C}(1)-\mathrm{H}(1)$ & 124.1 & $\mathrm{C}\left(\begin{array}{ll}1 & 1\end{array}\right)-\mathrm{C}\left(\begin{array}{ll}1 & 0\end{array}\right)-\mathrm{H}\left(\begin{array}{ll}1 & 0\end{array}\right)$ & 119.4 \\
\hline$C(3)-C(2)-C(1)$ & $106.20(18)$ & $\mathrm{C}(10)-\mathrm{C}(11)-\mathrm{C}(6)$ & $118.0(2)$ \\
\hline $\mathrm{C}(3)-\mathrm{C}(2)-\mathrm{H}(2)$ & 126.9 & $C(10)-C(11)-C(12)$ & $120.1(2)$ \\
\hline $\mathrm{C}(1)-\mathrm{C}(2)-\mathrm{H}(2)$ & 126.9 & $C(6)-C(11)-C(12)$ & $121.9(2)$ \\
\hline$C(2)-C(3)-C(4)$ & $106.71(19)$ & $\mathrm{C}(11)-\mathrm{C}(12)-\mathrm{H}(12 \mathrm{~A})$ & 109.5 \\
\hline $\mathrm{C}(2)-\mathrm{C}(3)-\mathrm{H}(3)$ & 126.6 & $\mathrm{C}(11)-\mathrm{C}(12)-\mathrm{H}(12 \mathrm{~B})$ & 109.5 \\
\hline $\mathrm{C}(4)-\mathrm{C}(3)-\mathrm{H}(3)$ & 126.6 & $\mathrm{H}(12 \mathrm{~A})-\mathrm{C}(12)-\mathrm{H}(12 \mathrm{~B})$ & 109.5 \\
\hline$N(2)-C(4)-C(3)$ & $110.01(18)$ & $\mathrm{C}(11)-\mathrm{C}(12)-\mathrm{H}(12 \mathrm{C})$ & 109.5 \\
\hline$N(2)-C(4)-C(5)$ & $117.88(16)$ & $\mathrm{H}(12 \mathrm{~A})-\mathrm{C}(12)-\mathrm{H}(12 \mathrm{C})$ & 109.5 \\
\hline$C(3)-C(4)-C(5)$ & $132.11(19)$ & $\mathrm{H}(12 \mathrm{~B})-\mathrm{C}(12)-\mathrm{H}(12 \mathrm{C})$ & 109.5 \\
\hline$N(1)-C(5)-C(4)$ & $120.35(17)$ & $\mathrm{C}(7)-\mathrm{C}(13)-\mathrm{H}(13 \mathrm{~A})$ & 109.5 \\
\hline $\mathrm{N}(1)-\mathrm{C}(5)-\mathrm{H}(5)$ & 119.8 & $\mathrm{C}(7)-\mathrm{C}(13)-\mathrm{H}(13 \mathrm{~B})$ & 109.5 \\
\hline $\mathrm{C}(4)-\mathrm{C}(5)-\mathrm{H}(5)$ & 119.8 & $\mathrm{H}(13 \mathrm{~A})-\mathrm{C}(13)-\mathrm{H}(13 \mathrm{~B})$ & 109.5 \\
\hline$C(7)-C(6)-C(11)$ & $121.6(2)$ & $\mathrm{C}(7)-\mathrm{C}(13)-\mathrm{H}(13 \mathrm{C})$ & 109.5 \\
\hline$C(7)-C(6)-N(1)$ & $119.14(18)$ & $\mathrm{H}(13 \mathrm{~A})-\mathrm{C}(13)-\mathrm{H}(13 \mathrm{C})$ & 109.5 \\
\hline $\mathrm{C}(11)-\mathrm{C}(6)-\mathrm{N}(1)$ & $119.22(18)$ & $\mathrm{H}(13 \mathrm{~B})-\mathrm{C}(13)-\mathrm{H}(13 \mathrm{C})$ & 109.5 \\
\hline$C(8)-C(7)-C(6)$ & $117.8(2)$ & $\mathrm{Si}(1)-\mathrm{C}(14)-\mathrm{Sc}$ & $123.31(10)$ \\
\hline$C(8)-C(7)-C(13)$ & $121.1(2)$ & $\mathrm{Si}(1)-\mathrm{C}(14)-\mathrm{H}(14 \mathrm{~A})$ & 106.5 \\
\hline$C(6)-C(7)-C(13)$ & $121.1(2)$ & $\mathrm{Sc}-\mathrm{C}(14)-\mathrm{H}(14 \mathrm{~A})$ & 106.5 \\
\hline$C(9)-C(8)-C(7)$ & $121.3(2)$ & $\mathrm{Si}(1)-\mathrm{C}(14)-\mathrm{H}(14 \mathrm{~B})$ & 106.5 \\
\hline
\end{tabular}




\begin{tabular}{|c|c|c|c|}
\hline $\mathrm{Sc}-\mathrm{C}(14)-\mathrm{H}(14 \mathrm{~B})$ & 106.5 & $\mathrm{Si}(2)-\mathrm{C}(19)-\mathrm{H}(19 \mathrm{~B})$ & 109.5 \\
\hline $\mathrm{H}(14 \mathrm{~A})-\mathrm{C}(14)-\mathrm{H}(14 \mathrm{~B})$ & 106.5 & $\mathrm{H}(19 \mathrm{~A})-\mathrm{C}(19)-\mathrm{H}(19 \mathrm{~B})$ & 109.5 \\
\hline $\mathrm{Si}(1)-\mathrm{C}(15)-\mathrm{H}(15 \mathrm{~A})$ & 109.5 & $\mathrm{Si}(2)-\mathrm{C}(19)-\mathrm{H}(19 \mathrm{C})$ & 109.5 \\
\hline $\mathrm{Si}(1)-\mathrm{C}(15)-\mathrm{H}(15 \mathrm{~B})$ & 109.5 & $\mathrm{H}(19 \mathrm{~A})-\mathrm{C}(19)-\mathrm{H}(19 \mathrm{C})$ & 109.5 \\
\hline $\mathrm{H}(15 \mathrm{~A})-\mathrm{C}(15)-\mathrm{H}(15 \mathrm{~B})$ & 109.5 & $\mathrm{H}(19 \mathrm{~B})-\mathrm{C}(19)-\mathrm{H}(19 \mathrm{C})$ & 109.5 \\
\hline $\mathrm{Si}(1)-\mathrm{C}(15)-\mathrm{H}(15 \mathrm{C})$ & 109.5 & $\mathrm{Si}(2)-\mathrm{C}(20)-\mathrm{H}(20 \mathrm{~A})$ & 109.5 \\
\hline $\mathrm{H}(15 \mathrm{~A})-\mathrm{C}(15)-\mathrm{H}(15 \mathrm{C})$ & 109.5 & $\mathrm{Si}(2)-\mathrm{C}(20)-\mathrm{H}(20 \mathrm{~B})$ & 109.5 \\
\hline $\mathrm{H}(15 \mathrm{~B})-\mathrm{C}(15)-\mathrm{H}(15 \mathrm{C})$ & 109.5 & $\mathrm{H}(20 \mathrm{~A})-\mathrm{C}(20)-\mathrm{H}(20 \mathrm{~B})$ & 109.5 \\
\hline $\mathrm{Si}(1)-\mathrm{C}(16)-\mathrm{H}(16 \mathrm{~A})$ & 109.5 & $\mathrm{Si}(2)-\mathrm{C}(20)-\mathrm{H}(20 \mathrm{C})$ & 109.5 \\
\hline $\mathrm{Si}(1)-\mathrm{C}(16)-\mathrm{H}(16 \mathrm{~B})$ & 109.5 & $\mathrm{H}(20 \mathrm{~A})-\mathrm{C}(20)-\mathrm{H}(20 \mathrm{C})$ & 109.5 \\
\hline$H(16 A)-C(16)-H(16 B)$ & 109.5 & $\mathrm{H}(20 \mathrm{~B})-\mathrm{C}(20)-\mathrm{H}(20 \mathrm{C})$ & 109.5 \\
\hline $\mathrm{Si}(1)-\mathrm{C}(16)-\mathrm{H}(16 \mathrm{C})$ & 109.5 & $\mathrm{Si}(2)-\mathrm{C}(21)-\mathrm{H}(21 \mathrm{~A})$ & 109.5 \\
\hline $\mathrm{H}(16 \mathrm{~A})-\mathrm{C}(16)-\mathrm{H}(16 \mathrm{C})$ & 109.5 & $\mathrm{Si}(2)-\mathrm{C}(21)-\mathrm{H}(21 \mathrm{~B})$ & 109.5 \\
\hline $\mathrm{H}(16 \mathrm{~B})-\mathrm{C}(16)-\mathrm{H}(16 \mathrm{C})$ & 109.5 & $\mathrm{H}(21 \mathrm{~A})-\mathrm{C}(21)-\mathrm{H}(21 \mathrm{~B})$ & 109.5 \\
\hline $\mathrm{Si}(1)-\mathrm{C}(17)-\mathrm{H}(17 \mathrm{~A})$ & 109.5 & $\mathrm{Si}(2)-\mathrm{C}(21)-\mathrm{H}(21 \mathrm{C})$ & 109.5 \\
\hline $\mathrm{Si}(1)-\mathrm{C}(17)-\mathrm{H}(17 \mathrm{~B})$ & 109.5 & $\mathrm{H}(21 \mathrm{~A})-\mathrm{C}(21)-\mathrm{H}(21 \mathrm{C})$ & 109.5 \\
\hline $\mathrm{H}(17 \mathrm{~A})-\mathrm{C}(17)-\mathrm{H}(17 \mathrm{~B})$ & 109.5 & $\mathrm{H}(21 \mathrm{~B})-\mathrm{C}(21)-\mathrm{H}(21 \mathrm{C})$ & 109.5 \\
\hline $\mathrm{Si}(1)-\mathrm{C}(17)-\mathrm{H}(17 \mathrm{C})$ & 109.5 & $\mathrm{O}-\mathrm{C}(22)-\mathrm{C}(23)$ & $105.62(17)$ \\
\hline $\mathrm{H}(17 \mathrm{~A})-\mathrm{C}(17)-\mathrm{H}(17 \mathrm{C})$ & 109.5 & $\mathrm{O}-\mathrm{C}(22)-\mathrm{H}(22 \mathrm{~A})$ & 110.6 \\
\hline $\mathrm{H}(17 \mathrm{~B})-\mathrm{C}(17)-\mathrm{H}(17 \mathrm{C})$ & 109.5 & $\mathrm{C}(23)-\mathrm{C}(22)-\mathrm{H}(22 \mathrm{~A})$ & 110.6 \\
\hline $\mathrm{Si}(2)-\mathrm{C}(18)-\mathrm{Sc}$ & $128.28(10)$ & $\mathrm{O}-\mathrm{C}(22)-\mathrm{H}(22 \mathrm{~B})$ & 110.6 \\
\hline $\mathrm{Si}(2)-\mathrm{C}(18)-\mathrm{H}(18 \mathrm{~A})$ & 105.2 & $\mathrm{C}(23)-\mathrm{C}(22)-\mathrm{H}(22 \mathrm{~B})$ & 110.6 \\
\hline $\mathrm{Sc}-\mathrm{C}(18)-\mathrm{H}(18 \mathrm{~A})$ & 105.2 & $\mathrm{H}(22 \mathrm{~A})-\mathrm{C}(22)-\mathrm{H}(22 \mathrm{~B})$ & 108.7 \\
\hline $\mathrm{Si}(2)-\mathrm{C}(18)-\mathrm{H}(18 \mathrm{~B})$ & 105.2 & $C(22)-C(23)-C(24)$ & $104.73(19)$ \\
\hline $\mathrm{Sc}-\mathrm{C}(18)-\mathrm{H}(18 \mathrm{~B})$ & 105.2 & $\mathrm{C}(22)-\mathrm{C}(23)-\mathrm{H}(23 \mathrm{~A})$ & 110.8 \\
\hline $\mathrm{H}(18 \mathrm{~A})-\mathrm{C}(18)-\mathrm{H}(18 \mathrm{~B})$ & 105.9 & $\mathrm{C}(24)-\mathrm{C}(23)-\mathrm{H}(23 \mathrm{~A})$ & 110.8 \\
\hline $\mathrm{Si}(2)-\mathrm{C}(19)-\mathrm{H}(19 \mathrm{~A})$ & 109.5 & $\mathrm{C}(22)-\mathrm{C}(23)-\mathrm{H}(23 \mathrm{~B})$ & 110.8 \\
\hline
\end{tabular}




\begin{tabular}{lclr}
$\mathrm{C}(24)-\mathrm{C}(23)-\mathrm{H}(23 \mathrm{~B})$ & 110.8 & $\mathrm{H}(24 \mathrm{~A})-\mathrm{C}(24)-\mathrm{H}(24 \mathrm{~B})$ & 108.7 \\
$\mathrm{H}(23 \mathrm{~A})-\mathrm{C}(23)-\mathrm{H}(23 \mathrm{~B})$ & 108.9 & $\mathrm{O}-\mathrm{C}(25)-\mathrm{C}(24)$ & $106.1(2)$ \\
$\mathrm{C}(25)-\mathrm{C}(24)-\mathrm{C}(23)$ & $106.26(19)$ & $\mathrm{O}-\mathrm{C}(25)-\mathrm{H}(25 \mathrm{~A})$ & 110.5 \\
$\mathrm{C}(25)-\mathrm{C}(24)-\mathrm{H}(24 \mathrm{~A})$ & 110.5 & $\mathrm{C}(24)-\mathrm{C}(25)-\mathrm{H}(25 \mathrm{~A})$ & 110.5 \\
$\mathrm{C}(23)-\mathrm{C}(24)-\mathrm{H}(24 \mathrm{~A})$ & 110.5 & $\mathrm{O}-\mathrm{C}(25)-\mathrm{H}(25 \mathrm{~B})$ & 110.5 \\
$\mathrm{C}(25)-\mathrm{C}(24)-\mathrm{H}(24 \mathrm{~B})$ & 110.5 & $\mathrm{C}(24)-\mathrm{C}(25)-\mathrm{H}(25 \mathrm{~B})$ & 110.5 \\
$\mathrm{C}(23)-\mathrm{C}(24)-\mathrm{H}(24 \mathrm{~B})$ & 110.5 & $\mathrm{H}(25 \mathrm{~A})-\mathrm{C}(25)-\mathrm{H}(25 \mathrm{~B})$ & 108.7 \\
& & & \\
\hline
\end{tabular}

\#---End of complex 1b 
Stable 10. Crystal data and structure refinement for $\mathbf{2 a}$

Identification code

Empirical formula

Formula weight

Temperature

Wavelength

Crystal system

space group

Unit cell dimensions

Volume

Z, Calculated density

Absorption coefficient

$\mathrm{F}(000)$

Crystal size

Theta range for data collection

Limiting indices

Reflections collected / unique

Completeness to theta $=26.00$

Absorption correction

Max. and min. transmission

Refinement method

Data / restraints / parameters

Goodness-of-fit on $\mathrm{F}^{\wedge} 2$

Final $\mathrm{R}$ indices $[\mathrm{I}>2 \operatorname{sigma}(\mathrm{I})]$ q293m

C42 H59 N4 O Si Lu

838.99

$187(2) \mathrm{K}$

$0.71073 \AA$

Triclinic

P-1

$\mathrm{a}=10.3754(6) \AA \quad$ alpha $=88.3420(10) \mathrm{deg}$.

$\mathrm{b}=19.2503(11) \AA \quad$ beta $=89.6590(10) \mathrm{deg}$.

$\mathrm{c}=20.9445(12) \AA \quad$ gamma $=88.0490(10) \mathrm{deg}$.

$4179.0(4) \AA^{3}$

4, $\quad 1.334 \mathrm{Mg} / \mathrm{m}^{3}$

$2.426 \mathrm{~mm}^{-1}$

1728

$0.18 \times 0.13 \times 0.11 \mathrm{~mm}$

1.42 to $26.00 \mathrm{deg}$.

$-12<=\mathrm{h}<=12,-18<=\mathrm{k}<=23,-25<=\mathrm{l}<=19$

$23450 / 16002[\mathrm{R}(\mathrm{int})=0.0415]$

$97.3 \%$

Semi-empirical from equivalents

0.7762 and 0.6693

Full-matrix least-squares on $\mathrm{F}^{2}$

16002 / 0 / 905

0.870

$\mathrm{R} 1=0.0405, \mathrm{wR} 2=0.0791$ 
$\mathrm{R}$ indices (all data)

Largest diff. peak and hole
$\mathrm{R} 1=0.0659, \mathrm{wR} 2=0.0860$

1.775 and $-0.882 \mathrm{e}^{-3}$ 
Stable 11. Atomic coordinates ( $\left.\begin{array}{ll}x & 10^{4}\end{array}\right)$ and equivalent isotropic displacement parameters $\left(\AA^{2} \times 10^{3}\right)$ for $2 a$. $U(e q)$ is defined as one third of the trace of the orthogonalized Uij tensor.

\begin{tabular}{|c|c|c|c|c|}
\hline & $\mathrm{x}$ & $\mathrm{y}$ & $\mathrm{z}$ & $\mathrm{U}(\mathrm{eq})$ \\
\hline $\mathrm{Lu}(1)$ & 9192(1) & $7186(1)$ & $5987(1)$ & $29(1)$ \\
\hline $\mathrm{Lu}(2)$ & $5540(1)$ & $7176(1)$ & $989(1)$ & $30(1)$ \\
\hline $\operatorname{Si}(1)$ & $12229(2)$ & $6082(1)$ & $6462(1)$ & $61(1)$ \\
\hline $\operatorname{Si}(5)$ & $2617(2)$ & $6085(1)$ & 1481(1) & $58(1)$ \\
\hline $\mathrm{O}(1)$ & $9592(3)$ & $6955(2)$ & $4925(2)$ & $37(1)$ \\
\hline $\mathrm{O}(2)$ & 5191(3) & $6924(2)$ & $-66(2)$ & $37(1)$ \\
\hline $\mathrm{N}(1)$ & $8137(4)$ & $6164(2)$ & $5973(2)$ & $33(1)$ \\
\hline $\mathrm{N}(2)$ & $8114(4)$ & $7058(2)$ & $7019(2)$ & $31(1)$ \\
\hline $\mathrm{N}(3)$ & $7294(4)$ & $7716(2)$ & $5596(2)$ & $33(1)$ \\
\hline $\mathrm{N}(4)$ & $9527(4)$ & $8417(2)$ & $5780(2)$ & $30(1)$ \\
\hline $\mathrm{N}(5)$ & $6713(4)$ & $6153(2)$ & $995(2)$ & $33(1)$ \\
\hline $\mathrm{N}(6)$ & $6611(4)$ & $7083(2)$ & $2019(2)$ & $29(1)$ \\
\hline $\mathrm{N}(7)$ & $7409(4)$ & $7687(2)$ & $587(2)$ & $35(1)$ \\
\hline $\mathrm{N}(8)$ & $5086(4)$ & $8399(2)$ & $748(2)$ & $33(1)$ \\
\hline$C(1)$ & $7866(5)$ & $5642(3)$ & $5581(3)$ & $43(2)$ \\
\hline $\mathrm{C}(2)$ & 7081(6) & $5166(3)$ & $5883(3)$ & $52(2)$ \\
\hline $\mathrm{C}(3)$ & $6836(6)$ & $5403(3)$ & $6484(3)$ & $54(2)$ \\
\hline$C(4)$ & $7473(5)$ & $6019(3)$ & $6528(3)$ & $36(1)$ \\
\hline$C(5)$ & $7495(5)$ & $6484(3)$ & $7044(2)$ & $38(1)$ \\
\hline$C(6)$ & $8048(5)$ & $7479(3)$ & $7586(2)$ & $33(1)$ \\
\hline
\end{tabular}




\begin{tabular}{|c|c|c|c|c|}
\hline$C(7)$ & $8898(5)$ & $7323(3)$ & $8092(3)$ & $36(1)$ \\
\hline$C(8)$ & $8835(5)$ & $7749(3)$ & $8614(3)$ & $44(2)$ \\
\hline$C(9)$ & $7971(5)$ & $8312(3)$ & $8638(3)$ & $44(2)$ \\
\hline$C(10)$ & $7128(5)$ & $8449(3)$ & $8141(3)$ & $41(1)$ \\
\hline$C(11)$ & $7130(5)$ & $8033(3)$ & $7611(3)$ & $37(1)$ \\
\hline$C(12)$ & $6118(5)$ & $8179(3)$ & $7090(3)$ & $46(2)$ \\
\hline$C(13)$ & $4936(6)$ & $7738(4)$ & $7255(4)$ & $82(2)$ \\
\hline$C(14)$ & $5716(6)$ & $8915(3)$ & $6990(3)$ & $66(2)$ \\
\hline$C(15)$ & $9794(5)$ & $6693(3)$ & $8099(3)$ & $45(2)$ \\
\hline$C(16)$ & $11109(6)$ & $6813(3)$ & $8376(3)$ & $63(2)$ \\
\hline$C(17)$ & $9207(6)$ & $6089(3)$ & $8475(3)$ & $66(2)$ \\
\hline $\mathrm{C}(18)$ & $6049(5)$ & $7559(3)$ & $5504(3)$ & $42(1)$ \\
\hline$C(19)$ & $5287(5)$ & $8144(3)$ & $5358(3)$ & $48(2)$ \\
\hline$C(20)$ & $6081(5)$ & $8697(3)$ & $5340(3)$ & $45(2)$ \\
\hline $\mathrm{C}(21)$ & $7319(5)$ & $8424(3)$ & $5493(2)$ & $34(1)$ \\
\hline $\mathrm{C}(22)$ & $8504(5)$ & $8757(3)$ & $5580(2)$ & $34(1)$ \\
\hline $\mathrm{C}(23)$ & $10686(5)$ & $8811(3)$ & $5805(3)$ & $31(1)$ \\
\hline$C(24)$ & $11364(5)$ & $8956(3)$ & $5232(3)$ & $33(1)$ \\
\hline$C(25)$ & $12433(5)$ & $9369(3)$ & $5263(3)$ & $39(1)$ \\
\hline$C(26)$ & $12835(5)$ & $9618(3)$ & $5838(3)$ & $40(1)$ \\
\hline $\mathrm{C}(27)$ & $12192(5)$ & $9445(3)$ & $6396(3)$ & $39(1)$ \\
\hline $\mathrm{C}(28)$ & $11118(5)$ & $9039(3)$ & $6394(2)$ & $32(1)$ \\
\hline $\mathrm{C}(29)$ & $10404(5)$ & $8872(3)$ & $7011(2)$ & $39(1)$ \\
\hline $\mathrm{C}(30)$ & $9413(6)$ & $9444(3)$ & $7160(3)$ & $60(2)$ \\
\hline $\mathrm{C}(31)$ & $11298(6)$ & $8737(4)$ & $7573(3)$ & $68(2)$ \\
\hline$C(32)$ & $10974(5)$ & $8664(3)$ & $4606(2)$ & $37(1)$ \\
\hline $\mathrm{C}(33)$ & $12096(6)$ & $8263(4)$ & $4284(3)$ & $67(2)$ \\
\hline
\end{tabular}




\begin{tabular}{|c|c|c|c|c|}
\hline$C(34)$ & $10415(7)$ & $9216(3)$ & $4147(3)$ & $74(2)$ \\
\hline$C(35)$ & $11314(6)$ & $6951(3)$ & $6328(3)$ & $50(2)$ \\
\hline$C(36)$ & $13166(8)$ & $5832(5)$ & $5737(4)$ & $117(4)$ \\
\hline$C(37)$ & $11045(7)$ & $5393(3)$ & $6655(4)$ & $94(3)$ \\
\hline$C(38)$ & $13398(6)$ & $6005(4)$ & $7131(3)$ & $78(2)$ \\
\hline C(39) & $8699(5)$ & $7110(3)$ & $4399(3)$ & $49(2)$ \\
\hline$C(40)$ & $9257(9)$ & $6753(6)$ & $3844(4)$ & $131(5)$ \\
\hline$C(41)$ & 10283(9) & $6364(5)$ & $4023(4)$ & $116(4)$ \\
\hline$C(42)$ & $10588(5)$ & $6479(3)$ & $4701(3)$ & $48(2)$ \\
\hline$C(43)$ & $7051(5)$ & $5618(3)$ & $613(3)$ & $39(1)$ \\
\hline$C(44)$ & $7916(6)$ & $5162(3)$ & $913(3)$ & $51(2)$ \\
\hline$C(45)$ & $8155(6)$ & $5419(3)$ & 1511(3) & $54(2)$ \\
\hline$C(46)$ & $7412(5)$ & $6032(3)$ & $1547(3)$ & $37(1)$ \\
\hline$C(47)$ & $7315(5)$ & $6513(3)$ & $2045(2)$ & $36(1)$ \\
\hline$C(48)$ & $6602(5)$ & $7498(3)$ & $2590(2)$ & $31(1)$ \\
\hline$C(49)$ & $5809(5)$ & $7310(3)$ & $3105(2)$ & $34(1)$ \\
\hline$C(50)$ & $5806(5)$ & $7730(3)$ & $3637(3)$ & $43(2)$ \\
\hline$C(51)$ & $6536(6)$ & $8303(3)$ & $3665(3)$ & $45(2)$ \\
\hline$C(52)$ & $7309(6)$ & $8474(3)$ & $3158(3)$ & $46(2)$ \\
\hline$C(53)$ & $7373(5)$ & $8080(3)$ & $2607(3)$ & $37(1)$ \\
\hline$C(54)$ & $8316(6)$ & $8260(3)$ & $2079(3)$ & $52(2)$ \\
\hline$C(55)$ & $8486(8)$ & $9044(4)$ & $1970(3)$ & $94(3)$ \\
\hline$C(56)$ & $9608(6)$ & $7896(5)$ & $2196(4)$ & $103(3)$ \\
\hline$C(57)$ & $5041(5)$ & $6654(3)$ & $3125(3)$ & $45(2)$ \\
\hline$C(58)$ & $3683(6)$ & $6754(3)$ & $3389(3)$ & $61(2)$ \\
\hline $\mathrm{C}(59)$ & $5746(6)$ & $6072(3)$ & $3506(3)$ & $68(2)$ \\
\hline$C(60)$ & $8679(5)$ & $7508(3)$ & $520(3)$ & $45(2)$ \\
\hline
\end{tabular}




\begin{tabular}{|c|c|c|c|c|}
\hline $\mathrm{C}(61)$ & $9378(6)$ & $8082(3)$ & $350(3)$ & $50(2)$ \\
\hline$C(62)$ & $8523(5)$ & $8642(3)$ & $303(3)$ & $45(2)$ \\
\hline$C(63)$ & 7301(5) & $8383(3)$ & $457(2)$ & $35(1)$ \\
\hline C(64) & 6093(5) & $8722(3)$ & $528(2)$ & $36(1)$ \\
\hline $\mathrm{C}(65)$ & $3888(5)$ & $8796(3)$ & $751(3)$ & $35(1)$ \\
\hline C(66) & $3242(5)$ & $8960(3)$ & $181(3)$ & $38(1)$ \\
\hline $\mathrm{C}(67)$ & $2108(6)$ & $9367(3)$ & 204(3) & $48(2)$ \\
\hline $\mathrm{C}(68)$ & $1610(6)$ & $9595(3)$ & $775(3)$ & $54(2)$ \\
\hline C(69) & $2239(6)$ & $9409(3)$ & $1337(3)$ & $50(2)$ \\
\hline $\mathrm{C}(70)$ & $3358(5)$ & $9002(3)$ & 1343(3) & $38(1)$ \\
\hline$C(71)$ & $4053(5)$ & $8826(3)$ & 1970(3) & $44(2)$ \\
\hline$C(72)$ & $3133(6)$ & $8608(4)$ & $2500(3)$ & $74(2)$ \\
\hline$C(73)$ & $4814(8)$ & $9436(4)$ & $2168(4)$ & $93(3)$ \\
\hline$C(74)$ & $3702(6)$ & $8694(3)$ & $-459(3)$ & $44(2)$ \\
\hline$C(75)$ & $2676(6)$ & $8274(4)$ & $-776(3)$ & $71(2)$ \\
\hline$C(76)$ & 4113(6) & $9276(3)$ & $-905(3)$ & $64(2)$ \\
\hline $\mathrm{C}(77)$ & $3408(6)$ & $6942(3)$ & $1332(3)$ & $48(2)$ \\
\hline C(78) & $3852(6)$ & $5408(3)$ & $1720(3)$ & $72(2)$ \\
\hline C(79) & $1768(7)$ & $5776(4)$ & $756(3)$ & $98(3)$ \\
\hline $\mathrm{C}(80)$ & $1382(6)$ & $6056(4)$ & $2138(3)$ & $85(3)$ \\
\hline $\mathrm{C}(81)$ & $6095(6)$ & $7038(3)$ & $-588(3)$ & $51(2)$ \\
\hline $\mathrm{C}(82)$ & $5648(8)$ & $6615(5)$ & $-1114(3)$ & $115(4)$ \\
\hline $\mathrm{C}(83)$ & $4512(8)$ & $6351(5)$ & $-976(4)$ & $117(4)$ \\
\hline C(84) & $4226(5)$ & $6453(3)$ & $-281(3)$ & $52(2)$ \\
\hline
\end{tabular}


Stable 12. Anisotropic displacement parameters $\left(\AA^{2} x 1^{3}\right)$ for $2 a$. The anisotropic displacement factor exponent takes the form: $-2 \pi^{2}\left[h^{2} a * 2 U^{11}+\ldots\right.$ $\left.+2 \mathrm{~h} \mathrm{k} \mathrm{a*} \mathbf{b}^{*} \mathbf{U}^{12}\right]$

\begin{tabular}{|c|c|c|c|c|c|c|}
\hline & U11 & U22 & U33 & $\mathrm{U} 23$ & U13 & U12 \\
\hline $\mathrm{Lu}(1)$ & $32(1)$ & $28(1)$ & $27(1)$ & $-3(1)$ & $2(1)$ & $-3(1)$ \\
\hline $\mathrm{Lu}(2)$ & $35(1)$ & $27(1)$ & $27(1)$ & $-2(1)$ & $3(1)$ & $-1(1)$ \\
\hline $\operatorname{Si}(1)$ & $64(1)$ & $60(1)$ & $58(1)$ & $-15(1)$ & $1(1)$ & $-2(1)$ \\
\hline $\operatorname{Si}(5)$ & $62(1)$ & $62(1)$ & $51(1)$ & $-14(1)$ & $9(1)$ & $-9(1)$ \\
\hline $\mathrm{O}(1)$ & $39(2)$ & $41(2)$ & $31(2)$ & $-10(2)$ & $1(2)$ & $2(2)$ \\
\hline $\mathrm{O}(2)$ & $42(2)$ & $43(2)$ & $27(2)$ & $-6(2)$ & $5(2)$ & $-11(2)$ \\
\hline $\mathrm{N}(1)$ & $36(2)$ & $33(3)$ & $31(3)$ & $-8(2)$ & $3(2)$ & $-3(2)$ \\
\hline $\mathrm{N}(2)$ & $30(2)$ & $37(3)$ & $27(3)$ & $-6(2)$ & $1(2)$ & $-1(2)$ \\
\hline $\mathrm{N}(3)$ & $33(2)$ & $35(3)$ & $31(3)$ & $-6(2)$ & $-1(2)$ & $-7(2)$ \\
\hline $\mathrm{N}(4)$ & $29(2)$ & $31(3)$ & $29(3)$ & $-2(2)$ & $0(2)$ & $-2(2)$ \\
\hline $\mathrm{N}(5)$ & $35(2)$ & $30(3)$ & $35(3)$ & $-4(2)$ & $-3(2)$ & $0(2)$ \\
\hline $\mathrm{N}(6)$ & $37(2)$ & $27(2)$ & $24(2)$ & $-5(2)$ & $5(2)$ & $-3(2)$ \\
\hline $\mathrm{N}(7)$ & $41(3)$ & $36(3)$ & $29(3)$ & $-2(2)$ & $5(2)$ & $-4(2)$ \\
\hline $\mathrm{N}(8)$ & $38(3)$ & $35(3)$ & $26(3)$ & $-4(2)$ & $2(2)$ & $-1(2)$ \\
\hline $\mathrm{C}(1)$ & $51(4)$ & $44(4)$ & $36(4)$ & $-13(3)$ & $8(3)$ & $-7(3)$ \\
\hline $\mathrm{C}(2)$ & $65(4)$ & $40(4)$ & $52(4)$ & $-22(3)$ & $15(3)$ & $-23(3)$ \\
\hline$C(3)$ & $62(4)$ & $53(4)$ & $49(4)$ & $-11(3)$ & $21(3)$ & $-31(3)$ \\
\hline $\mathrm{C}(4)$ & $41(3)$ & $35(3)$ & $33(3)$ & $-7(3)$ & $7(3)$ & $-6(3)$ \\
\hline$C(5)$ & $38(3)$ & $48(4)$ & $27(3)$ & $-2(3)$ & $7(2)$ & $-9(3)$ \\
\hline$C(6)$ & $37(3)$ & $37(3)$ & $26(3)$ & $-6(2)$ & $10(2)$ & $-14(3)$ \\
\hline
\end{tabular}




\begin{tabular}{|c|c|c|c|c|c|c|}
\hline$C(7)$ & $38(3)$ & $36(3)$ & $34(3)$ & $-7(3)$ & $8(3)$ & $-5(3)$ \\
\hline$C(8)$ & $54(4)$ & $49(4)$ & $30(3)$ & $-4(3)$ & $2(3)$ & $-10(3)$ \\
\hline$C(9)$ & $55(4)$ & $43(4)$ & $35(4)$ & $-13(3)$ & $16(3)$ & $-10(3)$ \\
\hline$C(10)$ & $52(4)$ & $36(3)$ & $34(3)$ & $-5(3)$ & $13(3)$ & $1(3)$ \\
\hline$C(11)$ & $45(3)$ & $35(3)$ & $31(3)$ & $-4(3)$ & $9(3)$ & $-1(3)$ \\
\hline$C(12)$ & $41(3)$ & $63(4)$ & $32(3)$ & $-13(3)$ & $1(3)$ & $18(3)$ \\
\hline$C(13)$ & $59(5)$ & $80(6)$ & $108(7)$ & $-7(5)$ & $-15(4)$ & $-2(4)$ \\
\hline$C(14)$ & $70(5)$ & $77(5)$ & $49(4)$ & $-8(4)$ & $-5(4)$ & $19(4)$ \\
\hline$C(15)$ & $40(3)$ & $54(4)$ & $40(4)$ & $-8(3)$ & $-4(3)$ & $2(3)$ \\
\hline$C(16)$ & $60(4)$ & $72(5)$ & $58(5)$ & $-6(4)$ & $-11(4)$ & $7(4)$ \\
\hline$C(17)$ & $60(4)$ & $46(4)$ & $92(6)$ & $5(4)$ & $3(4)$ & $11(3)$ \\
\hline$C(18)$ & $41(3)$ & $45(4)$ & $41(4)$ & $-6(3)$ & $1(3)$ & $-12(3)$ \\
\hline$C(19)$ & $34(3)$ & $70(5)$ & $41(4)$ & $-5(3)$ & $-2(3)$ & $-3(3)$ \\
\hline$C(20)$ & $39(3)$ & $58(4)$ & $37(4)$ & $-2(3)$ & $-5(3)$ & $10(3)$ \\
\hline$C(21)$ & $36(3)$ & $46(4)$ & $21(3)$ & $-4(3)$ & $3(2)$ & $-1(3)$ \\
\hline$C(22)$ & $37(3)$ & $36(3)$ & $28(3)$ & $-3(2)$ & $6(2)$ & $-2(3)$ \\
\hline$C(23)$ & $31(3)$ & $24(3)$ & $38(3)$ & $3(2)$ & $-1(2)$ & $3(2)$ \\
\hline$C(24)$ & $32(3)$ & $24(3)$ & $41(3)$ & $0(2)$ & $0(3)$ & $3(2)$ \\
\hline$C(25)$ & $41(3)$ & $32(3)$ & $43(4)$ & $0(3)$ & $10(3)$ & $0(3)$ \\
\hline$C(26)$ & $32(3)$ & $38(3)$ & $49(4)$ & $0(3)$ & $-1(3)$ & $-9(3)$ \\
\hline$C(27)$ & $41(3)$ & $37(3)$ & $38(4)$ & $-5(3)$ & $-6(3)$ & $-3(3)$ \\
\hline$C(28)$ & $36(3)$ & $26(3)$ & $34(3)$ & $-2(2)$ & $-2(2)$ & $-1(2)$ \\
\hline$C(29)$ & $52(4)$ & $38(3)$ & $28(3)$ & $1(3)$ & $0(3)$ & $-15(3)$ \\
\hline$C(30)$ & $77(5)$ & $57(4)$ & $46(4)$ & $-1(3)$ & $22(3)$ & $-14(4)$ \\
\hline$C(31)$ & $71(5)$ & $96(6)$ & $38(4)$ & $18(4)$ & $-6(3)$ & $-24(4)$ \\
\hline$C(32)$ & $39(3)$ & $37(3)$ & $35(3)$ & $-6(3)$ & $4(3)$ & $-4(3)$ \\
\hline$C(33)$ & $67(5)$ & $87(6)$ & $47(4)$ & $-29(4)$ & $7(3)$ & $6(4)$ \\
\hline
\end{tabular}




\begin{tabular}{|c|c|c|c|c|c|c|}
\hline$C(34)$ & $103(6)$ & $66(5)$ & $51(5)$ & $-4(4)$ & $-30(4)$ & $17(4)$ \\
\hline$C(35)$ & $87(5)$ & $29(3)$ & $35(4)$ & $-12(3)$ & $18(3)$ & $-8(3)$ \\
\hline$C(36)$ & $123(7)$ & $158(9)$ & $67(6)$ & $-30(6)$ & $-4(5)$ & $74(7)$ \\
\hline$C(37)$ & $79(5)$ & $37(4)$ & 164(9) & $-1(5)$ & $-37(5)$ & $15(4)$ \\
\hline$C(38)$ & $80(5)$ & $74(6)$ & $80(6)$ & $-12(4)$ & $0(4)$ & $-7(4)$ \\
\hline$C(39)$ & $41(3)$ & $70(5)$ & $35(4)$ & $-5(3)$ & $-12(3)$ & $4(3)$ \\
\hline$C(40)$ & 121(8) & $235(12)$ & $35(5)$ & $-59(6)$ & $-30(5)$ & $98(8)$ \\
\hline$C(41)$ & $123(8)$ & $176(10)$ & $49(5)$ & $-67(6)$ & $-11(5)$ & $64(7)$ \\
\hline$C(42)$ & $44(4)$ & $56(4)$ & $45(4)$ & $-9(3)$ & $10(3)$ & $6(3)$ \\
\hline$C(43)$ & $51(4)$ & $34(3)$ & $31(3)$ & $-10(3)$ & $1(3)$ & $4(3)$ \\
\hline$C(44)$ & $60(4)$ & $37(4)$ & $55(4)$ & $-17(3)$ & $-6(3)$ & $19(3)$ \\
\hline$C(45)$ & $62(4)$ & $51(4)$ & $46(4)$ & $-8(3)$ & $-20(3)$ & $23(3)$ \\
\hline$C(46)$ & $38(3)$ & $34(3)$ & $39(4)$ & $-5(3)$ & $-1(3)$ & $0(3)$ \\
\hline$C(47)$ & $37(3)$ & $44(4)$ & $28(3)$ & $-5(3)$ & $-3(2)$ & $-2(3)$ \\
\hline$C(48)$ & $40(3)$ & $31(3)$ & $22(3)$ & $-5(2)$ & $-4(2)$ & $1(2)$ \\
\hline$C(49)$ & $39(3)$ & $34(3)$ & $28(3)$ & $-6(2)$ & $-2(2)$ & $3(3)$ \\
\hline$C(50)$ & $52(4)$ & $50(4)$ & $27(3)$ & $-5(3)$ & $2(3)$ & $1(3)$ \\
\hline$C(51)$ & $59(4)$ & $45(4)$ & $31(3)$ & $-11(3)$ & $-7(3)$ & $3(3)$ \\
\hline$C(52)$ & $63(4)$ & $38(4)$ & $39(4)$ & $-8(3)$ & $-7(3)$ & $-10(3)$ \\
\hline$C(53)$ & $51(4)$ & $33(3)$ & $29(3)$ & $-6(3)$ & $-7(3)$ & $-4(3)$ \\
\hline$C(54)$ & $69(4)$ & $60(4)$ & $30(4)$ & $-7(3)$ & $1(3)$ & $-26(4)$ \\
\hline$C(55)$ & $148(8)$ & $78(6)$ & $60(5)$ & $-7(4)$ & $24(5)$ & $-64(5)$ \\
\hline$C(56)$ & $51(5)$ & $177(10)$ & $81(6)$ & $-1(6)$ & $12(4)$ & $-10(5)$ \\
\hline$C(57)$ & $52(4)$ & $46(4)$ & $38(4)$ & $-11(3)$ & $10(3)$ & $-9(3)$ \\
\hline $\mathrm{C}(58)$ & $58(4)$ & $65(5)$ & $59(5)$ & $1(4)$ & $13(3)$ & $-7(3)$ \\
\hline$C(59)$ & $63(4)$ & $43(4)$ & $99(6)$ & $6(4)$ & $6(4)$ & $-11(3)$ \\
\hline$C(60)$ & $38(3)$ & $56(4)$ & $40(4)$ & $-6(3)$ & $5(3)$ & $5(3)$ \\
\hline
\end{tabular}




\begin{tabular}{|c|c|c|c|c|c|c|}
\hline$C(61)$ & $37(3)$ & 71(5) & $42(4)$ & $-8(3)$ & $9(3)$ & $-14(3)$ \\
\hline$C(62)$ & $47(4)$ & $54(4)$ & $35(4)$ & $-1(3)$ & 6(3) & $-16(3)$ \\
\hline$C(63)$ & $39(3)$ & $40(4)$ & $27(3)$ & $-2(3)$ & 4(3) & $-7(3)$ \\
\hline $\mathrm{C}(64)$ & $52(4)$ & $31(3)$ & $25(3)$ & $-4(2)$ & 1(3) & 1(3) \\
\hline$C(65)$ & $42(3)$ & $23(3)$ & $40(4)$ & $-2(3)$ & 1(3) & $-1(2)$ \\
\hline$C(66)$ & $46(3)$ & $30(3)$ & $38(4)$ & $0(3)$ & $7(3)$ & $1(3)$ \\
\hline$C(67)$ & $61(4)$ & $44(4)$ & $39(4)$ & $-1(3)$ & $5(3)$ & $9(3)$ \\
\hline $\mathrm{C}(68)$ & $59(4)$ & $51(4)$ & $51(4)$ & 2(3) & $6(3)$ & $18(3)$ \\
\hline $\mathrm{C}(69)$ & $59(4)$ & $47(4)$ & $42(4)$ & $-5(3)$ & $17(3)$ & $15(3)$ \\
\hline$C(70)$ & $46(3)$ & $30(3)$ & $37(3)$ & $0(3)$ & $7(3)$ & $-2(3)$ \\
\hline $\mathrm{C}(71)$ & $54(4)$ & $37(4)$ & $39(4)$ & $-3(3)$ & $-1(3)$ & $8(3)$ \\
\hline$C(72)$ & $71(5)$ & $103(6)$ & $45(4)$ & $22(4)$ & $17(4)$ & $11(4)$ \\
\hline$C(73)$ & $121(7)$ & $84(6)$ & $75(6)$ & $12(5)$ & $-31(5)$ & $-31(5)$ \\
\hline$C(74)$ & $55(4)$ & $39(4)$ & $37(4)$ & $-1(3)$ & $-1(3)$ & $15(3)$ \\
\hline$C(75)$ & $86(5)$ & $77(5)$ & $49(5)$ & $-22(4)$ & $-4(4)$ & $-2(4)$ \\
\hline$C(76)$ & $92(5)$ & $60(5)$ & $39(4)$ & $-1(3)$ & $20(4)$ & $6(4)$ \\
\hline $\mathrm{C}(77)$ & $75(4)$ & $27(3)$ & $43(4)$ & $-13(3)$ & $-4(3)$ & 1(3) \\
\hline $\mathrm{C}(78)$ & $80(5)$ & $47(4)$ & $90(6)$ & $-4(4)$ & $22(4)$ & $-10(4)$ \\
\hline $\mathrm{C}(79)$ & $115(7)$ & $129(8)$ & $55(5)$ & $-22(5)$ & $12(5)$ & $-62(6)$ \\
\hline $\mathrm{C}(80)$ & $69(5)$ & $125(7)$ & $64(5)$ & $-19(5)$ & $16(4)$ & $-10(5)$ \\
\hline$C(81)$ & $60(4)$ & $67(5)$ & $27(3)$ & $-5(3)$ & $17(3)$ & $-13(3)$ \\
\hline $\mathrm{C}(82)$ & $104(7)$ & $199(11)$ & $48(5)$ & $-68(6)$ & $33(5)$ & $-75(7)$ \\
\hline$C(83)$ & $107(7)$ & 191(11) & $62(6)$ & $-78(6)$ & $26(5)$ & $-83(7)$ \\
\hline $\mathrm{C}(84)$ & $45(4)$ & $58(4)$ & $55(4)$ & $-9(3)$ & 2(3) & $-14(3)$ \\
\hline
\end{tabular}


Stable 13. Bond lengths $[\AA ̊]$ and angles $\left[{ }^{\circ}\right]$ for $2 a$.

\begin{tabular}{|c|c|c|c|}
\hline $\mathrm{Lu}(1)-\mathrm{N}(1)$ & $2.286(4)$ & $\mathrm{N}(1)-\mathrm{C}(4)$ & $1.374(6)$ \\
\hline $\mathrm{Lu}(1)-\mathrm{O}(1)$ & $2.314(3)$ & $\mathrm{N}(2)-\mathrm{C}(5)$ & $1.297(6)$ \\
\hline $\mathrm{Lu}(1)-\mathrm{N}(3)$ & $2.329(4)$ & $\mathrm{N}(2)-\mathrm{C}(6)$ & $1.457(6)$ \\
\hline $\mathrm{Lu}(1)-\mathrm{C}(35)$ & $2.344(6)$ & $\mathrm{N}(3)-\mathrm{C}(18)$ & $1.353(6)$ \\
\hline $\mathrm{Lu}(1)-\mathrm{N}(4)$ & $2.433(4)$ & $\mathrm{N}(3)-\mathrm{C}(21)$ & $1.375(7)$ \\
\hline $\mathrm{Lu}(1)-\mathrm{N}(2)$ & $2.437(4)$ & $\mathrm{N}(4)-\mathrm{C}(22)$ & $1.294(6)$ \\
\hline $\mathrm{Lu}(2)-\mathrm{N}(5)$ & $2.279(4)$ & $\mathrm{N}(4)-\mathrm{C}(23)$ & $1.445(6)$ \\
\hline $\mathrm{Lu}(2)-\mathrm{O}(2)$ & $2.309(3)$ & $\mathrm{N}(5)-\mathrm{C}(43)$ & $1.357(6)$ \\
\hline $\mathrm{Lu}(2)-\mathrm{N}(7)$ & $2.347(4)$ & $\mathrm{N}(5)-\mathrm{C}(46)$ & $1.379(6)$ \\
\hline $\mathrm{Lu}(2)-\mathrm{C}(77)$ & $2.375(6)$ & $\mathrm{N}(6)-\mathrm{C}(47)$ & $1.298(6)$ \\
\hline $\mathrm{Lu}(2)-\mathrm{N}(8)$ & $2.426(4)$ & $\mathrm{N}(6)-\mathrm{C}(48)$ & $1.457(6)$ \\
\hline $\mathrm{Lu}(2)-\mathrm{N}(6)$ & $2.431(4)$ & $\mathrm{N}(7)-\mathrm{C}(60)$ & $1.357(6)$ \\
\hline $\mathrm{Si}(1)-\mathrm{C}(38)$ & $1.858(7)$ & $\mathrm{N}(7)-\mathrm{C}(63)$ & $1.361(7)$ \\
\hline $\mathrm{Si}(1)-\mathrm{C}(36)$ & $1.864(7)$ & $\mathrm{N}(8)-\mathrm{C}(64)$ & $1.307(6)$ \\
\hline $\mathrm{Si}(1)-\mathrm{C}(37)$ & $1.872(7)$ & $\mathrm{N}(8)-\mathrm{C}(65)$ & $1.437(6)$ \\
\hline $\mathrm{Si}(1)-\mathrm{C}(35)$ & $1.909(6)$ & $\mathrm{C}(1)-\mathrm{C}(2)$ & $1.384(8)$ \\
\hline $\mathrm{Si}(5)-\mathrm{C}(78)$ & $1.856(7)$ & $\mathrm{C}(1)-\mathrm{H}(1)$ & 0.9300 \\
\hline $\mathrm{Si}(5)-\mathrm{C}(80)$ & $1.875(7)$ & $\mathrm{C}(2)-\mathrm{C}(3)$ & $1.371(7)$ \\
\hline $\operatorname{Si}(5)-C(79)$ & $1.882(6)$ & $\mathrm{C}(2)-\mathrm{H}(2)$ & 0.9300 \\
\hline $\operatorname{Si}(5)-C(77)$ & $1.885(6)$ & $C(3)-C(4)$ & $1.381(7)$ \\
\hline $\mathrm{O}(1)-\mathrm{C}(42)$ & $1.442(6)$ & $\mathrm{C}(3)-\mathrm{H}(3)$ & 0.9300 \\
\hline $\mathrm{O}(1)-\mathrm{C}(39)$ & $1.461(6)$ & $C(4)-C(5)$ & $1.423(7)$ \\
\hline $\mathrm{O}(2)-\mathrm{C}(81)$ & $1.454(6)$ & $\mathrm{C}(5)-\mathrm{H}(5)$ & 0.9300 \\
\hline $\mathrm{O}(2)-\mathrm{C}(84)$ & $1.455(6)$ & $C(6)-C(7)$ & $1.402(7)$ \\
\hline $\mathrm{N}(1)-\mathrm{C}(1)$ & $1.353(6)$ & $\mathrm{C}(6)-\mathrm{C}(11)$ & $1.408(7)$ \\
\hline
\end{tabular}




\begin{tabular}{|c|c|c|c|}
\hline$C(7)-C(8)$ & $1.387(7)$ & $\mathrm{C}(18)-\mathrm{C}(19)$ & $1.382(8)$ \\
\hline$C(7)-C(15)$ & $1.502(7)$ & $\mathrm{C}(18)-\mathrm{H}(18)$ & 0.9300 \\
\hline $\mathrm{C}(8)-\mathrm{C}(9)$ & $1.384(7)$ & $C(19)-C(20)$ & $1.368(8)$ \\
\hline $\mathrm{C}(8)-\mathrm{H}(8)$ & 0.9300 & $\mathrm{C}(19)-\mathrm{H}(19)$ & 0.9300 \\
\hline$C(9)-C(10)$ & $1.378(7)$ & $\mathrm{C}(20)-\mathrm{C}(21)$ & $1.407(7)$ \\
\hline $\mathrm{C}(9)-\mathrm{H}(9)$ & 0.9300 & $\mathrm{C}(20)-\mathrm{H}(20)$ & 0.9300 \\
\hline$C(10)-C(11)$ & $1.386(7)$ & $\mathrm{C}(21)-\mathrm{C}(22)$ & $1.421(7)$ \\
\hline $\mathrm{C}(10)-\mathrm{H}(10)$ & 0.9300 & $\mathrm{C}(22)-\mathrm{H}(22)$ & 0.9300 \\
\hline$C(11)-C(12)$ & $1.531(7)$ & $\mathrm{C}(23)-\mathrm{C}(28)$ & $1.402(7)$ \\
\hline$C(12)-C(14)$ & $1.472(8)$ & $C(23)-C(24)$ & $1.412(7)$ \\
\hline$C(12)-C(13)$ & $1.548(9)$ & $C(24)-C(25)$ & $1.390(7)$ \\
\hline $\mathrm{C}(12)-\mathrm{H}(12)$ & 0.9800 & $C(24)-C(32)$ & $1.506(7)$ \\
\hline $\mathrm{C}(13)-\mathrm{H}(13 \mathrm{~A})$ & 0.9600 & $C(25)-C(26)$ & $1.381(7)$ \\
\hline $\mathrm{C}(13)-\mathrm{H}(13 \mathrm{~B})$ & 0.9600 & $\mathrm{C}(25)-\mathrm{H}(25)$ & 0.9300 \\
\hline $\mathrm{C}(13)-\mathrm{H}(13 \mathrm{C})$ & 0.9600 & $C(26)-C(27)$ & $1.381(7)$ \\
\hline $\mathrm{C}(14)-\mathrm{H}(14 \mathrm{~A})$ & 0.9600 & $\mathrm{C}(26)-\mathrm{H}(26)$ & 0.9300 \\
\hline $\mathrm{C}(14)-\mathrm{H}(14 \mathrm{~B})$ & 0.9600 & $\mathrm{C}(27)-\mathrm{C}(28)$ & $1.382(7)$ \\
\hline $\mathrm{C}(14)-\mathrm{H}(14 \mathrm{C})$ & 0.9600 & $\mathrm{C}(27)-\mathrm{H}(27)$ & 0.9300 \\
\hline$C(15)-C(16)$ & $1.513(7)$ & $\mathrm{C}(28)-\mathrm{C}(29)$ & $1.517(7)$ \\
\hline$C(15)-C(17)$ & $1.527(8)$ & $\mathrm{C}(29)-\mathrm{C}(31)$ & $1.515(7)$ \\
\hline $\mathrm{C}(15)-\mathrm{H}(15)$ & 0.9800 & $C(29)-C(30)$ & $1.519(7)$ \\
\hline $\mathrm{C}(16)-\mathrm{H}(16 \mathrm{~A})$ & 0.9600 & $\mathrm{C}(29)-\mathrm{H}(29)$ & 0.9800 \\
\hline $\mathrm{C}(16)-\mathrm{H}(16 \mathrm{~B})$ & 0.9600 & $\mathrm{C}(30)-\mathrm{H}(30 \mathrm{~A})$ & 0.9600 \\
\hline$C(16)-H(16 C)$ & 0.9600 & $\mathrm{C}(30)-\mathrm{H}(30 \mathrm{~B})$ & 0.9600 \\
\hline $\mathrm{C}(17)-\mathrm{H}(17 \mathrm{~A})$ & 0.9600 & $\mathrm{C}(30)-\mathrm{H}(30 \mathrm{C})$ & 0.9600 \\
\hline $\mathrm{C}(17)-\mathrm{H}(17 \mathrm{~B})$ & 0.9600 & $\mathrm{C}(31)-\mathrm{H}(31 \mathrm{~A})$ & 0.9600 \\
\hline $\mathrm{C}(17)-\mathrm{H}(17 \mathrm{C})$ & 0.9600 & $\mathrm{C}(31)-\mathrm{H}(31 \mathrm{~B})$ & 0.9600 \\
\hline
\end{tabular}




\begin{tabular}{|c|c|c|c|}
\hline $\mathrm{C}(31)-\mathrm{H}(31 \mathrm{C})$ & 0.9600 & $\mathrm{C}(41)-\mathrm{H}(41)$ & 0.9300 \\
\hline$C(32)-C(34)$ & $1.515(8)$ & $\mathrm{C}(42)-\mathrm{H}(42 \mathrm{~A})$ & 0.9700 \\
\hline$C(32)-C(33)$ & $1.537(7)$ & $\mathrm{C}(42)-\mathrm{H}(42 \mathrm{~B})$ & 0.9700 \\
\hline $\mathrm{C}(32)-\mathrm{H}(32)$ & 0.9800 & $\mathrm{C}(43)-\mathrm{C}(44)$ & $1.377(7)$ \\
\hline $\mathrm{C}(33)-\mathrm{H}(33 \mathrm{~A})$ & 0.9600 & $\mathrm{C}(43)-\mathrm{H}(43)$ & 0.9300 \\
\hline $\mathrm{C}(33)-\mathrm{H}(33 \mathrm{~B})$ & 0.9600 & $C(44)-C(45)$ & $1.385(7)$ \\
\hline $\mathrm{C}(33)-\mathrm{H}(33 \mathrm{C})$ & 0.9600 & $\mathrm{C}(44)-\mathrm{H}(44)$ & 0.9300 \\
\hline $\mathrm{C}(34)-\mathrm{H}(34 \mathrm{~A})$ & 0.9600 & $C(45)-C(46)$ & $1.393(7)$ \\
\hline $\mathrm{C}(34)-\mathrm{H}(34 \mathrm{~B})$ & 0.9600 & $\mathrm{C}(45)-\mathrm{H}(45)$ & 0.9300 \\
\hline $\mathrm{C}(34)-\mathrm{H}(34 \mathrm{C})$ & 0.9600 & $\mathrm{C}(46)-\mathrm{C}(47)$ & $1.413(7)$ \\
\hline $\mathrm{C}(35)-\mathrm{H}(35 \mathrm{~A})$ & 0.9700 & $\mathrm{C}(47)-\mathrm{H}(47)$ & 0.9300 \\
\hline $\mathrm{C}(35)-\mathrm{H}(35 \mathrm{~B})$ & 0.9700 & $C(48)-C(49)$ & $1.399(7)$ \\
\hline$C(36)-H(36 A)$ & 0.9600 & $\mathrm{C}(48)-\mathrm{C}(53)$ & $1.400(7)$ \\
\hline $\mathrm{C}(36)-\mathrm{H}(36 \mathrm{~B})$ & 0.9600 & $\mathrm{C}(49)-\mathrm{C}(50)$ & $1.396(7)$ \\
\hline $\mathrm{C}(36)-\mathrm{H}(36 \mathrm{C})$ & 0.9600 & $C(49)-C(57)$ & $1.516(7)$ \\
\hline $\mathrm{C}(37)-\mathrm{H}(37 \mathrm{~A})$ & 0.9600 & $\mathrm{C}(50)-\mathrm{C}(51)$ & $1.363(7)$ \\
\hline $\mathrm{C}(37)-\mathrm{H}(37 \mathrm{~B})$ & 0.9600 & $\mathrm{C}(50)-\mathrm{H}(50)$ & 0.9300 \\
\hline $\mathrm{C}(37)-\mathrm{H}(37 \mathrm{C})$ & 0.9600 & $\mathrm{C}(51)-\mathrm{C}(52)$ & $1.365(8)$ \\
\hline $\mathrm{C}(38)-\mathrm{H}(38 \mathrm{~A})$ & 0.9600 & $\mathrm{C}(51)-\mathrm{H}(51)$ & 0.9300 \\
\hline $\mathrm{C}(38)-\mathrm{H}(38 \mathrm{~B})$ & 0.9600 & $\mathrm{C}(52)-\mathrm{C}(53)$ & $1.398(7)$ \\
\hline $\mathrm{C}(38)-\mathrm{H}(38 \mathrm{C})$ & 0.9600 & $\mathrm{C}(52)-\mathrm{H}(52)$ & 0.9300 \\
\hline $\mathrm{C}(39)-\mathrm{C}(40)$ & $1.473(8)$ & $\mathrm{C}(53)-\mathrm{C}(54)$ & $1.512(8)$ \\
\hline $\mathrm{C}(39)-\mathrm{H}(39 \mathrm{~A})$ & 0.9700 & $\mathrm{C}(54)-\mathrm{C}(56)$ & $1.510(9)$ \\
\hline $\mathrm{C}(39)-\mathrm{H}(39 \mathrm{~B})$ & 0.9700 & $C(54)-C(55)$ & $1.536(9)$ \\
\hline $\mathrm{C}(40)-\mathrm{C}(41)$ & $1.330(10)$ & $\mathrm{C}(54)-\mathrm{H}(54)$ & 0.9800 \\
\hline $\mathrm{C}(40)-\mathrm{H}(40)$ & 0.9300 & $\mathrm{C}(55)-\mathrm{H}(55 \mathrm{~A})$ & 0.9600 \\
\hline$C(41)-C(42)$ & $1.482(8)$ & $\mathrm{C}(55)-\mathrm{H}(55 \mathrm{~B})$ & 0.9600 \\
\hline
\end{tabular}




\begin{tabular}{|c|c|c|c|}
\hline $\mathrm{C}(55)-\mathrm{H}(55 \mathrm{C})$ & 0.9600 & $C(68)-C(69)$ & $1.382(8)$ \\
\hline $\mathrm{C}(56)-\mathrm{H}(56 \mathrm{~A})$ & 0.9600 & $\mathrm{C}(68)-\mathrm{H}(68)$ & 0.9300 \\
\hline $\mathrm{C}(56)-\mathrm{H}(56 \mathrm{~B})$ & 0.9600 & $C(69)-C(70)$ & $1.378(7)$ \\
\hline $\mathrm{C}(56)-\mathrm{H}(56 \mathrm{C})$ & 0.9600 & $\mathrm{C}(69)-\mathrm{H}(69)$ & 0.9300 \\
\hline $\mathrm{C}(57)-\mathrm{C}(58)$ & $1.519(7)$ & $\mathrm{C}(70)-\mathrm{C}(71)$ & $1.525(7)$ \\
\hline $\mathrm{C}(57)-\mathrm{C}(59)$ & $1.525(8)$ & $\mathrm{C}(71)-\mathrm{C}(73)$ & $1.506(8)$ \\
\hline $\mathrm{C}(57)-\mathrm{H}(57)$ & 0.9800 & $\mathrm{C}(71)-\mathrm{C}(72)$ & $1.520(8)$ \\
\hline $\mathrm{C}(58)-\mathrm{H}(58 \mathrm{~A})$ & 0.9600 & $\mathrm{C}(71)-\mathrm{H}(71)$ & 0.9800 \\
\hline $\mathrm{C}(58)-\mathrm{H}(58 \mathrm{~B})$ & 0.9600 & $\mathrm{C}(72)-\mathrm{H}(72 \mathrm{~A})$ & 0.9600 \\
\hline $\mathrm{C}(58)-\mathrm{H}(58 \mathrm{C})$ & 0.9600 & $\mathrm{C}(72)-\mathrm{H}(72 \mathrm{~B})$ & 0.9600 \\
\hline $\mathrm{C}(59)-\mathrm{H}(59 \mathrm{~A})$ & 0.9600 & $\mathrm{C}(72)-\mathrm{H}(72 \mathrm{C})$ & 0.9600 \\
\hline $\mathrm{C}(59)-\mathrm{H}(59 \mathrm{~B})$ & 0.9600 & $\mathrm{C}(73)-\mathrm{H}(73 \mathrm{~A})$ & 0.9600 \\
\hline $\mathrm{C}(59)-\mathrm{H}(59 \mathrm{C})$ & 0.9600 & $\mathrm{C}(73)-\mathrm{H}(73 \mathrm{~B})$ & 0.9600 \\
\hline $\mathrm{C}(60)-\mathrm{C}(61)$ & $1.379(8)$ & $\mathrm{C}(73)-\mathrm{H}(73 \mathrm{C})$ & 0.9600 \\
\hline $\mathrm{C}(60)-\mathrm{H}(60)$ & 0.9300 & $C(74)-C(76)$ & $1.509(8)$ \\
\hline$C(61)-C(62)$ & $1.373(8)$ & $C(74)-C(75)$ & $1.524(8)$ \\
\hline $\mathrm{C}(61)-\mathrm{H}(61)$ & 0.9300 & $\mathrm{C}(74)-\mathrm{H}(74)$ & 0.9800 \\
\hline$C(62)-C(63)$ & $1.410(7)$ & $\mathrm{C}(75)-\mathrm{H}(75 \mathrm{~A})$ & 0.9600 \\
\hline $\mathrm{C}(62)-\mathrm{H}(62)$ & 0.9300 & $\mathrm{C}(75)-\mathrm{H}(75 \mathrm{~B})$ & 0.9600 \\
\hline$C(63)-C(64)$ & $1.402(7)$ & $\mathrm{C}(75)-\mathrm{H}(75 \mathrm{C})$ & 0.9600 \\
\hline $\mathrm{C}(64)-\mathrm{H}(64)$ & 0.9300 & $\mathrm{C}(76)-\mathrm{H}(76 \mathrm{~A})$ & 0.9600 \\
\hline$C(65)-C(66)$ & $1.396(7)$ & $\mathrm{C}(76)-\mathrm{H}(76 \mathrm{~B})$ & 0.9600 \\
\hline $\mathrm{C}(65)-\mathrm{C}(70)$ & $1.414(7)$ & $\mathrm{C}(76)-\mathrm{H}(76 \mathrm{C})$ & 0.9600 \\
\hline$C(66)-C(67)$ & $1.393(7)$ & $\mathrm{C}(77)-\mathrm{H}(77 \mathrm{~A})$ & 0.9700 \\
\hline$C(66)-C(74)$ & $1.519(7)$ & $\mathrm{C}(77)-\mathrm{H}(77 \mathrm{~B})$ & 0.9700 \\
\hline$C(67)-C(68)$ & $1.377(7)$ & $\mathrm{C}(78)-\mathrm{H}(78 \mathrm{~A})$ & 0.9600 \\
\hline $\mathrm{C}(67)-\mathrm{H}(67)$ & 0.9300 & $\mathrm{C}(78)-\mathrm{H}(78 \mathrm{~B})$ & 0.9600 \\
\hline
\end{tabular}




\begin{tabular}{|c|c|c|c|}
\hline $\mathrm{C}(78)-\mathrm{H}(78 \mathrm{C})$ & 0.9600 & $\mathrm{C}(81)-\mathrm{H}(81 \mathrm{~A})$ & 0.9700 \\
\hline $\mathrm{C}(79)-\mathrm{H}(79 \mathrm{~A})$ & 0.9600 & $\mathrm{C}(81)-\mathrm{H}(81 \mathrm{~B})$ & 0.9700 \\
\hline $\mathrm{C}(79)-\mathrm{H}(79 \mathrm{~B})$ & 0.9600 & $C(82)-C(83)$ & $1.326(9)$ \\
\hline $\mathrm{C}(79)-\mathrm{H}(79 \mathrm{C})$ & 0.9600 & $\mathrm{C}(82)-\mathrm{H}(82)$ & 0.9300 \\
\hline $\mathrm{C}(80)-\mathrm{H}(80 \mathrm{~A})$ & 0.9600 & $\mathrm{C}(83)-\mathrm{C}(84)$ & $1.502(9)$ \\
\hline $\mathrm{C}(80)-\mathrm{H}(80 \mathrm{~B})$ & 0.9600 & $\mathrm{C}(83)-\mathrm{H}(83)$ & 0.9300 \\
\hline $\mathrm{C}(80)-\mathrm{H}(80 \mathrm{C})$ & 0.9600 & $\mathrm{C}(84)-\mathrm{H}(84 \mathrm{~A})$ & 0.9700 \\
\hline $\mathrm{C}(81)-\mathrm{C}(82)$ & $1.475(8)$ & $\mathrm{C}(84)-\mathrm{H}(84 \mathrm{~B})$ & 0.9700 \\
\hline $\mathrm{N}(1)-\mathrm{Lu}(1)-\mathrm{O}(1)$ & $83.29(13)$ & $\mathrm{O}(2)-\mathrm{Lu}(2)-\mathrm{C}(77)$ & $95.02(16)$ \\
\hline $\mathrm{N}(1)-\mathrm{Lu}(1)-\mathrm{N}(3)$ & $86.56(15)$ & $\mathrm{N}(7)-\mathrm{Lu}(2)-\mathrm{C}(77)$ & $165.38(17)$ \\
\hline $\mathrm{O}(1)-\mathrm{Lu}(1)-\mathrm{N}(3)$ & $84.02(13)$ & $\mathrm{N}(5)-\mathrm{Lu}(2)-\mathrm{N}(8)$ & $156.29(15)$ \\
\hline $\mathrm{N}(1)-\mathrm{Lu}(1)-\mathrm{C}(35)$ & $108.69(17)$ & $\mathrm{O}(2)-\mathrm{Lu}(2)-\mathrm{N}(8)$ & $90.11(13)$ \\
\hline $\mathrm{O}(1)-\mathrm{Lu}(1)-\mathrm{C}(35)$ & $95.49(16)$ & $\mathrm{N}(7)-\mathrm{Lu}(2)-\mathrm{N}(8)$ & $70.53(15)$ \\
\hline $\mathrm{N}(3)-\mathrm{Lu}(1)-\mathrm{C}(35)$ & $164.61(17)$ & $\mathrm{C}(77)-\mathrm{Lu}(2)-\mathrm{N}(8)$ & $94.91(17)$ \\
\hline $\mathrm{N}(1)-\mathrm{Lu}(1)-\mathrm{N}(4)$ & $156.99(14)$ & $\mathrm{N}(5)-\mathrm{Lu}(2)-\mathrm{N}(6)$ & $73.85(14)$ \\
\hline $\mathrm{O}(1)-\mathrm{Lu}(1)-\mathrm{N}(4)$ & $90.71(13)$ & $\mathrm{O}(2)-\mathrm{Lu}(2)-\mathrm{N}(6)$ & $156.18(13)$ \\
\hline $\mathrm{N}(3)-\mathrm{Lu}(1)-\mathrm{N}(4)$ & $70.69(14)$ & $\mathrm{N}(7)-\mathrm{Lu}(2)-\mathrm{N}(6)$ & $87.26(14)$ \\
\hline $\mathrm{C}(35)-\mathrm{Lu}(1)-\mathrm{N}(4)$ & $93.95(16)$ & $C(77)-\mathrm{Lu}(2)-\mathrm{N}(6)$ & $98.84(17)$ \\
\hline $\mathrm{N}(1)-\mathrm{Lu}(1)-\mathrm{N}(2)$ & $73.39(14)$ & $\mathrm{N}(8)-\mathrm{Lu}(2)-\mathrm{N}(6)$ & $107.79(13)$ \\
\hline $\mathrm{O}(1)-\mathrm{Lu}(1)-\mathrm{N}(2)$ & $155.72(13)$ & $\mathrm{C}(38)-\mathrm{Si}(1)-\mathrm{C}(36)$ & $105.3(3)$ \\
\hline $\mathrm{N}(3)-\mathrm{Lu}(1)-\mathrm{N}(2)$ & $87.88(14)$ & $\mathrm{C}(38)-\mathrm{Si}(1)-\mathrm{C}(37)$ & $103.9(3)$ \\
\hline $\mathrm{C}(35)-\mathrm{Lu}(1)-\mathrm{N}(2)$ & $98.25(17)$ & $C(36)-\mathrm{Si}(1)-\mathrm{C}(37)$ & $108.8(4)$ \\
\hline $\mathrm{N}(4)-\mathrm{Lu}(1)-\mathrm{N}(2)$ & $108.13(14)$ & $\mathrm{C}(38)-\mathrm{Si}(1)-\mathrm{C}(35)$ & $117.9(3)$ \\
\hline $\mathrm{N}(5)-\mathrm{Lu}(2)-\mathrm{O}(2)$ & $83.45(14)$ & $\mathrm{C}(36)-\mathrm{Si}(1)-\mathrm{C}(35)$ & $111.3(3)$ \\
\hline $\mathrm{N}(5)-\mathrm{Lu}(2)-\mathrm{N}(7)$ & $86.08(15)$ & $\mathrm{C}(37)-\mathrm{Si}(1)-\mathrm{C}(35)$ & $109.0(3)$ \\
\hline $\mathrm{O}(2)-\mathrm{Lu}(2)-\mathrm{N}(7)$ & $84.01(13)$ & $C(78)-\mathrm{Si}(5)-\mathrm{C}(80)$ & $104.6(3)$ \\
\hline $\mathrm{N}(5)-\mathrm{Lu}(2)-\mathrm{C}(77)$ & $108.34(17)$ & $\mathrm{C}(78)-\mathrm{Si}(5)-\mathrm{C}(79)$ & $107.8(4)$ \\
\hline
\end{tabular}




\begin{tabular}{|c|c|c|c|}
\hline $\mathrm{C}(80)-\mathrm{Si}(5)-\mathrm{C}(79)$ & $105.1(3)$ & $\mathrm{C}(48)-\mathrm{N}(6)-\mathrm{Lu}(2)$ & $135.1(3)$ \\
\hline $\mathrm{C}(78)-\mathrm{Si}(5)-\mathrm{C}(77)$ & $109.7(3)$ & $\mathrm{C}(60)-\mathrm{N}(7)-\mathrm{C}(63)$ & $106.2(5)$ \\
\hline $\mathrm{C}(80)-\mathrm{Si}(5)-\mathrm{C}(77)$ & $116.4(3)$ & $\mathrm{C}(60)-\mathrm{N}(7)-\mathrm{Lu}(2)$ & $137.6(4)$ \\
\hline $\mathrm{C}(79)-\mathrm{Si}(5)-\mathrm{C}(77)$ & $112.7(3)$ & $\mathrm{C}(63)-\mathrm{N}(7)-\mathrm{Lu}(2)$ & $115.4(3)$ \\
\hline $\mathrm{C}(42)-\mathrm{O}(1)-\mathrm{C}(39)$ & $108.1(4)$ & $C(64)-N(8)-C(65)$ & $116.3(5)$ \\
\hline $\mathrm{C}(42)-\mathrm{O}(1)-\mathrm{Lu}(1)$ & $124.7(3)$ & $\mathrm{C}(64)-\mathrm{N}(8)-\mathrm{Lu}(2)$ & $112.9(3)$ \\
\hline $\mathrm{C}(39)-\mathrm{O}(1)-\mathrm{Lu}(1)$ & $125.3(3)$ & $\mathrm{C}(65)-\mathrm{N}(8)-\mathrm{Lu}(2)$ & $130.4(3)$ \\
\hline $\mathrm{C}(81)-\mathrm{O}(2)-\mathrm{C}(84)$ & $108.0(4)$ & $\mathrm{N}(1)-\mathrm{C}(1)-\mathrm{C}(2)$ & $111.0(5)$ \\
\hline $\mathrm{C}(81)-\mathrm{O}(2)-\mathrm{Lu}(2)$ & $125.4(3)$ & $\mathrm{N}(1)-\mathrm{C}(1)-\mathrm{H}(1)$ & 124.5 \\
\hline $\mathrm{C}(84)-\mathrm{O}(2)-\mathrm{Lu}(2)$ & $124.5(3)$ & $\mathrm{C}(2)-\mathrm{C}(1)-\mathrm{H}(1)$ & 124.5 \\
\hline $\mathrm{C}(1)-\mathrm{N}(1)-\mathrm{C}(4)$ & $105.0(4)$ & $C(3)-C(2)-C(1)$ & $106.8(5)$ \\
\hline $\mathrm{C}(1)-\mathrm{N}(1)-\mathrm{Lu}(1)$ & $141.4(4)$ & $\mathrm{C}(3)-\mathrm{C}(2)-\mathrm{H}(2)$ & 126.6 \\
\hline $\mathrm{C}(4)-\mathrm{N}(1)-\mathrm{Lu}(1)$ & $113.5(3)$ & $\mathrm{C}(1)-\mathrm{C}(2)-\mathrm{H}(2)$ & 126.6 \\
\hline$C(5)-N(2)-C(6)$ & $116.6(4)$ & $C(2)-C(3)-C(4)$ & $106.5(5)$ \\
\hline $\mathrm{C}(5)-\mathrm{N}(2)-\mathrm{Lu}(1)$ & $109.8(3)$ & $\mathrm{C}(2)-\mathrm{C}(3)-\mathrm{H}(3)$ & 126.8 \\
\hline $\mathrm{C}(6)-\mathrm{N}(2)-\mathrm{Lu}(1)$ & $133.5(3)$ & $\mathrm{C}(4)-\mathrm{C}(3)-\mathrm{H}(3)$ & 126.8 \\
\hline $\mathrm{C}(18)-\mathrm{N}(3)-\mathrm{C}(21)$ & $104.6(4)$ & $\mathrm{N}(1)-\mathrm{C}(4)-\mathrm{C}(3)$ & $110.7(5)$ \\
\hline $\mathrm{C}(18)-\mathrm{N}(3)-\mathrm{Lu}(1)$ & $139.0(4)$ & $N(1)-C(4)-C(5)$ & $120.3(5)$ \\
\hline $\mathrm{C}(21)-\mathrm{N}(3)-\mathrm{Lu}(1)$ & $115.7(3)$ & $C(3)-C(4)-C(5)$ & $129.1(5)$ \\
\hline$C(22)-N(4)-C(23)$ & $115.8(4)$ & $N(2)-C(5)-C(4)$ & $123.0(5)$ \\
\hline $\mathrm{C}(22)-\mathrm{N}(4)-\mathrm{Lu}(1)$ & $113.4(3)$ & $\mathrm{N}(2)-\mathrm{C}(5)-\mathrm{H}(5)$ & 118.5 \\
\hline $\mathrm{C}(23)-\mathrm{N}(4)-\mathrm{Lu}(1)$ & $130.6(3)$ & $\mathrm{C}(4)-\mathrm{C}(5)-\mathrm{H}(5)$ & 118.5 \\
\hline $\mathrm{C}(43)-\mathrm{N}(5)-\mathrm{C}(46)$ & $105.2(4)$ & $C(7)-C(6)-C(11)$ & $121.7(5)$ \\
\hline $\mathrm{C}(43)-\mathrm{N}(5)-\mathrm{Lu}(2)$ & $141.4(4)$ & $C(7)-C(6)-N(2)$ & $119.3(5)$ \\
\hline $\mathrm{C}(46)-\mathrm{N}(5)-\mathrm{Lu}(2)$ & $113.2(3)$ & $\mathrm{C}(11)-\mathrm{C}(6)-\mathrm{N}(2)$ & $119.0(5)$ \\
\hline $\mathrm{C}(47)-\mathrm{N}(6)-\mathrm{C}(48)$ & $116.1(4)$ & $C(8)-C(7)-C(6)$ & $117.6(5)$ \\
\hline $\mathrm{C}(47)-\mathrm{N}(6)-\mathrm{Lu}(2)$ & $108.8(3)$ & $C(8)-C(7)-C(15)$ & $120.0(5)$ \\
\hline
\end{tabular}




\begin{tabular}{|c|c|c|c|}
\hline$C(6)-C(7)-C(15)$ & $122.3(5)$ & $\mathrm{H}(14 \mathrm{~A})-\mathrm{C}(14)-\mathrm{H}(14 \mathrm{~B})$ & 109.5 \\
\hline$C(9)-C(8)-C(7)$ & $121.5(5)$ & $\mathrm{C}(12)-\mathrm{C}(14)-\mathrm{H}(14 \mathrm{C})$ & 109.5 \\
\hline $\mathrm{C}(9)-\mathrm{C}(8)-\mathrm{H}(8)$ & 119.3 & $\mathrm{H}(14 \mathrm{~A})-\mathrm{C}(14)-\mathrm{H}(14 \mathrm{C})$ & 109.5 \\
\hline $\mathrm{C}(7)-\mathrm{C}(8)-\mathrm{H}(8)$ & 119.3 & $\mathrm{H}(14 \mathrm{~B})-\mathrm{C}(14)-\mathrm{H}(14 \mathrm{C})$ & 109.5 \\
\hline$C(10)-C(9)-C(8)$ & $119.9(5)$ & $C(7)-C(15)-C(16)$ & $114.2(5)$ \\
\hline $\mathrm{C}(10)-\mathrm{C}(9)-\mathrm{H}(9)$ & 120.0 & $C(7)-C(15)-C(17)$ & $110.9(5)$ \\
\hline $\mathrm{C}(8)-\mathrm{C}(9)-\mathrm{H}(9)$ & 120.0 & $C(16)-C(15)-C(17)$ & $107.9(5)$ \\
\hline$C(9)-C(10)-C(11)$ & $121.1(5)$ & $\mathrm{C}(7)-\mathrm{C}(15)-\mathrm{H}(15)$ & 107.9 \\
\hline $\mathrm{C}(9)-\mathrm{C}(10)-\mathrm{H}(10)$ & 119.4 & $\mathrm{C}(16)-\mathrm{C}(15)-\mathrm{H}(15)$ & 107.9 \\
\hline $\mathrm{C}(11)-\mathrm{C}(10)-\mathrm{H}(10)$ & 119.4 & $\mathrm{C}(17)-\mathrm{C}(15)-\mathrm{H}(15)$ & 107.9 \\
\hline$C(10)-C(11)-C(6)$ & $118.0(5)$ & $\mathrm{C}(15)-\mathrm{C}(16)-\mathrm{H}(16 \mathrm{~A})$ & 109.5 \\
\hline$C(10)-C(11)-C(12)$ & $119.2(5)$ & $\mathrm{C}(15)-\mathrm{C}(16)-\mathrm{H}(16 \mathrm{~B})$ & 109.5 \\
\hline$C(6)-C(11)-C(12)$ & $122.8(5)$ & $\mathrm{H}(16 \mathrm{~A})-\mathrm{C}(16)-\mathrm{H}(16 \mathrm{~B})$ & 109.5 \\
\hline $\mathrm{C}(14)-\mathrm{C}(12)-\mathrm{C}(11)$ & $115.4(5)$ & $\mathrm{C}(15)-\mathrm{C}(16)-\mathrm{H}(16 \mathrm{C})$ & 109.5 \\
\hline$C(14)-C(12)-C(13)$ & $110.3(5)$ & $\mathrm{H}(16 \mathrm{~A})-\mathrm{C}(16)-\mathrm{H}(16 \mathrm{C})$ & 109.5 \\
\hline$C(11)-C(12)-C(13)$ & $107.9(5)$ & $\mathrm{H}(16 \mathrm{~B})-\mathrm{C}(16)-\mathrm{H}(16 \mathrm{C})$ & 109.5 \\
\hline $\mathrm{C}(14)-\mathrm{C}(12)-\mathrm{H}(12)$ & 107.6 & $\mathrm{C}(15)-\mathrm{C}(17)-\mathrm{H}(17 \mathrm{~A})$ & 109.5 \\
\hline $\mathrm{C}(11)-\mathrm{C}(12)-\mathrm{H}(12)$ & 107.6 & $\mathrm{C}(15)-\mathrm{C}(17)-\mathrm{H}(17 \mathrm{~B})$ & 109.5 \\
\hline $\mathrm{C}(13)-\mathrm{C}(12)-\mathrm{H}(12)$ & 107.6 & $\mathrm{H}(17 \mathrm{~A})-\mathrm{C}(17)-\mathrm{H}(17 \mathrm{~B})$ & 109.5 \\
\hline $\mathrm{C}(12)-\mathrm{C}(13)-\mathrm{H}(13 \mathrm{~A})$ & 109.5 & $\mathrm{C}(15)-\mathrm{C}(17)-\mathrm{H}(17 \mathrm{C})$ & 109.5 \\
\hline $\mathrm{C}(12)-\mathrm{C}(13)-\mathrm{H}(13 \mathrm{~B})$ & 109.5 & $\mathrm{H}(17 \mathrm{~A})-\mathrm{C}(17)-\mathrm{H}(17 \mathrm{C})$ & 109.5 \\
\hline $\mathrm{H}(13 \mathrm{~A})-\mathrm{C}(13)-\mathrm{H}(13 \mathrm{~B})$ & 109.5 & $\mathrm{H}(17 \mathrm{~B})-\mathrm{C}(17)-\mathrm{H}(17 \mathrm{C})$ & 109.5 \\
\hline $\mathrm{C}(12)-\mathrm{C}(13)-\mathrm{H}(13 \mathrm{C})$ & 109.5 & $\mathrm{~N}(3)-\mathrm{C}(18)-\mathrm{C}(19)$ & $111.9(5)$ \\
\hline $\mathrm{H}(13 \mathrm{~A})-\mathrm{C}(13)-\mathrm{H}(13 \mathrm{C})$ & 109.5 & $\mathrm{~N}(3)-\mathrm{C}(18)-\mathrm{H}(18)$ & 124.1 \\
\hline $\mathrm{H}(13 \mathrm{~B})-\mathrm{C}(13)-\mathrm{H}(13 \mathrm{C})$ & 109.5 & $\mathrm{C}(19)-\mathrm{C}(18)-\mathrm{H}(18)$ & 124.1 \\
\hline $\mathrm{C}(12)-\mathrm{C}(14)-\mathrm{H}(14 \mathrm{~A})$ & 109.5 & $C(20)-C(19)-C(18)$ & $106.9(5)$ \\
\hline $\mathrm{C}(12)-\mathrm{C}(14)-\mathrm{H}(14 \mathrm{~B})$ & 109.5 & $\mathrm{C}(20)-\mathrm{C}(19)-\mathrm{H}(19)$ & 126.5 \\
\hline
\end{tabular}




\begin{tabular}{|c|c|c|c|}
\hline $\mathrm{C}(18)-\mathrm{C}(19)-\mathrm{H}(19)$ & 126.5 & $\mathrm{C}(23)-\mathrm{C}(28)-\mathrm{C}(29)$ & $121.4(5)$ \\
\hline$C(19)-C(20)-C(21)$ & $106.0(5)$ & $\mathrm{C}(31)-\mathrm{C}(29)-\mathrm{C}(28)$ & $113.0(5)$ \\
\hline $\mathrm{C}(19)-\mathrm{C}(20)-\mathrm{H}(20)$ & 127.0 & $\mathrm{C}(31)-\mathrm{C}(29)-\mathrm{C}(30)$ & $110.3(5)$ \\
\hline $\mathrm{C}(21)-\mathrm{C}(20)-\mathrm{H}(20)$ & 127.0 & $C(28)-C(29)-C(30)$ & $111.0(5)$ \\
\hline $\mathrm{N}(3)-\mathrm{C}(21)-\mathrm{C}(20)$ & $110.5(5)$ & $\mathrm{C}(31)-\mathrm{C}(29)-\mathrm{H}(29)$ & 107.4 \\
\hline $\mathrm{N}(3)-\mathrm{C}(21)-\mathrm{C}(22)$ & $118.3(5)$ & $\mathrm{C}(28)-\mathrm{C}(29)-\mathrm{H}(29)$ & 107.4 \\
\hline$C(20)-C(21)-C(22)$ & $131.1(6)$ & $\mathrm{C}(30)-\mathrm{C}(29)-\mathrm{H}(29)$ & 107.4 \\
\hline $\mathrm{N}(4)-\mathrm{C}(22)-\mathrm{C}(21)$ & $121.7(5)$ & $\mathrm{C}(29)-\mathrm{C}(30)-\mathrm{H}(30 \mathrm{~A})$ & 109.5 \\
\hline $\mathrm{N}(4)-\mathrm{C}(22)-\mathrm{H}(22)$ & 119.1 & $\mathrm{C}(29)-\mathrm{C}(30)-\mathrm{H}(30 \mathrm{~B})$ & 109.5 \\
\hline $\mathrm{C}(21)-\mathrm{C}(22)-\mathrm{H}(22)$ & 119.1 & $\mathrm{H}(30 \mathrm{~A})-\mathrm{C}(30)-\mathrm{H}(30 \mathrm{~B})$ & 109.5 \\
\hline$C(28)-C(23)-C(24)$ & $121.4(5)$ & $\mathrm{C}(29)-\mathrm{C}(30)-\mathrm{H}(30 \mathrm{C})$ & 109.5 \\
\hline $\mathrm{C}(28)-\mathrm{C}(23)-\mathrm{N}(4)$ & $119.6(5)$ & $\mathrm{H}(30 \mathrm{~A})-\mathrm{C}(30)-\mathrm{H}(30 \mathrm{C})$ & 109.5 \\
\hline $\mathrm{C}(24)-\mathrm{C}(23)-\mathrm{N}(4)$ & $119.0(4)$ & $\mathrm{H}(30 \mathrm{~B})-\mathrm{C}(30)-\mathrm{H}(30 \mathrm{C})$ & 109.5 \\
\hline$C(25)-C(24)-C(23)$ & $118.0(5)$ & $\mathrm{C}(29)-\mathrm{C}(31)-\mathrm{H}(31 \mathrm{~A})$ & 109.5 \\
\hline$C(25)-C(24)-C(32)$ & $120.2(5)$ & $\mathrm{C}(29)-\mathrm{C}(31)-\mathrm{H}(31 \mathrm{~B})$ & 109.5 \\
\hline$C(23)-C(24)-C(32)$ & $121.9(5)$ & $\mathrm{H}(31 \mathrm{~A})-\mathrm{C}(31)-\mathrm{H}(31 \mathrm{~B})$ & 109.5 \\
\hline$C(26)-C(25)-C(24)$ & $120.9(5)$ & $\mathrm{C}(29)-\mathrm{C}(31)-\mathrm{H}(31 \mathrm{C})$ & 109.5 \\
\hline $\mathrm{C}(26)-\mathrm{C}(25)-\mathrm{H}(25)$ & 119.5 & $\mathrm{H}(31 \mathrm{~A})-\mathrm{C}(31)-\mathrm{H}(31 \mathrm{C})$ & 109.5 \\
\hline $\mathrm{C}(24)-\mathrm{C}(25)-\mathrm{H}(25)$ & 119.5 & $\mathrm{H}(31 \mathrm{~B})-\mathrm{C}(31)-\mathrm{H}(31 \mathrm{C})$ & 109.5 \\
\hline$C(25)-C(26)-C(27)$ & $120.1(5)$ & $\mathrm{C}(24)-\mathrm{C}(32)-\mathrm{C}(34)$ & $112.6(5)$ \\
\hline $\mathrm{C}(25)-\mathrm{C}(26)-\mathrm{H}(26)$ & 120.0 & $C(24)-C(32)-C(33)$ & $112.0(4)$ \\
\hline $\mathrm{C}(27)-\mathrm{C}(26)-\mathrm{H}(26)$ & 120.0 & $C(34)-C(32)-C(33)$ & $110.0(5)$ \\
\hline$C(26)-C(27)-C(28)$ & $121.4(5)$ & $\mathrm{C}(24)-\mathrm{C}(32)-\mathrm{H}(32)$ & 107.3 \\
\hline $\mathrm{C}(26)-\mathrm{C}(27)-\mathrm{H}(27)$ & 119.3 & $\mathrm{C}(34)-\mathrm{C}(32)-\mathrm{H}(32)$ & 107.3 \\
\hline $\mathrm{C}(28)-\mathrm{C}(27)-\mathrm{H}(27)$ & 119.3 & $\mathrm{C}(33)-\mathrm{C}(32)-\mathrm{H}(32)$ & 107.3 \\
\hline $\mathrm{C}(27)-\mathrm{C}(28)-\mathrm{C}(23)$ & $118.0(5)$ & $\mathrm{C}(32)-\mathrm{C}(33)-\mathrm{H}(33 \mathrm{~A})$ & 109.5 \\
\hline$C(27)-C(28)-C(29)$ & $120.5(5)$ & $\mathrm{C}(32)-\mathrm{C}(33)-\mathrm{H}(33 \mathrm{~B})$ & 109.5 \\
\hline
\end{tabular}




\begin{tabular}{|c|c|c|c|}
\hline $\mathrm{H}(33 \mathrm{~A})-\mathrm{C}(33)-\mathrm{H}(33 \mathrm{~B})$ & 109.5 & $\mathrm{H}(37 \mathrm{~B})-\mathrm{C}(37)-\mathrm{H}(37 \mathrm{C})$ & 109.5 \\
\hline $\mathrm{C}(32)-\mathrm{C}(33)-\mathrm{H}(33 \mathrm{C})$ & 109.5 & $\mathrm{Si}(1)-\mathrm{C}(38)-\mathrm{H}(38 \mathrm{~A})$ & 109.5 \\
\hline $\mathrm{H}(33 \mathrm{~A})-\mathrm{C}(33)-\mathrm{H}(33 \mathrm{C})$ & 109.5 & $\mathrm{Si}(1)-\mathrm{C}(38)-\mathrm{H}(38 \mathrm{~B})$ & 109.5 \\
\hline $\mathrm{H}(33 \mathrm{~B})-\mathrm{C}(33)-\mathrm{H}(33 \mathrm{C})$ & 109.5 & $\mathrm{H}(38 \mathrm{~A})-\mathrm{C}(38)-\mathrm{H}(38 \mathrm{~B})$ & 109.5 \\
\hline $\mathrm{C}(32)-\mathrm{C}(34)-\mathrm{H}(34 \mathrm{~A})$ & 109.5 & $\mathrm{Si}(1)-\mathrm{C}(38)-\mathrm{H}(38 \mathrm{C})$ & 109.5 \\
\hline $\mathrm{C}(32)-\mathrm{C}(34)-\mathrm{H}(34 \mathrm{~B})$ & 109.5 & $\mathrm{H}(38 \mathrm{~A})-\mathrm{C}(38)-\mathrm{H}(38 \mathrm{C})$ & 109.5 \\
\hline $\mathrm{H}(34 \mathrm{~A})-\mathrm{C}(34)-\mathrm{H}(34 \mathrm{~B})$ & 109.5 & $\mathrm{H}(38 \mathrm{~B})-\mathrm{C}(38)-\mathrm{H}(38 \mathrm{C})$ & 109.5 \\
\hline $\mathrm{C}(32)-\mathrm{C}(34)-\mathrm{H}(34 \mathrm{C})$ & 109.5 & $\mathrm{O}(1)-\mathrm{C}(39)-\mathrm{C}(40)$ & $105.7(5)$ \\
\hline $\mathrm{H}(34 \mathrm{~A})-\mathrm{C}(34)-\mathrm{H}(34 \mathrm{C})$ & 109.5 & $\mathrm{O}(1)-\mathrm{C}(39)-\mathrm{H}(39 \mathrm{~A})$ & 110.6 \\
\hline $\mathrm{H}(34 \mathrm{~B})-\mathrm{C}(34)-\mathrm{H}(34 \mathrm{C})$ & 109.5 & $\mathrm{C}(40)-\mathrm{C}(39)-\mathrm{H}(39 \mathrm{~A})$ & 110.6 \\
\hline $\mathrm{Si}(1)-\mathrm{C}(35)-\mathrm{Lu}(1)$ & $130.0(3)$ & $\mathrm{O}(1)-\mathrm{C}(39)-\mathrm{H}(39 \mathrm{~B})$ & 110.6 \\
\hline $\mathrm{Si}(1)-\mathrm{C}(35)-\mathrm{H}(35 \mathrm{~A})$ & 104.8 & $\mathrm{C}(40)-\mathrm{C}(39)-\mathrm{H}(39 \mathrm{~B})$ & 110.6 \\
\hline $\mathrm{Lu}(1)-\mathrm{C}(35)-\mathrm{H}(35 \mathrm{~A})$ & 104.8 & $\mathrm{H}(39 \mathrm{~A})-\mathrm{C}(39)-\mathrm{H}(39 \mathrm{~B})$ & 108.7 \\
\hline $\mathrm{Si}(1)-\mathrm{C}(35)-\mathrm{H}(35 \mathrm{~B})$ & 104.8 & $\mathrm{C}(41)-\mathrm{C}(40)-\mathrm{C}(39)$ & $109.9(6)$ \\
\hline $\mathrm{Lu}(1)-\mathrm{C}(35)-\mathrm{H}(35 \mathrm{~B})$ & 104.8 & $\mathrm{C}(41)-\mathrm{C}(40)-\mathrm{H}(40)$ & 125.0 \\
\hline $\mathrm{H}(35 \mathrm{~A})-\mathrm{C}(35)-\mathrm{H}(35 \mathrm{~B})$ & 105.8 & $\mathrm{C}(39)-\mathrm{C}(40)-\mathrm{H}(40)$ & 125.0 \\
\hline $\mathrm{Si}(1)-\mathrm{C}(36)-\mathrm{H}(36 \mathrm{~A})$ & 109.5 & $\mathrm{C}(40)-\mathrm{C}(41)-\mathrm{C}(42)$ & $110.3(6)$ \\
\hline $\mathrm{Si}(1)-\mathrm{C}(36)-\mathrm{H}(36 \mathrm{~B})$ & 109.5 & $\mathrm{C}(40)-\mathrm{C}(41)-\mathrm{H}(41)$ & 124.9 \\
\hline $\mathrm{H}(36 \mathrm{~A})-\mathrm{C}(36)-\mathrm{H}(36 \mathrm{~B})$ & 109.5 & $\mathrm{C}(42)-\mathrm{C}(41)-\mathrm{H}(41)$ & 124.9 \\
\hline $\mathrm{Si}(1)-\mathrm{C}(36)-\mathrm{H}(36 \mathrm{C})$ & 109.5 & $\mathrm{O}(1)-\mathrm{C}(42)-\mathrm{C}(41)$ & $105.7(5)$ \\
\hline $\mathrm{H}(36 \mathrm{~A})-\mathrm{C}(36)-\mathrm{H}(36 \mathrm{C})$ & 109.5 & $\mathrm{O}(1)-\mathrm{C}(42)-\mathrm{H}(42 \mathrm{~A})$ & 110.6 \\
\hline $\mathrm{H}(36 \mathrm{~B})-\mathrm{C}(36)-\mathrm{H}(36 \mathrm{C})$ & 109.5 & $\mathrm{C}(41)-\mathrm{C}(42)-\mathrm{H}(42 \mathrm{~A})$ & 110.6 \\
\hline $\mathrm{Si}(1)-\mathrm{C}(37)-\mathrm{H}(37 \mathrm{~A})$ & 109.5 & $\mathrm{O}(1)-\mathrm{C}(42)-\mathrm{H}(42 \mathrm{~B})$ & 110.6 \\
\hline $\mathrm{Si}(1)-\mathrm{C}(37)-\mathrm{H}(37 \mathrm{~B})$ & 109.5 & $\mathrm{C}(41)-\mathrm{C}(42)-\mathrm{H}(42 \mathrm{~B})$ & 110.6 \\
\hline $\mathrm{H}(37 \mathrm{~A})-\mathrm{C}(37)-\mathrm{H}(37 \mathrm{~B})$ & 109.5 & $\mathrm{H}(42 \mathrm{~A})-\mathrm{C}(42)-\mathrm{H}(42 \mathrm{~B})$ & 108.7 \\
\hline $\mathrm{Si}(1)-\mathrm{C}(37)-\mathrm{H}(37 \mathrm{C})$ & 109.5 & $N(5)-C(43)-C(44)$ & $111.1(5)$ \\
\hline $\mathrm{H}(37 \mathrm{~A})-\mathrm{C}(37)-\mathrm{H}(37 \mathrm{C})$ & 109.5 & $\mathrm{~N}(5)-\mathrm{C}(43)-\mathrm{H}(43)$ & 124.4 \\
\hline
\end{tabular}




\begin{tabular}{|c|c|c|c|}
\hline $\mathrm{C}(44)-\mathrm{C}(43)-\mathrm{H}(43)$ & 124.4 & $\mathrm{C}(53)-\mathrm{C}(52)-\mathrm{H}(52)$ & 118.9 \\
\hline$C(43)-C(44)-C(45)$ & $107.3(5)$ & $C(52)-C(53)-C(48)$ & $117.3(5)$ \\
\hline $\mathrm{C}(43)-\mathrm{C}(44)-\mathrm{H}(44)$ & 126.4 & $C(52)-C(53)-C(54)$ & $120.1(5)$ \\
\hline $\mathrm{C}(45)-\mathrm{C}(44)-\mathrm{H}(44)$ & 126.4 & $C(48)-C(53)-C(54)$ & $122.5(5)$ \\
\hline$C(44)-C(45)-C(46)$ & $105.8(5)$ & $C(56)-C(54)-C(53)$ & $110.7(5)$ \\
\hline $\mathrm{C}(44)-\mathrm{C}(45)-\mathrm{H}(45)$ & 127.1 & $C(56)-C(54)-C(55)$ & $110.2(6)$ \\
\hline $\mathrm{C}(46)-\mathrm{C}(45)-\mathrm{H}(45)$ & 127.1 & $C(53)-C(54)-C(55)$ & $114.1(5)$ \\
\hline$N(5)-C(46)-C(45)$ & $110.6(5)$ & $\mathrm{C}(56)-\mathrm{C}(54)-\mathrm{H}(54)$ & 107.2 \\
\hline $\mathrm{N}(5)-\mathrm{C}(46)-\mathrm{C}(47)$ & $119.8(5)$ & $\mathrm{C}(53)-\mathrm{C}(54)-\mathrm{H}(54)$ & 107.2 \\
\hline$C(45)-C(46)-C(47)$ & $129.6(5)$ & $\mathrm{C}(55)-\mathrm{C}(54)-\mathrm{H}(54)$ & 107.2 \\
\hline $\mathrm{N}(6)-\mathrm{C}(47)-\mathrm{C}(46)$ & $124.3(5)$ & $\mathrm{C}(54)-\mathrm{C}(55)-\mathrm{H}(55 \mathrm{~A})$ & 109.5 \\
\hline $\mathrm{N}(6)-\mathrm{C}(47)-\mathrm{H}(47)$ & 117.9 & $\mathrm{C}(54)-\mathrm{C}(55)-\mathrm{H}(55 \mathrm{~B})$ & 109.5 \\
\hline $\mathrm{C}(46)-\mathrm{C}(47)-\mathrm{H}(47)$ & 117.9 & $\mathrm{H}(55 \mathrm{~A})-\mathrm{C}(55)-\mathrm{H}(55 \mathrm{~B})$ & 109.5 \\
\hline$C(49)-C(48)-C(53)$ & $121.7(5)$ & $\mathrm{C}(54)-\mathrm{C}(55)-\mathrm{H}(55 \mathrm{C})$ & 109.5 \\
\hline$C(49)-C(48)-N(6)$ & $119.1(5)$ & $\mathrm{H}(55 \mathrm{~A})-\mathrm{C}(55)-\mathrm{H}(55 \mathrm{C})$ & 109.5 \\
\hline$C(53)-C(48)-N(6)$ & $119.3(5)$ & $\mathrm{H}(55 \mathrm{~B})-\mathrm{C}(55)-\mathrm{H}(55 \mathrm{C})$ & 109.5 \\
\hline$C(50)-C(49)-C(48)$ & $117.3(5)$ & $\mathrm{C}(54)-\mathrm{C}(56)-\mathrm{H}(56 \mathrm{~A})$ & 109.5 \\
\hline$C(50)-C(49)-C(57)$ & $119.4(5)$ & $\mathrm{C}(54)-\mathrm{C}(56)-\mathrm{H}(56 \mathrm{~B})$ & 109.5 \\
\hline$C(48)-C(49)-C(57)$ & $123.2(5)$ & $\mathrm{H}(56 \mathrm{~A})-\mathrm{C}(56)-\mathrm{H}(56 \mathrm{~B})$ & 109.5 \\
\hline$C(51)-C(50)-C(49)$ & $122.4(6)$ & $\mathrm{C}(54)-\mathrm{C}(56)-\mathrm{H}(56 \mathrm{C})$ & 109.5 \\
\hline $\mathrm{C}(51)-\mathrm{C}(50)-\mathrm{H}(50)$ & 118.8 & $\mathrm{H}(56 \mathrm{~A})-\mathrm{C}(56)-\mathrm{H}(56 \mathrm{C})$ & 109.5 \\
\hline $\mathrm{C}(49)-\mathrm{C}(50)-\mathrm{H}(50)$ & 118.8 & $\mathrm{H}(56 \mathrm{~B})-\mathrm{C}(56)-\mathrm{H}(56 \mathrm{C})$ & 109.5 \\
\hline$C(50)-C(51)-C(52)$ & $119.2(5)$ & $C(49)-C(57)-C(58)$ & $113.9(5)$ \\
\hline $\mathrm{C}(50)-\mathrm{C}(51)-\mathrm{H}(51)$ & 120.4 & $C(49)-C(57)-C(59)$ & $111.0(5)$ \\
\hline $\mathrm{C}(52)-\mathrm{C}(51)-\mathrm{H}(51)$ & 120.4 & $C(58)-C(57)-C(59)$ & $109.3(5)$ \\
\hline$C(51)-C(52)-C(53)$ & $122.1(6)$ & $\mathrm{C}(49)-\mathrm{C}(57)-\mathrm{H}(57)$ & 107.5 \\
\hline $\mathrm{C}(51)-\mathrm{C}(52)-\mathrm{H}(52)$ & 118.9 & $\mathrm{C}(58)-\mathrm{C}(57)-\mathrm{H}(57)$ & 107.5 \\
\hline
\end{tabular}




\begin{tabular}{|c|c|c|c|}
\hline $\mathrm{C}(59)-\mathrm{C}(57)-\mathrm{H}(57)$ & 107.5 & $\mathrm{C}(63)-\mathrm{C}(64)-\mathrm{H}(64)$ & 118.9 \\
\hline $\mathrm{C}(57)-\mathrm{C}(58)-\mathrm{H}(58 \mathrm{~A})$ & 109.5 & $C(66)-C(65)-C(70)$ & $120.6(5)$ \\
\hline $\mathrm{C}(57)-\mathrm{C}(58)-\mathrm{H}(58 \mathrm{~B})$ & 109.5 & $C(66)-C(65)-N(8)$ & $120.5(5)$ \\
\hline $\mathrm{H}(58 \mathrm{~A})-\mathrm{C}(58)-\mathrm{H}(58 \mathrm{~B})$ & 109.5 & $\mathrm{C}(70)-\mathrm{C}(65)-\mathrm{N}(8)$ & $118.9(5)$ \\
\hline $\mathrm{C}(57)-\mathrm{C}(58)-\mathrm{H}(58 \mathrm{C})$ & 109.5 & $C(67)-C(66)-C(65)$ & $118.6(5)$ \\
\hline $\mathrm{H}(58 \mathrm{~A})-\mathrm{C}(58)-\mathrm{H}(58 \mathrm{C})$ & 109.5 & $C(67)-C(66)-C(74)$ & $118.7(5)$ \\
\hline $\mathrm{H}(58 \mathrm{~B})-\mathrm{C}(58)-\mathrm{H}(58 \mathrm{C})$ & 109.5 & $C(65)-C(66)-C(74)$ & $122.7(5)$ \\
\hline $\mathrm{C}(57)-\mathrm{C}(59)-\mathrm{H}(59 \mathrm{~A})$ & 109.5 & $C(68)-C(67)-C(66)$ & $121.3(6)$ \\
\hline $\mathrm{C}(57)-\mathrm{C}(59)-\mathrm{H}(59 \mathrm{~B})$ & 109.5 & $\mathrm{C}(68)-\mathrm{C}(67)-\mathrm{H}(67)$ & 119.4 \\
\hline $\mathrm{H}(59 \mathrm{~A})-\mathrm{C}(59)-\mathrm{H}(59 \mathrm{~B})$ & 109.5 & $\mathrm{C}(66)-\mathrm{C}(67)-\mathrm{H}(67)$ & 119.4 \\
\hline $\mathrm{C}(57)-\mathrm{C}(59)-\mathrm{H}(59 \mathrm{C})$ & 109.5 & $C(67)-C(68)-C(69)$ & $119.4(6)$ \\
\hline $\mathrm{H}(59 \mathrm{~A})-\mathrm{C}(59)-\mathrm{H}(59 \mathrm{C})$ & 109.5 & $\mathrm{C}(67)-\mathrm{C}(68)-\mathrm{H}(68)$ & 120.3 \\
\hline $\mathrm{H}(59 \mathrm{~B})-\mathrm{C}(59)-\mathrm{H}(59 \mathrm{C})$ & 109.5 & $\mathrm{C}(69)-\mathrm{C}(68)-\mathrm{H}(68)$ & 120.3 \\
\hline$N(7)-C(60)-C(61)$ & $110.7(5)$ & $C(70)-C(69)-C(68)$ & $121.8(5)$ \\
\hline $\mathrm{N}(7)-\mathrm{C}(60)-\mathrm{H}(60)$ & 124.7 & $\mathrm{C}(70)-\mathrm{C}(69)-\mathrm{H}(69)$ & 119.1 \\
\hline $\mathrm{C}(61)-\mathrm{C}(60)-\mathrm{H}(60)$ & 124.7 & $\mathrm{C}(68)-\mathrm{C}(69)-\mathrm{H}(69)$ & 119.1 \\
\hline$C(62)-C(61)-C(60)$ & $107.3(5)$ & $C(69)-C(70)-C(65)$ & $118.2(5)$ \\
\hline $\mathrm{C}(62)-\mathrm{C}(61)-\mathrm{H}(61)$ & 126.4 & $C(69)-C(70)-C(71)$ & $120.5(5)$ \\
\hline $\mathrm{C}(60)-\mathrm{C}(61)-\mathrm{H}(61)$ & 126.4 & $C(65)-C(70)-C(71)$ & $121.1(5)$ \\
\hline$C(61)-C(62)-C(63)$ & $106.1(6)$ & $C(73)-C(71)-C(72)$ & $110.4(6)$ \\
\hline $\mathrm{C}(61)-\mathrm{C}(62)-\mathrm{H}(62)$ & 126.9 & $C(73)-C(71)-C(70)$ & $110.0(5)$ \\
\hline $\mathrm{C}(63)-\mathrm{C}(62)-\mathrm{H}(62)$ & 126.9 & $C(72)-C(71)-C(70)$ & $112.4(5)$ \\
\hline$N(7)-C(63)-C(64)$ & $118.7(5)$ & $\mathrm{C}(73)-\mathrm{C}(71)-\mathrm{H}(71)$ & 107.9 \\
\hline$N(7)-C(63)-C(62)$ & $109.8(5)$ & $\mathrm{C}(72)-\mathrm{C}(71)-\mathrm{H}(71)$ & 107.9 \\
\hline$C(64)-C(63)-C(62)$ & $131.4(6)$ & $\mathrm{C}(70)-\mathrm{C}(71)-\mathrm{H}(71)$ & 107.9 \\
\hline$N(8)-C(64)-C(63)$ & $122.2(5)$ & $\mathrm{C}(71)-\mathrm{C}(72)-\mathrm{H}(72 \mathrm{~A})$ & 109.5 \\
\hline $\mathrm{N}(8)-\mathrm{C}(64)-\mathrm{H}(64)$ & 118.9 & $\mathrm{C}(71)-\mathrm{C}(72)-\mathrm{H}(72 \mathrm{~B})$ & 109.5 \\
\hline
\end{tabular}




\begin{tabular}{|c|c|c|c|}
\hline $\mathrm{H}(72 \mathrm{~A})-\mathrm{C}(72)-\mathrm{H}(72 \mathrm{~B})$ & 109.5 & $\mathrm{H}(76 \mathrm{~B})-\mathrm{C}(76)-\mathrm{H}(76 \mathrm{C})$ & 109.5 \\
\hline $\mathrm{C}(71)-\mathrm{C}(72)-\mathrm{H}(72 \mathrm{C})$ & 109.5 & $\mathrm{Si}(5)-\mathrm{C}(77)-\mathrm{Lu}(2)$ & $129.9(3)$ \\
\hline $\mathrm{H}(72 \mathrm{~A})-\mathrm{C}(72)-\mathrm{H}(72 \mathrm{C})$ & 109.5 & $\mathrm{Si}(5)-\mathrm{C}(77)-\mathrm{H}(77 \mathrm{~A})$ & 104.8 \\
\hline $\mathrm{H}(72 \mathrm{~B})-\mathrm{C}(72)-\mathrm{H}(72 \mathrm{C})$ & 109.5 & $\mathrm{Lu}(2)-\mathrm{C}(77)-\mathrm{H}(77 \mathrm{~A})$ & 104.8 \\
\hline $\mathrm{C}(71)-\mathrm{C}(73)-\mathrm{H}(73 \mathrm{~A})$ & 109.5 & $\mathrm{Si}(5)-\mathrm{C}(77)-\mathrm{H}(77 \mathrm{~B})$ & 104.8 \\
\hline $\mathrm{C}(71)-\mathrm{C}(73)-\mathrm{H}(73 \mathrm{~B})$ & 109.5 & $\mathrm{Lu}(2)-\mathrm{C}(77)-\mathrm{H}(77 \mathrm{~B})$ & 104.8 \\
\hline $\mathrm{H}(73 \mathrm{~A})-\mathrm{C}(73)-\mathrm{H}(73 \mathrm{~B})$ & 109.5 & $\mathrm{H}(77 \mathrm{~A})-\mathrm{C}(77)-\mathrm{H}(77 \mathrm{~B})$ & 105.8 \\
\hline $\mathrm{C}(71)-\mathrm{C}(73)-\mathrm{H}(73 \mathrm{C})$ & 109.5 & $\mathrm{Si}(5)-\mathrm{C}(78)-\mathrm{H}(78 \mathrm{~A})$ & 109.5 \\
\hline $\mathrm{H}(73 \mathrm{~A})-\mathrm{C}(73)-\mathrm{H}(73 \mathrm{C})$ & 109.5 & $\mathrm{Si}(5)-\mathrm{C}(78)-\mathrm{H}(78 \mathrm{~B})$ & 109.5 \\
\hline $\mathrm{H}(73 \mathrm{~B})-\mathrm{C}(73)-\mathrm{H}(73 \mathrm{C})$ & 109.5 & $\mathrm{H}(78 \mathrm{~A})-\mathrm{C}(78)-\mathrm{H}(78 \mathrm{~B})$ & 109.5 \\
\hline$C(76)-C(74)-C(66)$ & $111.9(5)$ & $\mathrm{Si}(5)-\mathrm{C}(78)-\mathrm{H}(78 \mathrm{C})$ & 109.5 \\
\hline$C(76)-C(74)-C(75)$ & $110.1(5)$ & $\mathrm{H}(78 \mathrm{~A})-\mathrm{C}(78)-\mathrm{H}(78 \mathrm{C})$ & 109.5 \\
\hline$C(66)-C(74)-C(75)$ & $111.5(5)$ & $\mathrm{H}(78 \mathrm{~B})-\mathrm{C}(78)-\mathrm{H}(78 \mathrm{C})$ & 109.5 \\
\hline $\mathrm{C}(76)-\mathrm{C}(74)-\mathrm{H}(74)$ & 107.7 & $\mathrm{Si}(5)-\mathrm{C}(79)-\mathrm{H}(79 \mathrm{~A})$ & 109.5 \\
\hline $\mathrm{C}(66)-\mathrm{C}(74)-\mathrm{H}(74)$ & 107.7 & $\mathrm{Si}(5)-\mathrm{C}(79)-\mathrm{H}(79 \mathrm{~B})$ & 109.5 \\
\hline $\mathrm{C}(75)-\mathrm{C}(74)-\mathrm{H}(74)$ & 107.7 & $\mathrm{H}(79 \mathrm{~A})-\mathrm{C}(79)-\mathrm{H}(79 \mathrm{~B})$ & 109.5 \\
\hline $\mathrm{C}(74)-\mathrm{C}(75)-\mathrm{H}(75 \mathrm{~A})$ & 109.5 & $\mathrm{Si}(5)-\mathrm{C}(79)-\mathrm{H}(79 \mathrm{C})$ & 109.5 \\
\hline $\mathrm{C}(74)-\mathrm{C}(75)-\mathrm{H}(75 \mathrm{~B})$ & 109.5 & $\mathrm{H}(79 \mathrm{~A})-\mathrm{C}(79)-\mathrm{H}(79 \mathrm{C})$ & 109.5 \\
\hline $\mathrm{H}(75 \mathrm{~A})-\mathrm{C}(75)-\mathrm{H}(75 \mathrm{~B})$ & 109.5 & $\mathrm{H}(79 \mathrm{~B})-\mathrm{C}(79)-\mathrm{H}(79 \mathrm{C})$ & 109.5 \\
\hline $\mathrm{C}(74)-\mathrm{C}(75)-\mathrm{H}(75 \mathrm{C})$ & 109.5 & $\mathrm{Si}(5)-\mathrm{C}(80)-\mathrm{H}(80 \mathrm{~A})$ & 109.5 \\
\hline $\mathrm{H}(75 \mathrm{~A})-\mathrm{C}(75)-\mathrm{H}(75 \mathrm{C})$ & 109.5 & $\mathrm{Si}(5)-\mathrm{C}(80)-\mathrm{H}(80 \mathrm{~B})$ & 109.5 \\
\hline $\mathrm{H}(75 \mathrm{~B})-\mathrm{C}(75)-\mathrm{H}(75 \mathrm{C})$ & 109.5 & $\mathrm{H}(80 \mathrm{~A})-\mathrm{C}(80)-\mathrm{H}(80 \mathrm{~B})$ & 109.5 \\
\hline $\mathrm{C}(74)-\mathrm{C}(76)-\mathrm{H}(76 \mathrm{~A})$ & 109.5 & $\mathrm{Si}(5)-\mathrm{C}(80)-\mathrm{H}(80 \mathrm{C})$ & 109.5 \\
\hline $\mathrm{C}(74)-\mathrm{C}(76)-\mathrm{H}(76 \mathrm{~B})$ & 109.5 & $\mathrm{H}(80 \mathrm{~A})-\mathrm{C}(80)-\mathrm{H}(80 \mathrm{C})$ & 109.5 \\
\hline $\mathrm{H}(76 \mathrm{~A})-\mathrm{C}(76)-\mathrm{H}(76 \mathrm{~B})$ & 109.5 & $\mathrm{H}(80 \mathrm{~B})-\mathrm{C}(80)-\mathrm{H}(80 \mathrm{C})$ & 109.5 \\
\hline $\mathrm{C}(74)-\mathrm{C}(76)-\mathrm{H}(76 \mathrm{C})$ & 109.5 & $\mathrm{O}(2)-\mathrm{C}(81)-\mathrm{C}(82)$ & $105.6(5)$ \\
\hline $\mathrm{H}(76 \mathrm{~A})-\mathrm{C}(76)-\mathrm{H}(76 \mathrm{C})$ & 109.5 & $\mathrm{O}(2)-\mathrm{C}(81)-\mathrm{H}(81 \mathrm{~A})$ & 110.6 \\
\hline
\end{tabular}




\begin{tabular}{lrlr}
$\mathrm{C}(82)-\mathrm{C}(81)-\mathrm{H}(81 \mathrm{~A})$ & 110.6 & $\mathrm{C}(82)-\mathrm{C}(83)-\mathrm{H}(83)$ & 125.6 \\
$\mathrm{O}(2)-\mathrm{C}(81)-\mathrm{H}(81 \mathrm{~B})$ & 110.6 & $\mathrm{C}(84)-\mathrm{C}(83)-\mathrm{H}(83)$ & 125.6 \\
$\mathrm{C}(82)-\mathrm{C}(81)-\mathrm{H}(81 \mathrm{~B})$ & 110.6 & $\mathrm{O}(2)-\mathrm{C}(84)-\mathrm{C}(83)$ & $105.4(5)$ \\
$\mathrm{H}(81 \mathrm{~A})-\mathrm{C}(81)-\mathrm{H}(81 \mathrm{~B})$ & 108.8 & $\mathrm{O}(2)-\mathrm{C}(84)-\mathrm{H}(84 \mathrm{~A})$ & 110.7 \\
$\mathrm{C}(83)-\mathrm{C}(82)-\mathrm{C}(81)$ & $110.9(6)$ & $\mathrm{C}(83)-\mathrm{C}(84)-\mathrm{H}(84 \mathrm{~A})$ & 110.7 \\
$\mathrm{C}(83)-\mathrm{C}(82)-\mathrm{H}(82)$ & 124.5 & $\mathrm{O}(2)-\mathrm{C}(84)-\mathrm{H}(84 \mathrm{~B})$ & 110.7 \\
$\mathrm{C}(81)-\mathrm{C}(82)-\mathrm{H}(82)$ & 124.5 & $\mathrm{C}(83)-\mathrm{C}(84)-\mathrm{H}(84 \mathrm{~B})$ & 108.8 \\
$\mathrm{C}(82)-\mathrm{C}(83)-\mathrm{C}(84)$ & $108.7(6)$ & $\mathrm{H}(84 \mathrm{~A})-\mathrm{C}(84)-\mathrm{H}(84 \mathrm{~B})$ & \\
& & & \\
\hline
\end{tabular}

\#---End of complex 2a 
Stable 14. Crystal data and structure refinement for $3 a$

Identification code

Empirical formula

Formula weight

Temperature

Wavelength

Crystal system

Space group

Unit cell dimensions

Volume

Z

Density (calculated)

Absorption coefficient

$\mathrm{F}(000)$

Crystal size

Theta range for data collection

Index ranges

Reflections collected

Independent reflections

Completeness to theta $=26.04^{\circ}$

Absorption correction

Max. and min. transmission

Refinement method

Data / restraints / parameters q201m

C30 H66 N4 Si4 Y2

773.05

187(2) K

$0.71073 \AA$

Triclinic

P-1

$$
\begin{array}{ll}
\mathrm{a}=9.4442(8) \AA & \alpha=77.9230(10)^{\circ} . \\
\mathrm{b}=10.4910(9) \AA & \beta=84.3380(10)^{\circ} . \\
\mathrm{c}=11.4717(10) \AA & \gamma=65.7770(10)^{\circ} .
\end{array}
$$

$1013.49(15) \AA^{3}$

1

$1.267 \mathrm{Mg} / \mathrm{m}^{3}$

$2.990 \mathrm{~mm}^{-1}$

408

$0.18 \times 0.15 \times 0.13 \mathrm{~mm}^{3}$

1.82 to $26.04^{\circ}$.

$-11<=\mathrm{h}<=4,-12<=\mathrm{k}<=12,-14<=\mathrm{l}<=14$

5714

$3900[\mathrm{R}($ int $)=0.0876]$

$97.6 \%$

Semi-empirical from equivalents

0.6972 and 0.6151

Full-matrix least-squares on $\mathrm{F}^{2}$

$3900 / 0 / 189$ 
Goodness-of-fit on $\mathrm{F}^{2}$

Final $\mathrm{R}$ indices $[\mathrm{I}>2 \operatorname{sigma}(\mathrm{I})]$

$\mathrm{R}$ indices (all data)

Largest diff. peak and hole
0.996

$\mathrm{R} 1=0.0389, \mathrm{wR} 2=0.0966$

$\mathrm{R} 1=0.0453, \mathrm{wR} 2=0.0987$

0.961 and $-0.933 \mathrm{e}^{-3}$ 
Stable 15. Atomic coordinates ( $\left.\begin{array}{ll}x & 10^{4}\end{array}\right)$ and equivalent isotropic displacement parameters $\left(\AA^{2} \times 10^{3}\right)$ for $3 a$. $U(e q)$ is defined as one third of the trace of the orthogonalized Uij tensor.

\begin{tabular}{|c|c|c|c|c|}
\hline & $\mathrm{x}$ & $\mathrm{y}$ & $\mathrm{z}$ & $\mathrm{U}(\mathrm{eq})$ \\
\hline $\mathrm{Y}$ & $1162(1)$ & $895(1)$ & $8635(1)$ & $24(1)$ \\
\hline $\mathrm{Si}(1)$ & 2161(1) & $3929(1)$ & 6829(1) & $30(1)$ \\
\hline $\mathrm{Si}(2)$ & $4761(1)$ & $-1880(1)$ & $7644(1)$ & $32(1)$ \\
\hline $\mathrm{N}(1)$ & $-313(3)$ & 1066(3) & $10807(2)$ & $24(1)$ \\
\hline $\mathrm{N}(2)$ & $1229(3)$ & $-1267(3)$ & $12532(2)$ & $29(1)$ \\
\hline $\mathrm{C}(1)$ & 1206(4) & $776(3)$ & $11028(2)$ & $25(1)$ \\
\hline $\mathrm{C}(2)$ & 1562(4) & 1940(4) & 10517(3) & $30(1)$ \\
\hline $\mathrm{C}(3)$ & $189(4)$ & 2999(3) & 9967(3) & $32(1)$ \\
\hline $\mathrm{C}(4)$ & $-926(4)$ & 2437(3) & $10167(2)$ & $27(1)$ \\
\hline $\mathrm{C}(5)$ & 2193(4) & $-656(3)$ & $11710(3)$ & $29(1)$ \\
\hline$C(6)$ & $742(4)$ & $-537(4)$ & 13566(3) & $39(1)$ \\
\hline$C(7)$ & $2184(5)$ & $-2782(4)$ & 12976(3) & $47(1)$ \\
\hline $\mathrm{C}(8)$ & $3835(4)$ & $-771(4)$ & 8781(3) & $39(1)$ \\
\hline $\mathrm{C}(9)$ & $1710(5)$ & $2366(4)$ & 6902(3) & $39(1)$ \\
\hline$C(10)$ & $5922(5)$ & $-3770(4)$ & 8344(4) & $57(1)$ \\
\hline $\mathrm{C}(11)$ & $3289(5)$ & $-1940(6)$ & $6702(4)$ & $57(1)$ \\
\hline $\mathrm{C}(12)$ & $6070(5)$ & $-1227(4)$ & 6593(3) & $46(1)$ \\
\hline $\mathrm{C}(13)$ & $3317(5)$ & $3768(5)$ & $8133(3)$ & $47(1)$ \\
\hline$C(14)$ & $374(5)$ & $5595(4)$ & 6753(4) & $55(1)$ \\
\hline$C(15)$ & $3379(5)$ & $4187(5)$ & $5483(3)$ & $47(1)$ \\
\hline
\end{tabular}


Stable 16. Anisotropic displacement parameters $\left(\AA^{2} x 1^{3}\right)$ for 3a. The anisotropic displacement factor exponent takes the form: $-2 \quad 2_{[} h^{2} a * 2 U^{11}+\ldots$ $\left.+2 \mathrm{~h} \mathrm{k} \mathrm{a*} \mathbf{b}^{*} \mathbf{U}^{12}\right]$

\begin{tabular}{|c|c|c|c|c|c|c|}
\hline & U11 & $\mathrm{U} 22$ & U33 & $\mathrm{U} 23$ & U13 & U12 \\
\hline $\mathrm{Y}$ & $32(1)$ & $22(1)$ & $22(1)$ & $-5(1)$ & $0(1)$ & $-15(1)$ \\
\hline $\mathrm{Si}(1)$ & $34(1)$ & $28(1)$ & $31(1)$ & $1(1)$ & $-1(1)$ & $-17(1)$ \\
\hline $\mathrm{Si}(2)$ & $32(1)$ & $35(1)$ & $35(1)$ & $-14(1)$ & $2(1)$ & $-14(1)$ \\
\hline $\mathrm{N}(1)$ & $29(1)$ & $22(1)$ & $22(1)$ & $-6(1)$ & 1(1) & $-12(1)$ \\
\hline $\mathrm{N}(2)$ & $35(2)$ & $24(1)$ & $26(1)$ & $0(1)$ & $-6(1)$ & $-12(1)$ \\
\hline $\mathrm{C}(1)$ & $33(2)$ & $27(2)$ & $20(1)$ & $-8(1)$ & 1(1) & $-16(1)$ \\
\hline $\mathrm{C}(2)$ & $41(2)$ & $34(2)$ & $27(2)$ & $-10(1)$ & $3(1)$ & $-25(2)$ \\
\hline$C(3)$ & $53(2)$ & $20(2)$ & $30(2)$ & $-6(1)$ & $2(2)$ & $-22(2)$ \\
\hline $\mathrm{C}(4)$ & $31(2)$ & $20(2)$ & $27(2)$ & $-5(1)$ & $-2(1)$ & $-8(1)$ \\
\hline $\mathrm{C}(5)$ & $31(2)$ & $32(2)$ & $26(2)$ & $-4(1)$ & $-3(1)$ & $-14(2)$ \\
\hline$C(6)$ & $45(2)$ & $48(2)$ & $27(2)$ & $-6(2)$ & $-2(2)$ & $-23(2)$ \\
\hline$C(7)$ & $51(2)$ & $30(2)$ & $49(2)$ & $8(2)$ & $-14(2)$ & $-10(2)$ \\
\hline $\mathrm{C}(8)$ & $37(2)$ & $43(2)$ & $36(2)$ & $-12(2)$ & $0(2)$ & $-14(2)$ \\
\hline $\mathrm{C}(9)$ & $53(2)$ & $42(2)$ & $29(2)$ & $-4(2)$ & $2(2)$ & $-28(2)$ \\
\hline$C(10)$ & $75(3)$ & $36(2)$ & $62(2)$ & $-11(2)$ & $-5(2)$ & $-23(2)$ \\
\hline$C(11)$ & $48(2)$ & $83(3)$ & $53(2)$ & $-37(2)$ & $4(2)$ & $-28(3)$ \\
\hline $\mathrm{C}(12)$ & $55(2)$ & $50(2)$ & $44(2)$ & $-17(2)$ & $7(2)$ & $-29(2)$ \\
\hline $\mathrm{C}(13)$ & $53(2)$ & $58(3)$ & $40(2)$ & $-5(2)$ & $-3(2)$ & $-36(2)$ \\
\hline$C(14)$ & $48(2)$ & $42(2)$ & $65(3)$ & $-7(2)$ & $-5(2)$ & $-8(2)$ \\
\hline$C(15)$ & $60(3)$ & $60(3)$ & $35(2)$ & $-4(2)$ & $6(2)$ & $-41(2)$ \\
\hline
\end{tabular}


Stable 17. Bond lengths $[\AA ̊]$ and angles $\left[{ }^{\circ}\right]$ for $3 a$.

\begin{tabular}{|c|c|c|c|}
\hline $\mathrm{Y}-\mathrm{C}(9)$ & $2.402(3)$ & $C(2)-C(3)$ & $1.414(5)$ \\
\hline $\mathrm{Y}-\mathrm{C}(8)$ & $2.408(4)$ & $\mathrm{C}(2)-\mathrm{H}(2)$ & 0.9300 \\
\hline $\mathrm{Y}-\mathrm{N}(1) \# 1$ & $2.446(2)$ & $C(3)-C(4)$ & $1.384(4)$ \\
\hline $\mathrm{Y}-\mathrm{N}(2) \# 1$ & $2.588(3)$ & $\mathrm{C}(3)-\mathrm{H}(3)$ & 0.9300 \\
\hline $\mathrm{Y}-\mathrm{C}(1)$ & $2.725(3)$ & $\mathrm{C}(4)-\mathrm{H}(4)$ & 0.9300 \\
\hline $\mathrm{Y}-\mathrm{N}(1)$ & $2.735(2)$ & $\mathrm{C}(5)-\mathrm{H}(5 \mathrm{~A})$ & 0.9700 \\
\hline $\mathrm{Y}-\mathrm{C}(4)$ & $2.735(3)$ & $\mathrm{C}(5)-\mathrm{H}(5 \mathrm{~B})$ & 0.9700 \\
\hline $\mathrm{Y}-\mathrm{C}(2)$ & $2.738(3)$ & $\mathrm{C}(6)-\mathrm{H}(6 \mathrm{~A})$ & 0.9600 \\
\hline $\mathrm{Y}-\mathrm{C}(3)$ & $2.743(3)$ & $\mathrm{C}(6)-\mathrm{H}(6 \mathrm{~B})$ & 0.9600 \\
\hline $\mathrm{Si}(1)-\mathrm{C}(9)$ & $1.840(4)$ & $\mathrm{C}(6)-\mathrm{H}(6 \mathrm{C})$ & 0.9600 \\
\hline $\operatorname{Si}(1)-C(14)$ & $1.859(4)$ & $\mathrm{C}(7)-\mathrm{H}(7 \mathrm{~A})$ & 0.9600 \\
\hline $\mathrm{Si}(1)-\mathrm{C}(15)$ & $1.873(3)$ & $\mathrm{C}(7)-\mathrm{H}(7 \mathrm{~B})$ & 0.9600 \\
\hline $\mathrm{Si}(1)-\mathrm{C}(13)$ & $1.877(4)$ & $\mathrm{C}(7)-\mathrm{H}(7 \mathrm{C})$ & 0.9600 \\
\hline $\operatorname{Si}(2)-C(8)$ & $1.842(4)$ & $\mathrm{C}(8)-\mathrm{H}(8 \mathrm{~A})$ & 0.9700 \\
\hline $\mathrm{Si}(2)-\mathrm{C}(11)$ & $1.871(4)$ & $\mathrm{C}(8)-\mathrm{H}(8 \mathrm{~B})$ & 0.9700 \\
\hline $\operatorname{Si}(2)-C(10)$ & $1.871(4)$ & $\mathrm{C}(9)-\mathrm{H}(9 \mathrm{~A})$ & 0.9700 \\
\hline $\operatorname{Si}(2)-C(12)$ & $1.873(4)$ & $\mathrm{C}(9)-\mathrm{H}(9 \mathrm{~B})$ & 0.9700 \\
\hline $\mathrm{N}(1)-\mathrm{C}(1)$ & $1.377(4)$ & $\mathrm{C}(10)-\mathrm{H}(10 \mathrm{~A})$ & 0.9600 \\
\hline $\mathrm{N}(1)-\mathrm{C}(4)$ & $1.379(4)$ & $\mathrm{C}(10)-\mathrm{H}(10 \mathrm{~B})$ & 0.9600 \\
\hline $\mathrm{N}(1)-\mathrm{Y} \# 1$ & $2.446(2)$ & $\mathrm{C}(10)-\mathrm{H}(10 \mathrm{C})$ & 0.9600 \\
\hline $\mathrm{N}(2)-\mathrm{C}(7)$ & $1.475(4)$ & $\mathrm{C}(11)-\mathrm{H}(11 \mathrm{~A})$ & 0.9600 \\
\hline $\mathrm{N}(2)-\mathrm{C}(5)$ & $1.478(4)$ & $\mathrm{C}(11)-\mathrm{H}(11 \mathrm{~B})$ & 0.9600 \\
\hline $\mathrm{N}(2)-\mathrm{C}(6)$ & $1.479(4)$ & $\mathrm{C}(11)-\mathrm{H}(11 \mathrm{C})$ & 0.9600 \\
\hline $\mathrm{N}(2)-\mathrm{Y} \# 1$ & $2.588(3)$ & $\mathrm{C}(12)-\mathrm{H}(12 \mathrm{~A})$ & 0.9600 \\
\hline$C(1)-C(2)$ & $1.395(4)$ & $\mathrm{C}(12)-\mathrm{H}(12 \mathrm{~B})$ & 0.9600 \\
\hline$C(1)-C(5)$ & $1.497(4)$ & $\mathrm{C}(12)-\mathrm{H}(12 \mathrm{C})$ & 0.9600 \\
\hline
\end{tabular}




\begin{tabular}{|c|c|c|c|}
\hline $\mathrm{C}(13)-\mathrm{H}(13 \mathrm{~A})$ & 0.9600 & $\mathrm{C}(14)-\mathrm{H}(14 \mathrm{C})$ & 0.9600 \\
\hline $\mathrm{C}(13)-\mathrm{H}(13 \mathrm{~B})$ & 0.9600 & $\mathrm{C}(15)-\mathrm{H}(15 \mathrm{~A})$ & 0.9600 \\
\hline $\mathrm{C}(13)-\mathrm{H}(13 \mathrm{C})$ & 0.9600 & $\mathrm{C}(15)-\mathrm{H}(15 \mathrm{~B})$ & 0.9600 \\
\hline $\mathrm{C}(14)-\mathrm{H}(14 \mathrm{~A})$ & 0.9600 & $\mathrm{C}(15)-\mathrm{H}(15 \mathrm{C})$ & 0.9600 \\
\hline $\mathrm{C}(14)-\mathrm{H}(14 \mathrm{~B})$ & 0.9600 & & \\
\hline$C(9)-Y-C(8)$ & $91.99(13)$ & $\mathrm{C}(8)-\mathrm{Y}-\mathrm{C}(2)$ & $89.50(11)$ \\
\hline $\mathrm{C}(9)-\mathrm{Y}-\mathrm{N}(1) \# 1$ & $140.83(10)$ & $\mathrm{N}(1) \# 1-\mathrm{Y}-\mathrm{C}(2)$ & $114.56(8)$ \\
\hline $\mathrm{C}(8)-\mathrm{Y}-\mathrm{N}(1) \# 1$ & $90.35(11)$ & $\mathrm{N}(2) \# 1-\mathrm{Y}-\mathrm{C}(2)$ & $134.12(10)$ \\
\hline $\mathrm{C}(9)-\mathrm{Y}-\mathrm{N}(2) \# 1$ & $85.15(10)$ & $C(1)-Y-C(2)$ & $29.59(9)$ \\
\hline $\mathrm{C}(8)-\mathrm{Y}-\mathrm{N}(2) \# 1$ & $135.58(11)$ & $\mathrm{N}(1)-\mathrm{Y}-\mathrm{C}(2)$ & $49.13(8)$ \\
\hline $\mathrm{N}(1) \# 1-\mathrm{Y}-\mathrm{N}(2) \# 1$ & $66.55(8)$ & $C(4)-Y-C(2)$ & $48.48(10)$ \\
\hline$C(9)-Y-C(1)$ & $134.11(11)$ & $C(9)-Y-C(3)$ & $93.36(11)$ \\
\hline$C(8)-Y-C(1)$ & $87.38(10)$ & $\mathrm{C}(8)-\mathrm{Y}-\mathrm{C}(3)$ & $117.93(11)$ \\
\hline N(1)\#1-Y-C(1) & $85.06(8)$ & $\mathrm{N}(1) \# 1-\mathrm{Y}-\mathrm{C}(3)$ & $119.62(8)$ \\
\hline $\mathrm{N}(2) \# 1-\mathrm{Y}-\mathrm{C}(1)$ & $125.07(9)$ & $\mathrm{N}(2) \# 1-\mathrm{Y}-\mathrm{C}(3)$ & $106.49(10)$ \\
\hline $\mathrm{C}(9)-\mathrm{Y}-\mathrm{N}(1)$ & $141.09(11)$ & $C(1)-Y-C(3)$ & $48.51(9)$ \\
\hline $\mathrm{C}(8)-\mathrm{Y}-\mathrm{N}(1)$ & $112.65(9)$ & $\mathrm{N}(1)-\mathrm{Y}-\mathrm{C}(3)$ & $48.93(8)$ \\
\hline $\mathrm{N}(1) \# 1-\mathrm{Y}-\mathrm{N}(1)$ & $71.47(9)$ & $C(4)-Y-C(3)$ & $29.26(9)$ \\
\hline $\mathrm{N}(2) \# 1-\mathrm{Y}-\mathrm{N}(1)$ & $95.90(8)$ & $\mathrm{C}(2)-\mathrm{Y}-\mathrm{C}(3)$ & $29.91(10)$ \\
\hline$C(1)-Y-N(1)$ & $29.21(8)$ & $\mathrm{C}(9)-\mathrm{Si}(1)-\mathrm{C}(14)$ & $111.85(19)$ \\
\hline$C(9)-Y-C(4)$ & $112.85(11)$ & $\mathrm{C}(9)-\mathrm{Si}(1)-\mathrm{C}(15)$ & $111.71(18)$ \\
\hline$C(8)-Y-C(4)$ & $134.34(10)$ & $\mathrm{C}(14)-\mathrm{Si}(1)-\mathrm{C}(15)$ & $107.00(19)$ \\
\hline $\mathrm{N}(1) \# 1-\mathrm{Y}-\mathrm{C}(4)$ & $92.51(9)$ & $\mathrm{C}(9)-\mathrm{Si}(1)-\mathrm{C}(13)$ & $112.76(16)$ \\
\hline $\mathrm{N}(2) \# 1-\mathrm{Y}-\mathrm{C}(4)$ & $86.14(9)$ & $C(14)-\operatorname{Si}(1)-C(13)$ & $108.0(2)$ \\
\hline$C(1)-Y-C(4)$ & $47.62(9)$ & $\mathrm{C}(15)-\mathrm{Si}(1)-\mathrm{C}(13)$ & $105.12(17)$ \\
\hline$N(1)-Y-C(4)$ & $29.21(8)$ & $\mathrm{C}(8)-\mathrm{Si}(2)-\mathrm{C}(11)$ & $111.68(18)$ \\
\hline $\mathrm{C}(9)-\mathrm{Y}-\mathrm{C}(2)$ & $104.56(11)$ & $C(8)-\operatorname{Si}(2)-C(10)$ & $111.40(18)$ \\
\hline
\end{tabular}




\begin{tabular}{|c|c|c|c|}
\hline$C(11)-\operatorname{Si}(2)-C(10)$ & $105.8(2)$ & $\mathrm{Y}-\mathrm{C}(2)-\mathrm{H}(2)$ & 115.6 \\
\hline $\mathrm{C}(8)-\mathrm{Si}(2)-\mathrm{C}(12)$ & $113.16(17)$ & $C(4)-C(3)-C(2)$ & $106.9(3)$ \\
\hline $\mathrm{C}(11)-\mathrm{Si}(2)-\mathrm{C}(12)$ & $106.35(19)$ & $C(4)-C(3)-Y$ & $75.08(18)$ \\
\hline $\mathrm{C}(10)-\mathrm{Si}(2)-\mathrm{C}(12)$ & $108.0(2)$ & $\mathrm{C}(2)-\mathrm{C}(3)-\mathrm{Y}$ & $74.86(19)$ \\
\hline $\mathrm{C}(1)-\mathrm{N}(1)-\mathrm{C}(4)$ & $106.2(2)$ & $\mathrm{C}(4)-\mathrm{C}(3)-\mathrm{H}(3)$ & 126.6 \\
\hline $\mathrm{C}(1)-\mathrm{N}(1)-\mathrm{Y} \# 1$ & 117.44(19) & $\mathrm{C}(2)-\mathrm{C}(3)-\mathrm{H}(3)$ & 126.6 \\
\hline$C(4)-N(1)-Y \# 1$ & $135.8(2)$ & $\mathrm{Y}-\mathrm{C}(3)-\mathrm{H}(3)$ & 115.8 \\
\hline$C(1)-N(1)-Y$ & $75.01(15)$ & $\mathrm{N}(1)-\mathrm{C}(4)-\mathrm{C}(3)$ & $110.4(3)$ \\
\hline$C(4)-N(1)-Y$ & $75.43(16)$ & $N(1)-C(4)-Y$ & $75.37(17)$ \\
\hline $\mathrm{Y} \# 1-\mathrm{N}(1)-\mathrm{Y}$ & $108.53(9)$ & $C(3)-C(4)-Y$ & $75.67(18)$ \\
\hline$C(7)-N(2)-C(5)$ & $108.5(3)$ & $\mathrm{N}(1)-\mathrm{C}(4)-\mathrm{H}(4)$ & 124.8 \\
\hline$C(7)-N(2)-C(6)$ & $108.0(3)$ & $\mathrm{C}(3)-\mathrm{C}(4)-\mathrm{H}(4)$ & 124.8 \\
\hline$C(5)-N(2)-C(6)$ & $109.3(2)$ & $\mathrm{Y}-\mathrm{C}(4)-\mathrm{H}(4)$ & 116.0 \\
\hline$C(7)-N(2)-Y \# 1$ & $112.6(2)$ & $\mathrm{N}(2)-\mathrm{C}(5)-\mathrm{C}(1)$ & $110.7(3)$ \\
\hline $\mathrm{C}(5)-\mathrm{N}(2)-\mathrm{Y} \# 1$ & $107.62(16)$ & $\mathrm{N}(2)-\mathrm{C}(5)-\mathrm{H}(5 \mathrm{~A})$ & 109.5 \\
\hline$C(6)-N(2)-Y \# 1$ & $110.7(2)$ & $\mathrm{C}(1)-\mathrm{C}(5)-\mathrm{H}(5 \mathrm{~A})$ & 109.5 \\
\hline $\mathrm{N}(1)-\mathrm{C}(1)-\mathrm{C}(2)$ & $110.3(3)$ & $\mathrm{N}(2)-\mathrm{C}(5)-\mathrm{H}(5 \mathrm{~B})$ & 109.5 \\
\hline $\mathrm{N}(1)-\mathrm{C}(1)-\mathrm{C}(5)$ & $118.9(3)$ & $\mathrm{C}(1)-\mathrm{C}(5)-\mathrm{H}(5 \mathrm{~B})$ & 109.5 \\
\hline$C(2)-C(1)-C(5)$ & $130.7(3)$ & $\mathrm{H}(5 \mathrm{~A})-\mathrm{C}(5)-\mathrm{H}(5 \mathrm{~B})$ & 108.1 \\
\hline$N(1)-C(1)-Y$ & $75.78(15)$ & $\mathrm{N}(2)-\mathrm{C}(6)-\mathrm{H}(6 \mathrm{~A})$ & 109.5 \\
\hline$C(2)-C(1)-Y$ & $75.72(16)$ & $\mathrm{N}(2)-\mathrm{C}(6)-\mathrm{H}(6 \mathrm{~B})$ & 109.5 \\
\hline$C(5)-C(1)-Y$ & $114.3(2)$ & $H(6 A)-C(6)-H(6 B)$ & 109.5 \\
\hline$C(1)-C(2)-C(3)$ & $106.2(3)$ & $\mathrm{N}(2)-\mathrm{C}(6)-\mathrm{H}(6 \mathrm{C})$ & 109.5 \\
\hline$C(1)-C(2)-Y$ & $74.69(17)$ & $H(6 A)-C(6)-H(6 C)$ & 109.5 \\
\hline$C(3)-C(2)-Y$ & $75.23(17)$ & $\mathrm{H}(6 \mathrm{~B})-\mathrm{C}(6)-\mathrm{H}(6 \mathrm{C})$ & 109.5 \\
\hline $\mathrm{C}(1)-\mathrm{C}(2)-\mathrm{H}(2)$ & 126.9 & $\mathrm{~N}(2)-\mathrm{C}(7)-\mathrm{H}(7 \mathrm{~A})$ & 109.5 \\
\hline $\mathrm{C}(3)-\mathrm{C}(2)-\mathrm{H}(2)$ & 126.9 & $\mathrm{~N}(2)-\mathrm{C}(7)-\mathrm{H}(7 \mathrm{~B})$ & 109.5 \\
\hline
\end{tabular}




\begin{tabular}{|c|c|c|c|}
\hline $\mathrm{H}(7 \mathrm{~A})-\mathrm{C}(7)-\mathrm{H}(7 \mathrm{~B})$ & 109.5 & $\mathrm{H}(11 \mathrm{~A})-\mathrm{C}(11)-\mathrm{H}(11 \mathrm{C})$ & 109.5 \\
\hline $\mathrm{N}(2)-\mathrm{C}(7)-\mathrm{H}(7 \mathrm{C})$ & 109.5 & $\mathrm{H}(11 \mathrm{~B})-\mathrm{C}(11)-\mathrm{H}(11 \mathrm{C})$ & 109.5 \\
\hline $\mathrm{H}(7 \mathrm{~A})-\mathrm{C}(7)-\mathrm{H}(7 \mathrm{C})$ & 109.5 & $\mathrm{Si}(2)-\mathrm{C}(12)-\mathrm{H}(12 \mathrm{~A})$ & 109.5 \\
\hline $\mathrm{H}(7 \mathrm{~B})-\mathrm{C}(7)-\mathrm{H}(7 \mathrm{C})$ & 109.5 & $\mathrm{Si}(2)-\mathrm{C}(12)-\mathrm{H}(12 \mathrm{~B})$ & 109.5 \\
\hline $\operatorname{Si}(2)-C(8)-Y$ & $121.50(16)$ & $\mathrm{H}(12 \mathrm{~A})-\mathrm{C}(12)-\mathrm{H}(12 \mathrm{~B})$ & 109.5 \\
\hline $\mathrm{Si}(2)-\mathrm{C}(8)-\mathrm{H}(8 \mathrm{~A})$ & 107.0 & $\mathrm{Si}(2)-\mathrm{C}(12)-\mathrm{H}(12 \mathrm{C})$ & 109.5 \\
\hline $\mathrm{Y}-\mathrm{C}(8)-\mathrm{H}(8 \mathrm{~A})$ & 107.0 & $\mathrm{H}(12 \mathrm{~A})-\mathrm{C}(12)-\mathrm{H}(12 \mathrm{C})$ & 109.5 \\
\hline $\mathrm{Si}(2)-\mathrm{C}(8)-\mathrm{H}(8 \mathrm{~B})$ & 107.0 & $\mathrm{H}(12 \mathrm{~B})-\mathrm{C}(12)-\mathrm{H}(12 \mathrm{C})$ & 109.5 \\
\hline $\mathrm{Y}-\mathrm{C}(8)-\mathrm{H}(8 \mathrm{~B})$ & 107.0 & $\mathrm{Si}(1)-\mathrm{C}(13)-\mathrm{H}(13 \mathrm{~A})$ & 109.5 \\
\hline $\mathrm{H}(8 \mathrm{~A})-\mathrm{C}(8)-\mathrm{H}(8 \mathrm{~B})$ & 106.7 & $\mathrm{Si}(1)-\mathrm{C}(13)-\mathrm{H}(13 \mathrm{~B})$ & 109.5 \\
\hline $\operatorname{Si}(1)-C(9)-Y$ & $128.30(17)$ & $\mathrm{H}(13 \mathrm{~A})-\mathrm{C}(13)-\mathrm{H}(13 \mathrm{~B})$ & 109.5 \\
\hline $\mathrm{Si}(1)-\mathrm{C}(9)-\mathrm{H}(9 \mathrm{~A})$ & 105.2 & $\mathrm{Si}(1)-\mathrm{C}(13)-\mathrm{H}(13 \mathrm{C})$ & 109.5 \\
\hline $\mathrm{Y}-\mathrm{C}(9)-\mathrm{H}(9 \mathrm{~A})$ & 105.2 & $\mathrm{H}(13 \mathrm{~A})-\mathrm{C}(13)-\mathrm{H}(13 \mathrm{C})$ & 109.5 \\
\hline $\mathrm{Si}(1)-\mathrm{C}(9)-\mathrm{H}(9 \mathrm{~B})$ & 105.2 & $\mathrm{H}(13 \mathrm{~B})-\mathrm{C}(13)-\mathrm{H}(13 \mathrm{C})$ & 109.5 \\
\hline $\mathrm{Y}-\mathrm{C}(9)-\mathrm{H}(9 \mathrm{~B})$ & 105.2 & $\mathrm{Si}(1)-\mathrm{C}(14)-\mathrm{H}(14 \mathrm{~A})$ & 109.5 \\
\hline $\mathrm{H}(9 \mathrm{~A})-\mathrm{C}(9)-\mathrm{H}(9 \mathrm{~B})$ & 105.9 & $\mathrm{Si}(1)-\mathrm{C}(14)-\mathrm{H}(14 \mathrm{~B})$ & 109.5 \\
\hline $\mathrm{Si}(2)-\mathrm{C}(10)-\mathrm{H}(10 \mathrm{~A})$ & 109.5 & $\mathrm{H}(14 \mathrm{~A})-\mathrm{C}(14)-\mathrm{H}(14 \mathrm{~B})$ & 109.5 \\
\hline $\mathrm{Si}(2)-\mathrm{C}(10)-\mathrm{H}(10 \mathrm{~B})$ & 109.5 & $\mathrm{Si}(1)-\mathrm{C}(14)-\mathrm{H}(14 \mathrm{C})$ & 109.5 \\
\hline $\mathrm{H}(10 \mathrm{~A})-\mathrm{C}(10)-\mathrm{H}(10 \mathrm{~B})$ & 109.5 & $\mathrm{H}(14 \mathrm{~A})-\mathrm{C}(14)-\mathrm{H}(14 \mathrm{C})$ & 109.5 \\
\hline $\mathrm{Si}(2)-\mathrm{C}(10)-\mathrm{H}(10 \mathrm{C})$ & 109.5 & $\mathrm{H}(14 \mathrm{~B})-\mathrm{C}(14)-\mathrm{H}(14 \mathrm{C})$ & 109.5 \\
\hline $\mathrm{H}(10 \mathrm{~A})-\mathrm{C}(10)-\mathrm{H}(10 \mathrm{C})$ & 109.5 & $\mathrm{Si}(1)-\mathrm{C}(15)-\mathrm{H}(15 \mathrm{~A})$ & 109.5 \\
\hline $\mathrm{H}(10 \mathrm{~B})-\mathrm{C}(10)-\mathrm{H}(10 \mathrm{C})$ & 109.5 & $\mathrm{Si}(1)-\mathrm{C}(15)-\mathrm{H}(15 \mathrm{~B})$ & 109.5 \\
\hline $\mathrm{Si}(2)-\mathrm{C}(11)-\mathrm{H}(11 \mathrm{~A})$ & 109.5 & $\mathrm{H}(15 \mathrm{~A})-\mathrm{C}(15)-\mathrm{H}(15 \mathrm{~B})$ & 109.5 \\
\hline $\mathrm{Si}(2)-\mathrm{C}(11)-\mathrm{H}(11 \mathrm{~B})$ & 109.5 & $\mathrm{Si}(1)-\mathrm{C}(15)-\mathrm{H}(15 \mathrm{C})$ & 109.5 \\
\hline $\mathrm{H}(11 \mathrm{~A})-\mathrm{C}(11)-\mathrm{H}(11 \mathrm{~B})$ & 109.5 & $\mathrm{H}(15 \mathrm{~A})-\mathrm{C}(15)-\mathrm{H}(15 \mathrm{C})$ & 109.5 \\
\hline $\mathrm{Si}(2)-\mathrm{C}(11)-\mathrm{H}(11 \mathrm{C})$ & 109.5 & $\mathrm{H}(15 \mathrm{~B})-\mathrm{C}(15)-\mathrm{H}(15 \mathrm{C})$ & 109.5 \\
\hline
\end{tabular}


Stable 18. Crystal data and structure refinement for $4-C_{7} H_{8}$

Identification code

Empirical formula

Formula weight

Temperature

Wavelength

Crystal system

Space group

Unit cell dimensions

Volume

Z

Density (calculated)

Absorption coefficient

$\mathrm{F}(000)$

Crystal size

Theta range for data collection

Index ranges

Reflections collected

Independent reflections

Completeness to theta $=26.04^{\circ}$

Absorption correction

Refinement method

Data / restraints / parameters

Goodness-of-fit on $\mathrm{F}^{2}$ q317

C45H66N8Si2Lu2

1125.18

187(2) K

$0.71073 \AA$

Tetragonal

P4(3)2(1)2

$a=14.9571(7) \AA$

$\alpha=90^{\circ}$.

$\mathrm{b}=14.9571(7) \AA$

$\beta=90^{\circ}$.

$\mathrm{c}=21.8098(14) \AA$

$\gamma=90^{\circ}$

4879.2(4) $\AA^{3}$

4

$1.532 \mathrm{Mg} / \mathrm{m}^{3}$

$4.110 \mathrm{~mm}^{-1}$

2248

$0.128 \times 0.093 \times 0.074 \mathrm{~mm}^{3}$

1.65 to $26.04^{\circ}$.

$-18<=\mathrm{h}<=13,-18<=\mathrm{k}<=18,-26<=\mathrm{l}<=25$

27553

$4826[\mathrm{R}(\mathrm{int})=0.0578]$

$100.0 \%$

None

Full-matrix least-squares on $\mathrm{F}^{2}$

$4826 / 0 / 266$

0.965 
Final $\mathrm{R}$ indices [I $>2 \operatorname{sigma}(\mathrm{I})]$

$\mathrm{R}$ indices (all data)

Largest diff. peak and hole
$\mathrm{R} 1=0.0184, \mathrm{wR} 2=0.0406$

$\mathrm{R} 1=0.0205, \mathrm{wR} 2=0.0411$

0.738 and $-0.537 \mathrm{e}^{-3}$ 
Stable 19. Atomic coordinates ( $\times \mathbf{1 0}^{4}$ ) and equivalent isotropic displacement parameters $\left(\AA^{2} \times 10^{3}\right)$ for $4-C_{7} H_{8}$. U(eq) is defined as one third of the trace of the orthogonalized Uij tensor.

\begin{tabular}{|c|c|c|c|c|}
\hline & $\mathrm{x}$ & $\mathrm{y}$ & $\mathrm{z}$ & $\mathrm{U}(\mathrm{eq})$ \\
\hline $\mathrm{Lu}(1)$ & $10250(1)$ & $10250(1)$ & 0 & $24(1)$ \\
\hline $\mathrm{Lu}(2)$ & $8275(1)$ & $8275(1)$ & 0 & $24(1)$ \\
\hline $\operatorname{Si}(1)$ & $10696(1)$ & $12470(1)$ & $786(1)$ & $48(1)$ \\
\hline $\mathrm{N}(1)$ & $9779(2)$ & $8663(2)$ & $353(1)$ & $27(1)$ \\
\hline $\mathrm{N}(2)$ & $6749(2)$ & $8095(2)$ & $342(1)$ & $32(1)$ \\
\hline $\mathrm{N}(3)$ & $7923(2)$ & $9414(2)$ & $792(1)$ & $29(1)$ \\
\hline $\mathrm{N}(4)$ & $8521(2)$ & $7265(2)$ & $832(1)$ & $34(1)$ \\
\hline $\mathrm{C}(1)$ & $10194(2)$ & $8997(2)$ & $858(2)$ & $33(1)$ \\
\hline$C(2)$ & $11112(2)$ & $9063(2)$ & $766(2)$ & $40(1)$ \\
\hline$C(3)$ & $11279(2)$ & $8748(2)$ & $172(2)$ & $38(1)$ \\
\hline$C(4)$ & $10450(2)$ & $8508(2)$ & $-65(2)$ & $30(1)$ \\
\hline$C(5)$ & $8117(2)$ & $10224(2)$ & $660(2)$ & $32(1)$ \\
\hline$C(6)$ & $7461(2)$ & $9254(2)$ & $1382(2)$ & $36(1)$ \\
\hline$C(7)$ & $6495(2)$ & $8935(3)$ & $1311(2)$ & $39(1)$ \\
\hline$C(8)$ & $5936(2)$ & $9643(2)$ & $991(2)$ & $45(1)$ \\
\hline$C(9)$ & $6121(3)$ & $8785(3)$ & $1958(2)$ & $57(1)$ \\
\hline$C(10)$ & $6448(2)$ & $8032(2)$ & $975(2)$ & $38(1)$ \\
\hline $\mathrm{C}(11)$ & $6152(2)$ & $8146(2)$ & $-77(2)$ & $37(1)$ \\
\hline$C(12)$ & $8735(2)$ & $7272(3)$ & $1432(2)$ & $38(1)$ \\
\hline$C(13)$ & $8673(2)$ & $6421(3)$ & $1690(2)$ & $45(1)$ \\
\hline
\end{tabular}




\begin{tabular}{lrrrr}
$\mathrm{C}(14)$ & $8420(2)$ & $5854(3)$ & $1220(2)$ & $44(1)$ \\
$\mathrm{C}(15)$ & $8328(2)$ & $6386(2)$ & $697(2)$ & $36(1)$ \\
$\mathrm{C}(16)$ & $10593(3)$ & $11247(2)$ & $776(2)$ & $44(1)$ \\
$\mathrm{C}(17)$ & $10930(3)$ & $12957(3)$ & $17(2)$ & $75(1)$ \\
$\mathrm{C}(18)$ & $11654(3)$ & $12819(3)$ & $1288(2)$ & $79(2)$ \\
$\mathrm{C}(19)$ & $9642(3)$ & $13016(3)$ & $1081(2)$ & $85(2)$ \\
$\mathrm{C}(20)$ & $10736(3)$ & $-736(3)$ & 2500 & $53(1)$ \\
$\mathrm{C}(21)$ & $10959(3)$ & $151(3)$ & $2387(2)$ & $56(1)$ \\
$\mathrm{C}(22)$ & $10305(3)$ & $793(3)$ & $2397(2)$ & $57(1)$ \\
$\mathrm{C}(23)$ & $9426(3)$ & $574(3)$ & 2500 & $59(2)$ \\
$\mathrm{C}(24)$ & $8710(3)$ & $1290(3)$ & 2500 & $89(2)$ \\
\hline
\end{tabular}


STable 20. Anisotropic displacement parameters $\left(\AA^{2} \times 1^{3}\right)$ for $4-C_{7} H_{8}$. The anisotropic displacement factor exponent takes the form: $-2 \pi^{2}\left[h^{2} a * 2 U^{11}+\ldots\right.$ $\left.+2 \mathrm{~h} \mathrm{k} \mathrm{a*} \mathbf{b}^{*} \mathbf{U}^{12}\right]$

\begin{tabular}{ccccccc}
\hline & $\mathrm{U} 11$ & $\mathrm{U} 22$ & $\mathrm{U} 33$ & $\mathrm{U} 23$ & $\mathrm{U} 13$ & $\mathrm{U} 12$ \\
& & & & & & \\
\hline $\mathrm{Lu}(1)$ & $22(1)$ & $22(1)$ & $29(1)$ & $2(1)$ & $-2(1)$ & $-4(1)$ \\
$\mathrm{Lu}(2)$ & $20(1)$ & $20(1)$ & $32(1)$ & $0(1)$ & $0(1)$ & $-2(1)$ \\
$\mathrm{Si}(1)$ & $45(1)$ & $32(1)$ & $66(1)$ & $-11(1)$ & $-4(1)$ & $-6(1)$ \\
$\mathrm{N}(1)$ & $27(1)$ & $20(1)$ & $34(1)$ & $6(1)$ & $-1(1)$ & $-3(1)$ \\
$\mathrm{N}(2)$ & $29(2)$ & $22(2)$ & $46(2)$ & $-3(1)$ & $5(1)$ & $-2(1)$ \\
$\mathrm{N}(3)$ & $25(2)$ & $28(2)$ & $34(1)$ & $0(1)$ & $1(1)$ & $-2(1)$ \\
$\mathrm{N}(4)$ & $26(2)$ & $31(2)$ & $45(2)$ & $6(1)$ & $2(1)$ & $-1(1)$ \\
$\mathrm{C}(1)$ & $38(2)$ & $28(2)$ & $31(2)$ & $8(1)$ & $-9(2)$ & $-8(2)$ \\
$\mathrm{C}(2)$ & $36(2)$ & $30(2)$ & $53(2)$ & $13(2)$ & $-16(2)$ & $-3(2)$ \\
$\mathrm{C}(3)$ & $19(2)$ & $30(2)$ & $64(2)$ & $9(2)$ & $-4(2)$ & $4(1)$ \\
$\mathrm{C}(4)$ & $24(2)$ & $20(2)$ & $45(2)$ & $3(2)$ & $-1(2)$ & $1(1)$ \\
$\mathrm{C}(5)$ & $22(2)$ & $29(2)$ & $44(2)$ & $-6(2)$ & $0(2)$ & $-1(1)$ \\
$\mathrm{C}(6)$ & $36(2)$ & $37(2)$ & $34(2)$ & $-3(2)$ & $6(2)$ & $0(2)$ \\
$\mathrm{C}(7)$ & $34(2)$ & $36(2)$ & $46(2)$ & $2(2)$ & $14(2)$ & $-3(2)$ \\
$\mathrm{C}(8)$ & $26(2)$ & $35(2)$ & $74(3)$ & $-6(2)$ & $9(2)$ & $-1(2)$ \\
$\mathrm{C}(9)$ & $60(3)$ & $53(3)$ & $57(2)$ & $2(2)$ & $27(2)$ & $-8(2)$ \\
$\mathrm{C}(10)$ & $27(2)$ & $30(2)$ & $56(2)$ & $3(2)$ & $11(2)$ & $-5(1)$ \\
$\mathrm{C}(11)$ & $20(2)$ & $25(2)$ & $66(3)$ & $-9(2)$ & $-1(2)$ & $-2(1)$ \\
$\mathrm{C}(12)$ & $28(2)$ & $42(2)$ & $45(2)$ & $9(2)$ & $2(2)$ & $3(2)$ \\
& $26(2)$ & $53(3)$ & $54(2)$ & $25(2)$ & $5(2)$ & $6(2)$
\end{tabular}




\begin{tabular}{rrrrrrr}
$\mathrm{C}(14)$ & $31(2)$ & $34(2)$ & $68(3)$ & $19(2)$ & $9(2)$ & $2(2)$ \\
$\mathrm{C}(15)$ & $19(2)$ & $30(2)$ & $59(2)$ & $8(2)$ & $10(2)$ & $2(2)$ \\
$\mathrm{C}(16)$ & $60(3)$ & $37(2)$ & $36(2)$ & $-1(2)$ & $-6(2)$ & $-7(2)$ \\
$\mathrm{C}(17)$ & $79(3)$ & $45(2)$ & $100(4)$ & $28(3)$ & $6(4)$ & $-3(2)$ \\
$\mathrm{C}(18)$ & $70(4)$ & $56(3)$ & $111(4)$ & $-34(3)$ & $-28(3)$ & $-5(3)$ \\
$\mathrm{C}(19)$ & $59(3)$ & $75(4)$ & $121(4)$ & $-45(3)$ & $4(3)$ & $-2(3)$ \\
$\mathrm{C}(20)$ & $59(2)$ & $59(2)$ & $42(3)$ & $-7(3)$ & $-7(3)$ & $18(3)$ \\
$\mathrm{C}(21)$ & $54(3)$ & $71(3)$ & $43(3)$ & $-9(2)$ & $0(2)$ & $2(2)$ \\
$\mathrm{C}(22)$ & $78(3)$ & $50(2)$ & $42(3)$ & $-5(2)$ & $-9(2)$ & $1(2)$ \\
$\mathrm{C}(23)$ & $66(2)$ & $66(2)$ & $44(3)$ & $-13(3)$ & $-13(3)$ & $17(3)$ \\
$\mathrm{C}(24)$ & $85(3)$ & & & & & \\
\hline
\end{tabular}


Stable 21. Bond lengths $[\AA ̊]$ and angles $\left[{ }^{\circ}\right]$ for $4-\mathrm{C}_{7} \mathrm{H}_{8}$

\begin{tabular}{|c|c|c|c|}
\hline $\mathrm{Lu}(1)-\mathrm{C}(16) \# 1$ & $2.312(4)$ & $\mathrm{N}(1)-\mathrm{C}(4)$ & $1.376(4)$ \\
\hline $\mathrm{Lu}(1)-\mathrm{C}(16)$ & $2.312(4)$ & $\mathrm{N}(2)-\mathrm{C}(11)$ & $1.280(4)$ \\
\hline $\mathrm{Lu}(1)-\mathrm{N}(1) \# 1$ & $2.593(2)$ & $\mathrm{N}(2)-\mathrm{C}(10)$ & $1.456(4)$ \\
\hline $\mathrm{Lu}(1)-\mathrm{N}(1)$ & $2.593(2)$ & $\mathrm{N}(3)-\mathrm{C}(5)$ & $1.279(4)$ \\
\hline $\mathrm{Lu}(1)-\mathrm{C}(4)$ & $2.627(3)$ & $\mathrm{N}(3)-\mathrm{C}(6)$ & $1.479(4)$ \\
\hline $\mathrm{Lu}(1)-\mathrm{C}(4) \# 1$ & $2.627(3)$ & $\mathrm{N}(4)-\mathrm{C}(12)$ & $1.349(4)$ \\
\hline $\mathrm{Lu}(1)-\mathrm{C}(1) \# 1$ & $2.650(3)$ & $\mathrm{N}(4)-\mathrm{C}(15)$ & $1.377(4)$ \\
\hline $\mathrm{Lu}(1)-\mathrm{C}(1)$ & $2.650(3)$ & $C(1)-C(2)$ & $1.391(5)$ \\
\hline $\mathrm{Lu}(1)-\mathrm{C}(3)$ & $2.749(3)$ & $\mathrm{C}(1)-\mathrm{H}(1)$ & 0.9300 \\
\hline $\mathrm{Lu}(1)-\mathrm{C}(3) \# 1$ & $2.749(3)$ & $C(2)-C(3)$ & $1.400(5)$ \\
\hline $\mathrm{Lu}(1)-\mathrm{C}(2) \# 1$ & $2.759(3)$ & $\mathrm{C}(2)-\mathrm{H}(2)$ & 0.9300 \\
\hline $\mathrm{Lu}(1)-\mathrm{C}(2)$ & $2.759(3)$ & $C(3)-C(4)$ & $1.391(5)$ \\
\hline $\mathrm{Lu}(2)-\mathrm{N}(4) \# 1$ & $2.389(3)$ & $\mathrm{C}(3)-\mathrm{H}(3)$ & 0.9300 \\
\hline $\mathrm{Lu}(2)-\mathrm{N}(4)$ & $2.389(3)$ & $C(4)-C(5) \# 1$ & $1.462(5)$ \\
\hline $\mathrm{Lu}(2)-\mathrm{N}(2) \# 1$ & $2.416(3)$ & $C(5)-C(4) \# 1$ & $1.462(5)$ \\
\hline $\mathrm{Lu}(2)-\mathrm{N}(2)$ & $2.416(3)$ & $\mathrm{C}(5)-\mathrm{H}(5)$ & 0.9300 \\
\hline $\mathrm{Lu}(2)-\mathrm{N}(1)$ & $2.448(3)$ & $C(6)-C(7)$ & $1.529(5)$ \\
\hline $\mathrm{Lu}(2)-\mathrm{N}(1) \# 1$ & $2.448(3)$ & $\mathrm{C}(6)-\mathrm{H}(6 \mathrm{~A})$ & 0.9700 \\
\hline $\mathrm{Lu}(2)-\mathrm{N}(3) \# 1$ & $2.483(3)$ & $\mathrm{C}(6)-\mathrm{H}(6 \mathrm{~B})$ & 0.9700 \\
\hline $\mathrm{Lu}(2)-\mathrm{N}(3)$ & $2.483(3)$ & $\mathrm{C}(7)-\mathrm{C}(8)$ & $1.519(5)$ \\
\hline $\operatorname{Si}(1)-C(16)$ & $1.836(4)$ & $\mathrm{C}(7)-\mathrm{C}(9)$ & $1.533(5)$ \\
\hline $\operatorname{Si}(1)-C(17)$ & $1.861(4)$ & $C(7)-C(10)$ & $1.538(5)$ \\
\hline $\operatorname{Si}(1)-C(18)$ & $1.878(4)$ & $\mathrm{C}(8)-\mathrm{H}(8 \mathrm{~A})$ & 0.9600 \\
\hline $\mathrm{Si}(1)-\mathrm{C}(19)$ & $1.887(5)$ & $\mathrm{C}(8)-\mathrm{H}(8 \mathrm{~B})$ & 0.9600 \\
\hline $\mathrm{N}(1)-\mathrm{C}(1)$ & $1.360(4)$ & $\mathrm{C}(8)-\mathrm{H}(8 \mathrm{C})$ & 0.9600 \\
\hline
\end{tabular}




\begin{tabular}{|c|c|c|c|}
\hline $\mathrm{C}(9)-\mathrm{H}(9 \mathrm{~A})$ & 0.9600 & $\mathrm{C}(18)-\mathrm{H}(18 \mathrm{~A})$ & 0.9600 \\
\hline $\mathrm{C}(9)-\mathrm{H}(9 \mathrm{~B})$ & 0.9600 & $\mathrm{C}(18)-\mathrm{H}(18 \mathrm{~B})$ & 0.9600 \\
\hline $\mathrm{C}(9)-\mathrm{H}(9 \mathrm{C})$ & 0.9600 & $\mathrm{C}(18)-\mathrm{H}(18 \mathrm{C})$ & 0.9600 \\
\hline $\mathrm{C}(10)-\mathrm{H}(10 \mathrm{~A})$ & 0.9700 & $\mathrm{C}(19)-\mathrm{H}(19 \mathrm{~A})$ & 0.9600 \\
\hline $\mathrm{C}(10)-\mathrm{H}(10 \mathrm{~B})$ & 0.9700 & $\mathrm{C}(19)-\mathrm{H}(19 \mathrm{~B})$ & 0.9600 \\
\hline $\mathrm{C}(11)-\mathrm{C}(15) \# 1$ & $1.423(5)$ & $\mathrm{C}(19)-\mathrm{H}(19 \mathrm{C})$ & 0.9600 \\
\hline $\mathrm{C}(11)-\mathrm{H}(11)$ & 0.9300 & $C(20)-C(21)$ & $1.390(5)$ \\
\hline$C(12)-C(13)$ & $1.394(5)$ & $\mathrm{C}(20)-\mathrm{C}(21) \# 2$ & $1.390(5)$ \\
\hline $\mathrm{C}(12)-\mathrm{H}(12)$ & 0.9300 & $\mathrm{C}(20)-\mathrm{H}(20)$ & 0.9300 \\
\hline$C(13)-C(14)$ & $1.382(6)$ & $\mathrm{C}(21)-\mathrm{C}(22)$ & $1.371(5)$ \\
\hline $\mathrm{C}(13)-\mathrm{H}(13)$ & 0.9300 & $\mathrm{C}(21)-\mathrm{H}(21)$ & 0.9300 \\
\hline$C(14)-C(15)$ & $1.398(5)$ & $C(22)-C(23)$ & $1.374(5)$ \\
\hline $\mathrm{C}(14)-\mathrm{H}(14)$ & 0.9300 & $\mathrm{C}(22)-\mathrm{H}(22)$ & 0.9300 \\
\hline$C(15)-C(11) \# 1$ & $1.423(5)$ & $\mathrm{C}(23)-\mathrm{C}(22) \# 2$ & $1.374(5)$ \\
\hline $\mathrm{C}(16)-\mathrm{H}(16 \mathrm{~A})$ & 0.9700 & $C(23)-C(24)$ & $1.514(8)$ \\
\hline $\mathrm{C}(16)-\mathrm{H}(16 \mathrm{~B})$ & 0.9700 & $\mathrm{C}(24)-\mathrm{H}(24 \mathrm{~A})$ & 0.9600 \\
\hline $\mathrm{C}(17)-\mathrm{H}(17 \mathrm{~A})$ & 0.9600 & $\mathrm{C}(24)-\mathrm{H}(24 \mathrm{~B})$ & 0.9600 \\
\hline $\mathrm{C}(17)-\mathrm{H}(17 \mathrm{~B})$ & 0.9600 & $\mathrm{C}(24)-\mathrm{H}(24 \mathrm{C})$ & 0.9600 \\
\hline $\mathrm{C}(17)-\mathrm{H}(17 \mathrm{C})$ & 0.9600 & & \\
\hline $\mathrm{C}(16) \# 1-\mathrm{Lu}(1)-\mathrm{C}(16)$ & $104.47(19)$ & $\mathrm{N}(1) \# 1-\mathrm{Lu}(1)-\mathrm{C}(4)$ & $79.54(9)$ \\
\hline $\mathrm{C}(16) \# 1-\mathrm{Lu}(1)-\mathrm{N}(1) \# 1$ & $115.66(11)$ & $\mathrm{N}(1)-\mathrm{Lu}(1)-\mathrm{C}(4)$ & $30.56(9)$ \\
\hline $\mathrm{C}(16)-\mathrm{Lu}(1)-\mathrm{N}(1) \# 1$ & $126.53(12)$ & $\mathrm{C}(16) \# 1-\mathrm{Lu}(1)-\mathrm{C}(4) \# 1$ & $130.83(12)$ \\
\hline $\mathrm{C}(16) \# 1-\mathrm{Lu}(1)-\mathrm{N}(1)$ & $126.53(12)$ & $\mathrm{C}(16)-\mathrm{Lu}(1)-\mathrm{C}(4) \# 1$ & $96.13(13)$ \\
\hline $\mathrm{C}(16)-\mathrm{Lu}(1)-\mathrm{N}(1)$ & $115.66(11)$ & $\mathrm{N}(1) \# 1-\mathrm{Lu}(1)-\mathrm{C}(4) \# 1$ & $30.56(9)$ \\
\hline $\mathrm{N}(1) \# 1-\mathrm{Lu}(1)-\mathrm{N}(1)$ & $65.81(11)$ & $\mathrm{N}(1)-\mathrm{Lu}(1)-\mathrm{C}(4) \# 1$ & $79.54(9)$ \\
\hline $\mathrm{C}(16) \# 1-\mathrm{Lu}(1)-\mathrm{C}(4)$ & $96.13(13)$ & $C(4)-L u(1)-C(4) \# 1$ & $103.23(13)$ \\
\hline $\mathrm{C}(16)-\mathrm{Lu}(1)-\mathrm{C}(4)$ & $130.83(12)$ & $\mathrm{C}(16) \# 1-\mathrm{Lu}(1)-\mathrm{C}(1) \# 1$ & $86.91(12)$ \\
\hline
\end{tabular}




\begin{tabular}{|c|c|c|c|}
\hline$C(16)-\mathrm{Lu}(1)-\mathrm{C}(1) \# 1$ & $133.95(13)$ & $\mathrm{C}(1)-\mathrm{Lu}(1)-\mathrm{C}(3) \# 1$ & $117.81(11)$ \\
\hline $\mathrm{N}(1) \# 1-\mathrm{Lu}(1)-\mathrm{C}(1) \# 1$ & $30.04(8)$ & $\mathrm{C}(3)-\mathrm{Lu}(1)-\mathrm{C}(3) \# 1$ & $159.04(16)$ \\
\hline $\mathrm{N}(1)-\mathrm{Lu}(1)-\mathrm{C}(1) \# 1$ & $89.32(9)$ & $\mathrm{C}(16) \# 1-\mathrm{Lu}(1)-\mathrm{C}(2) \# 1$ & $82.43(13)$ \\
\hline $\mathrm{C}(4)-\mathrm{Lu}(1)-\mathrm{C}(1) \# 1$ & $90.62(11)$ & $\mathrm{C}(16)-\mathrm{Lu}(1)-\mathrm{C}(2) \# 1$ & $106.55(13)$ \\
\hline $\mathrm{C}(4) \# 1-\mathrm{Lu}(1)-\mathrm{C}(1) \# 1$ & $48.72(11)$ & $\mathrm{N}(1) \# 1-\mathrm{Lu}(1)-\mathrm{C}(2) \# 1$ & $50.04(10)$ \\
\hline $\mathrm{C}(16) \# 1-\mathrm{Lu}(1)-\mathrm{C}(1)$ & $133.95(13)$ & $\mathrm{N}(1)-\mathrm{Lu}(1)-\mathrm{C}(2) \# 1$ & $115.62(10)$ \\
\hline $\mathrm{C}(16)-\mathrm{Lu}(1)-\mathrm{C}(1)$ & $86.91(12)$ & $\mathrm{C}(4)-\mathrm{Lu}(1)-\mathrm{C}(2) \# 1$ & $120.27(11)$ \\
\hline $\mathrm{N}(1) \# 1-\mathrm{Lu}(1)-\mathrm{C}(1)$ & $89.32(9)$ & $\mathrm{C}(4) \# 1-\mathrm{Lu}(1)-\mathrm{C}(2) \# 1$ & $48.76(11)$ \\
\hline $\mathrm{N}(1)-\mathrm{Lu}(1)-\mathrm{C}(1)$ & $30.04(8)$ & $\mathrm{C}(1) \# 1-\mathrm{Lu}(1)-\mathrm{C}(2) \# 1$ & $29.73(11)$ \\
\hline $\mathrm{C}(4)-\mathrm{Lu}(1)-\mathrm{C}(1)$ & $48.72(11)$ & $\mathrm{C}(1)-\mathrm{Lu}(1)-\mathrm{C}(2) \# 1$ & $137.54(11)$ \\
\hline $\mathrm{C}(4) \# 1-\mathrm{Lu}(1)-\mathrm{C}(1)$ & $90.62(11)$ & $\mathrm{C}(3)-\mathrm{Lu}(1)-\mathrm{C}(2) \# 1$ & $145.61(10)$ \\
\hline $\mathrm{C}(1) \# 1-\mathrm{Lu}(1)-\mathrm{C}(1)$ & $116.98(14)$ & $\mathrm{C}(3) \# 1-\mathrm{Lu}(1)-\mathrm{C}(2) \# 1$ & $29.46(10)$ \\
\hline $\mathrm{C}(16) \# 1-\mathrm{Lu}(1)-\mathrm{C}(3)$ & $85.42(12)$ & $\mathrm{C}(16) \# 1-\mathrm{Lu}(1)-\mathrm{C}(2)$ & $106.55(13)$ \\
\hline $\mathrm{C}(16)-\mathrm{Lu}(1)-\mathrm{C}(3)$ & $107.62(13)$ & $\mathrm{C}(16)-\mathrm{Lu}(1)-\mathrm{C}(2)$ & $82.43(13)$ \\
\hline $\mathrm{N}(1) \# 1-\mathrm{Lu}(1)-\mathrm{C}(3)$ & $109.32(10)$ & $\mathrm{N}(1) \# 1-\mathrm{Lu}(1)-\mathrm{C}(2)$ & $115.62(10)$ \\
\hline $\mathrm{N}(1)-\mathrm{Lu}(1)-\mathrm{C}(3)$ & $50.48(10)$ & $\mathrm{N}(1)-\mathrm{Lu}(1)-\mathrm{C}(2)$ & $50.04(10)$ \\
\hline $\mathrm{C}(4)-\mathrm{Lu}(1)-\mathrm{C}(3)$ & $29.88(10)$ & $\mathrm{C}(4)-\mathrm{Lu}(1)-\mathrm{C}(2)$ & $48.76(11)$ \\
\hline $\mathrm{C}(4) \# 1-\mathrm{Lu}(1)-\mathrm{C}(3)$ & $129.88(11)$ & $\mathrm{C}(4) \# 1-\mathrm{Lu}(1)-\mathrm{C}(2)$ & $120.27(11)$ \\
\hline $\mathrm{C}(1) \# 1-\mathrm{Lu}(1)-\mathrm{C}(3)$ & $117.81(11)$ & $\mathrm{C}(1) \# 1-\mathrm{Lu}(1)-\mathrm{C}(2)$ & $137.54(11)$ \\
\hline $\mathrm{C}(1)-\mathrm{Lu}(1)-\mathrm{C}(3)$ & $48.95(11)$ & $\mathrm{C}(1)-\mathrm{Lu}(1)-\mathrm{C}(2)$ & $29.73(11)$ \\
\hline $\mathrm{C}(16) \# 1-\mathrm{Lu}(1)-\mathrm{C}(3) \# 1$ & $107.62(13)$ & $\mathrm{C}(3)-\mathrm{Lu}(1)-\mathrm{C}(2)$ & $29.46(10)$ \\
\hline$C(16)-\mathrm{Lu}(1)-\mathrm{C}(3) \# 1$ & $85.42(12)$ & $\mathrm{C}(3) \# 1-\mathrm{Lu}(1)-\mathrm{C}(2)$ & $145.61(10)$ \\
\hline $\mathrm{N}(1) \# 1-\mathrm{Lu}(1)-\mathrm{C}(3) \# 1$ & $50.48(10)$ & $\mathrm{C}(2) \# 1-\mathrm{Lu}(1)-\mathrm{C}(2)$ & $165.64(16)$ \\
\hline $\mathrm{N}(1)-\mathrm{Lu}(1)-\mathrm{C}(3) \# 1$ & $109.32(10)$ & $\mathrm{N}(4) \# 1-\mathrm{Lu}(2)-\mathrm{N}(4)$ & $140.46(13)$ \\
\hline $\mathrm{C}(4)-\mathrm{Lu}(1)-\mathrm{C}(3) \# 1$ & $129.88(11)$ & $\mathrm{N}(4) \# 1-\mathrm{Lu}(2)-\mathrm{N}(2) \# 1$ & $80.84(9)$ \\
\hline $\mathrm{C}(4) \# 1-\mathrm{Lu}(1)-\mathrm{C}(3) \# 1$ & $29.88(10)$ & $\mathrm{N}(4)-\mathrm{Lu}(2)-\mathrm{N}(2) \# 1$ & $69.77(10)$ \\
\hline $\mathrm{C}(1) \# 1-\mathrm{Lu}(1)-\mathrm{C}(3) \# 1$ & $48.95(11)$ & $\mathrm{N}(4) \# 1-\mathrm{Lu}(2)-\mathrm{N}(2)$ & $69.77(10)$ \\
\hline
\end{tabular}




\begin{tabular}{|c|c|c|c|}
\hline $\mathrm{N}(4)-\mathrm{Lu}(2)-\mathrm{N}(2)$ & $80.84(9)$ & $\mathrm{C}(16)-\mathrm{Si}(1)-\mathrm{C}(19)$ & $111.4(2)$ \\
\hline $\mathrm{N}(2) \# 1-\mathrm{Lu}(2)-\mathrm{N}(2)$ & $83.42(12)$ & $\mathrm{C}(17)-\mathrm{Si}(1)-\mathrm{C}(19)$ & $107.2(2)$ \\
\hline $\mathrm{N}(4) \# 1-\mathrm{Lu}(2)-\mathrm{N}(1)$ & $141.57(9)$ & $\mathrm{C}(18)-\mathrm{Si}(1)-\mathrm{C}(19)$ & $108.5(2)$ \\
\hline $\mathrm{N}(4)-\mathrm{Lu}(2)-\mathrm{N}(1)$ & $76.70(9)$ & $\mathrm{C}(1)-\mathrm{N}(1)-\mathrm{C}(4)$ & $105.4(3)$ \\
\hline $\mathrm{N}(2) \# 1-\mathrm{Lu}(2)-\mathrm{N}(1)$ & $115.06(9)$ & $\mathrm{C}(1)-\mathrm{N}(1)-\mathrm{Lu}(2)$ & $139.6(2)$ \\
\hline $\mathrm{N}(2)-\mathrm{Lu}(2)-\mathrm{N}(1)$ & $142.90(9)$ & $\mathrm{C}(4)-\mathrm{N}(1)-\mathrm{Lu}(2)$ & $114.98(19)$ \\
\hline $\mathrm{N}(4) \# 1-\mathrm{Lu}(2)-\mathrm{N}(1) \# 1$ & $76.70(8)$ & $\mathrm{C}(1)-\mathrm{N}(1)-\mathrm{Lu}(1)$ & $77.29(17)$ \\
\hline $\mathrm{N}(4)-\mathrm{Lu}(2)-\mathrm{N}(1) \# 1$ & $141.57(9)$ & $\mathrm{C}(4)-\mathrm{N}(1)-\mathrm{Lu}(1)$ & $76.08(16)$ \\
\hline $\mathrm{N}(2) \# 1-\mathrm{Lu}(2)-\mathrm{N}(1) \# 1$ & $142.90(9)$ & $\mathrm{Lu}(2)-\mathrm{N}(1)-\mathrm{Lu}(1)$ & $111.96(9)$ \\
\hline $\mathrm{N}(2)-\mathrm{Lu}(2)-\mathrm{N}(1) \# 1$ & $115.06(9)$ & $\mathrm{C}(11)-\mathrm{N}(2)-\mathrm{C}(10)$ & $117.8(3)$ \\
\hline $\mathrm{N}(1)-\mathrm{Lu}(2)-\mathrm{N}(1) \# 1$ & $70.27(11)$ & $\mathrm{C}(11)-\mathrm{N}(2)-\mathrm{Lu}(2)$ & $115.6(2)$ \\
\hline $\mathrm{N}(4) \# 1-\mathrm{Lu}(2)-\mathrm{N}(3) \# 1$ & $86.46(10)$ & $\mathrm{C}(10)-\mathrm{N}(2)-\mathrm{Lu}(2)$ & $126.3(2)$ \\
\hline $\mathrm{N}(4)-\mathrm{Lu}(2)-\mathrm{N}(3) \# 1$ & $106.78(9)$ & $C(5)-N(3)-C(6)$ & $117.1(3)$ \\
\hline $\mathrm{N}(2) \# 1-\mathrm{Lu}(2)-\mathrm{N}(3) \# 1$ & $70.21(9)$ & $\mathrm{C}(5)-\mathrm{N}(3)-\mathrm{Lu}(2)$ & $116.4(2)$ \\
\hline$N(2)-\mathrm{Lu}(2)-\mathrm{N}(3) \# 1$ & $147.10(9)$ & $\mathrm{C}(6)-\mathrm{N}(3)-\mathrm{Lu}(2)$ & $126.4(2)$ \\
\hline $\mathrm{N}(1)-\mathrm{Lu}(2)-\mathrm{N}(3) \# 1$ & $68.79(8)$ & $\mathrm{C}(12)-\mathrm{N}(4)-\mathrm{C}(15)$ & $105.3(3)$ \\
\hline $\mathrm{N}(1) \# 1-\mathrm{Lu}(2)-\mathrm{N}(3) \# 1$ & $79.22(9)$ & $\mathrm{C}(12)-\mathrm{N}(4)-\mathrm{Lu}(2)$ & $140.3(2)$ \\
\hline $\mathrm{N}(4) \# 1-\mathrm{Lu}(2)-\mathrm{N}(3)$ & $106.78(9)$ & $\mathrm{C}(15)-\mathrm{N}(4)-\mathrm{Lu}(2)$ & $114.2(2)$ \\
\hline $\mathrm{N}(4)-\mathrm{Lu}(2)-\mathrm{N}(3)$ & $86.46(9)$ & $\mathrm{N}(1)-\mathrm{C}(1)-\mathrm{C}(2)$ & $111.1(3)$ \\
\hline $\mathrm{N}(2) \# 1-\mathrm{Lu}(2)-\mathrm{N}(3)$ & $147.10(9)$ & $\mathrm{N}(1)-\mathrm{C}(1)-\mathrm{Lu}(1)$ & $72.67(16)$ \\
\hline $\mathrm{N}(2)-\mathrm{Lu}(2)-\mathrm{N}(3)$ & $70.21(9)$ & $\mathrm{C}(2)-\mathrm{C}(1)-\mathrm{Lu}(1)$ & $79.48(19)$ \\
\hline $\mathrm{N}(1)-\mathrm{Lu}(2)-\mathrm{N}(3)$ & $79.22(9)$ & $\mathrm{N}(1)-\mathrm{C}(1)-\mathrm{H}(1)$ & 124.5 \\
\hline $\mathrm{N}(1) \# 1-\mathrm{Lu}(2)-\mathrm{N}(3)$ & $68.79(8)$ & $\mathrm{C}(2)-\mathrm{C}(1)-\mathrm{H}(1)$ & 124.5 \\
\hline $\mathrm{N}(3) \# 1-\mathrm{Lu}(2)-\mathrm{N}(3)$ & $140.79(12)$ & $\mathrm{Lu}(1)-\mathrm{C}(1)-\mathrm{H}(1)$ & 115.1 \\
\hline $\mathrm{C}(16)-\mathrm{Si}(1)-\mathrm{C}(17)$ & $113.28(19)$ & $C(1)-C(2)-C(3)$ & $106.6(3)$ \\
\hline $\mathrm{C}(16)-\mathrm{Si}(1)-\mathrm{C}(18)$ & $110.3(2)$ & $C(1)-C(2)-L u(1)$ & $70.79(17)$ \\
\hline $\mathrm{C}(17)-\mathrm{Si}(1)-\mathrm{C}(18)$ & $105.9(2)$ & $C(3)-C(2)-L u(1)$ & $74.88(18)$ \\
\hline
\end{tabular}




\begin{tabular}{|c|c|c|c|}
\hline $\mathrm{C}(1)-\mathrm{C}(2)-\mathrm{H}(2)$ & 126.7 & $C(8)-C(7)-C(10)$ & $111.6(3)$ \\
\hline $\mathrm{C}(3)-\mathrm{C}(2)-\mathrm{H}(2)$ & 126.7 & $C(6)-C(7)-C(10)$ & $111.4(3)$ \\
\hline $\mathrm{Lu}(1)-\mathrm{C}(2)-\mathrm{H}(2)$ & 119.6 & $C(9)-C(7)-C(10)$ & $107.0(3)$ \\
\hline$C(4)-C(3)-C(2)$ & $105.8(3)$ & $\mathrm{C}(7)-\mathrm{C}(8)-\mathrm{H}(8 \mathrm{~A})$ & 109.5 \\
\hline$C(4)-C(3)-\operatorname{Lu}(1)$ & $70.21(16)$ & $\mathrm{C}(7)-\mathrm{C}(8)-\mathrm{H}(8 \mathrm{~B})$ & 109.5 \\
\hline $\mathrm{C}(2)-\mathrm{C}(3)-\mathrm{Lu}(1)$ & $75.66(18)$ & $\mathrm{H}(8 \mathrm{~A})-\mathrm{C}(8)-\mathrm{H}(8 \mathrm{~B})$ & 109.5 \\
\hline $\mathrm{C}(4)-\mathrm{C}(3)-\mathrm{H}(3)$ & 127.1 & $\mathrm{C}(7)-\mathrm{C}(8)-\mathrm{H}(8 \mathrm{C})$ & 109.5 \\
\hline $\mathrm{C}(2)-\mathrm{C}(3)-\mathrm{H}(3)$ & 127.1 & $\mathrm{H}(8 \mathrm{~A})-\mathrm{C}(8)-\mathrm{H}(8 \mathrm{C})$ & 109.5 \\
\hline $\mathrm{Lu}(1)-\mathrm{C}(3)-\mathrm{H}(3)$ & 119.1 & $\mathrm{H}(8 \mathrm{~B})-\mathrm{C}(8)-\mathrm{H}(8 \mathrm{C})$ & 109.5 \\
\hline$N(1)-C(4)-C(3)$ & $111.1(3)$ & $\mathrm{C}(7)-\mathrm{C}(9)-\mathrm{H}(9 \mathrm{~A})$ & 109.5 \\
\hline$N(1)-C(4)-C(5) \# 1$ & $119.1(3)$ & $\mathrm{C}(7)-\mathrm{C}(9)-\mathrm{H}(9 \mathrm{~B})$ & 109.5 \\
\hline$C(3)-C(4)-C(5) \# 1$ & $129.8(3)$ & $\mathrm{H}(9 \mathrm{~A})-\mathrm{C}(9)-\mathrm{H}(9 \mathrm{~B})$ & 109.5 \\
\hline $\mathrm{N}(1)-\mathrm{C}(4)-\mathrm{Lu}(1)$ & $73.35(16)$ & $\mathrm{C}(7)-\mathrm{C}(9)-\mathrm{H}(9 \mathrm{C})$ & 109.5 \\
\hline$C(3)-C(4)-\operatorname{Lu}(1)$ & $79.91(18)$ & $\mathrm{H}(9 \mathrm{~A})-\mathrm{C}(9)-\mathrm{H}(9 \mathrm{C})$ & 109.5 \\
\hline $\mathrm{C}(5) \# 1-\mathrm{C}(4)-\mathrm{Lu}(1)$ & $114.7(2)$ & $\mathrm{H}(9 \mathrm{~B})-\mathrm{C}(9)-\mathrm{H}(9 \mathrm{C})$ & 109.5 \\
\hline$N(3)-C(5)-C(4) \# 1$ & $120.7(3)$ & $\mathrm{N}(2)-\mathrm{C}(10)-\mathrm{C}(7)$ & $112.4(3)$ \\
\hline $\mathrm{N}(3)-\mathrm{C}(5)-\mathrm{H}(5)$ & 119.7 & $\mathrm{~N}(2)-\mathrm{C}(10)-\mathrm{H}(10 \mathrm{~A})$ & 109.1 \\
\hline $\mathrm{C}(4) \# 1-\mathrm{C}(5)-\mathrm{H}(5)$ & 119.7 & $\mathrm{C}(7)-\mathrm{C}(10)-\mathrm{H}(10 \mathrm{~A})$ & 109.1 \\
\hline$N(3)-C(6)-C(7)$ & $113.9(3)$ & $\mathrm{N}(2)-\mathrm{C}(10)-\mathrm{H}(10 \mathrm{~B})$ & 109.1 \\
\hline $\mathrm{N}(3)-\mathrm{C}(6)-\mathrm{H}(6 \mathrm{~A})$ & 108.8 & $\mathrm{C}(7)-\mathrm{C}(10)-\mathrm{H}(10 \mathrm{~B})$ & 109.1 \\
\hline $\mathrm{C}(7)-\mathrm{C}(6)-\mathrm{H}(6 \mathrm{~A})$ & 108.8 & $\mathrm{H}(10 \mathrm{~A})-\mathrm{C}(10)-\mathrm{H}(10 \mathrm{~B})$ & 107.9 \\
\hline $\mathrm{N}(3)-\mathrm{C}(6)-\mathrm{H}(6 \mathrm{~B})$ & 108.8 & $\mathrm{~N}(2)-\mathrm{C}(11)-\mathrm{C}(15) \# 1$ & $121.2(3)$ \\
\hline $\mathrm{C}(7)-\mathrm{C}(6)-\mathrm{H}(6 \mathrm{~B})$ & 108.8 & $\mathrm{~N}(2)-\mathrm{C}(11)-\mathrm{H}\left(\begin{array}{ll}1 & 1\end{array}\right)$ & 119.4 \\
\hline $\mathrm{H}(6 \mathrm{~A})-\mathrm{C}(6)-\mathrm{H}(6 \mathrm{~B})$ & 107.7 & $\mathrm{C}(15) \# 1-\mathrm{C}(11)-\mathrm{H}(11)$ & 119.4 \\
\hline$C(8)-C(7)-C(6)$ & $110.4(3)$ & $\mathrm{N}(4)-\mathrm{C}(12)-\mathrm{C}(13)$ & $111.5(4)$ \\
\hline$C(8)-C(7)-C(9)$ & $108.9(3)$ & $\mathrm{N}(4)-\mathrm{C}(12)-\mathrm{H}(12)$ & 124.2 \\
\hline$C(6)-C(7)-C(9)$ & $107.4(3)$ & $\mathrm{C}(13)-\mathrm{C}(12)-\mathrm{H}(12)$ & 124.2 \\
\hline
\end{tabular}




\begin{tabular}{|c|c|c|c|}
\hline$C(14)-C(13)-C(12)$ & $106.3(3)$ & $\mathrm{Si}(1)-\mathrm{C}(19)-\mathrm{H}(19 \mathrm{~A})$ & 109.5 \\
\hline $\mathrm{C}(14)-\mathrm{C}(13)-\mathrm{H}(13)$ & 126.9 & $\mathrm{Si}(1)-\mathrm{C}(19)-\mathrm{H}(19 \mathrm{~B})$ & 109.5 \\
\hline $\mathrm{C}(12)-\mathrm{C}(13)-\mathrm{H}(13)$ & 126.9 & $H(19 A)-C(19)-H(19 B)$ & 109.5 \\
\hline$C(13)-C(14)-C(15)$ & $106.4(3)$ & $\mathrm{Si}(1)-\mathrm{C}(19)-\mathrm{H}(19 \mathrm{C})$ & 109.5 \\
\hline $\mathrm{C}(13)-\mathrm{C}(14)-\mathrm{H}(14)$ & 126.8 & $\mathrm{H}(19 \mathrm{~A})-\mathrm{C}(19)-\mathrm{H}(19 \mathrm{C})$ & 109.5 \\
\hline $\mathrm{C}(15)-\mathrm{C}(14)-\mathrm{H}(14)$ & 126.8 & $\mathrm{H}(19 \mathrm{~B})-\mathrm{C}(19)-\mathrm{H}(19 \mathrm{C})$ & 109.5 \\
\hline $\mathrm{N}(4)-\mathrm{C}(15)-\mathrm{C}(14)$ & $110.4(4)$ & $\mathrm{C}(21)-\mathrm{C}(20)-\mathrm{C}(21) \# 2$ & $119.3(5)$ \\
\hline $\mathrm{N}(4)-\mathrm{C}(15)-\mathrm{C}(11) \# 1$ & $118.5(3)$ & $\mathrm{C}(21)-\mathrm{C}(20)-\mathrm{H}(20)$ & 120.3 \\
\hline $\mathrm{C}(14)-\mathrm{C}(15)-\mathrm{C}(11) \# 1$ & $130.9(4)$ & $\mathrm{C}(21) \# 2-\mathrm{C}(20)-\mathrm{H}(20)$ & 120.3 \\
\hline $\mathrm{Si}(1)-\mathrm{C}(16)-\mathrm{Lu}(1)$ & $132.0(2)$ & $C(22)-C(21)-C(20)$ & $119.6(5)$ \\
\hline $\mathrm{Si}(1)-\mathrm{C}(16)-\mathrm{H}(16 \mathrm{~A})$ & 104.2 & $\mathrm{C}(22)-\mathrm{C}(21)-\mathrm{H}(21)$ & 120.2 \\
\hline $\mathrm{Lu}(1)-\mathrm{C}(16)-\mathrm{H}(16 \mathrm{~A})$ & 104.2 & $\mathrm{C}(20)-\mathrm{C}(21)-\mathrm{H}(21)$ & 120.2 \\
\hline $\mathrm{Si}(1)-\mathrm{C}(16)-\mathrm{H}(16 \mathrm{~B})$ & 104.2 & $C(21)-C(22)-C(23)$ & $121.3(5)$ \\
\hline $\mathrm{Lu}(1)-\mathrm{C}(16)-\mathrm{H}(16 \mathrm{~B})$ & 104.2 & $\mathrm{C}(21)-\mathrm{C}(22)-\mathrm{H}(22)$ & 119.3 \\
\hline $\mathrm{H}(16 \mathrm{~A})-\mathrm{C}(16)-\mathrm{H}(16 \mathrm{~B})$ & 105.5 & $\mathrm{C}(23)-\mathrm{C}(22)-\mathrm{H}(22)$ & 119.3 \\
\hline $\mathrm{Si}(1)-\mathrm{C}(17)-\mathrm{H}(17 \mathrm{~A})$ & 109.5 & $C(22)-C(23)-C(22) \# 2$ & $118.8(5)$ \\
\hline $\mathrm{Si}(1)-\mathrm{C}(17)-\mathrm{H}(17 \mathrm{~B})$ & 109.5 & $C(22)-C(23)-C(24)$ & $120.6(3)$ \\
\hline $\mathrm{H}(17 \mathrm{~A})-\mathrm{C}(17)-\mathrm{H}(17 \mathrm{~B})$ & 109.5 & $\mathrm{C}(22) \# 2-\mathrm{C}(23)-\mathrm{C}(24)$ & $120.6(3)$ \\
\hline $\mathrm{Si}(1)-\mathrm{C}(17)-\mathrm{H}(17 \mathrm{C})$ & 109.5 & $\mathrm{C}(23)-\mathrm{C}(24)-\mathrm{H}(24 \mathrm{~A})$ & 109.5 \\
\hline $\mathrm{H}(17 \mathrm{~A})-\mathrm{C}(17)-\mathrm{H}(17 \mathrm{C})$ & 109.5 & $\mathrm{C}(23)-\mathrm{C}(24)-\mathrm{H}(24 \mathrm{~B})$ & 109.5 \\
\hline $\mathrm{H}(17 \mathrm{~B})-\mathrm{C}(17)-\mathrm{H}(17 \mathrm{C})$ & 109.5 & $\mathrm{H}(24 \mathrm{~A})-\mathrm{C}(24)-\mathrm{H}(24 \mathrm{~B})$ & 109.5 \\
\hline $\mathrm{Si}(1)-\mathrm{C}(18)-\mathrm{H}(18 \mathrm{~A})$ & 109.5 & $\mathrm{C}(23)-\mathrm{C}(24)-\mathrm{H}(24 \mathrm{C})$ & 109.5 \\
\hline $\mathrm{Si}(1)-\mathrm{C}(18)-\mathrm{H}(18 \mathrm{~B})$ & 109.5 & $\mathrm{H}(24 \mathrm{~A})-\mathrm{C}(24)-\mathrm{H}(24 \mathrm{C})$ & 109.5 \\
\hline $\mathrm{H}(18 \mathrm{~A})-\mathrm{C}(18)-\mathrm{H}(18 \mathrm{~B})$ & 109.5 & $\mathrm{H}(24 \mathrm{~B})-\mathrm{C}(24)-\mathrm{H}(24 \mathrm{C})$ & 109.5 \\
\hline $\mathrm{Si}(1)-\mathrm{C}(18)-\mathrm{H}(18 \mathrm{C})$ & 109.5 & & \\
\hline $\mathrm{H}(18 \mathrm{~A})-\mathrm{C}(18)-\mathrm{H}(18 \mathrm{C})$ & 109.5 & \multicolumn{2}{|c|}{ \#---End of supporting information } \\
\hline $\mathrm{H}(18 \mathrm{~B})-\mathrm{C}(18)-\mathrm{H}(18 \mathrm{C})$ & 109.5 & & \\
\hline
\end{tabular}


